\title{
Methods of Monte Carlo Biasing Using Two-Dimensional Discrete Ordinates Adjoint Flux
}

\author{
J. S. Tang \\ P. N. Stevens \\ T. J. Hoffman
}




\section{DISCLAIMER}

This report was prepared as an account of work sponsored by an agency of the United States Government. Neither the United States Government nor any agency Thereof, nor any of their employees, makes any warranty, express or implied, or assumes any legal liability or responsibility for the accuracy, completeness, or usefulness of any information, apparatus, product, or process disclosed, or represents that its use would not infringe privately owned rights. Reference herein to any specific commercial product, process, or service by trade name, trademark, manufacturer, or otherwise does not necessarily constitute or imply its endorsement, recommendation, or favoring by the United States Government or any agency thereof. The views and opinions of authors expressed herein do not necessarily state or reflect those of the United States Government or any agency thereof. 


\section{DISCLAIMER}

Portions of this document may be illegible in electronic image products. Images are produced from the best available original document. 
Printed in the United States of America. Available from National Technical Information Service

U.S. Department of Commerce

5285 Port Royal Road, Springfield, Virginia 22161

Price: Printed Copy \$6.00; Microfiche \$2.25

This report was prepared as an account of work sponsored by the United States Government. Neither the United States nor the Energy Research and Development Administration/United States Nuclear Regulatory Commission, nor any of their employees, nor any of their contractors, subcontractors, or their employees, makes any warranty, express or implied, or assumes any legal liability or responsibility for the accuracy, completeness or usefulness of any information, apparatus, product or process disclosed, or represents that its use would not infringe privately owned rights. 


\title{
Methods of Monte Carlo Biasing Using Two-Dimensional Discrete Ordinates Adjoint Flux
}

\author{
J. S. Tang \\ P. N. Stevens \\ T. J. Hoffman
}

\section{OAK RIDGE NATIONAL. LABORATDRY}


Printed in the United States of America. Available from National Technical Information Service

U.S. Department of Commerce

5285 Port Royal Road, Springfield, Virginia 22161

Price: Printed Copy $\$ 6.00 ;$ Microfiche $\$ 2.25$

This report was prepared as an account of work sponsored by the United States Guvernment. Neither the United States nor the Energy Research and Development Administration/United States Nuclear Regulatory Commission, nor any of their employees, nor any of their contractors, subcontractors, or their employees, makes any warranty, express or implied, or assumes any legal liability or responsibility for the accuracy, completeness or usefulness of any information, apparatus, product or process disclosed, or represents that its use would not infringe privately owned rights. 
ORNL/TM-5414

UC-79d - LMFBR

Physics

\author{
Contract No. W-7405-eng-26 \\ Neutron Physics Division
}

METHODS OF MONTE CARLO BIASING USING

TWO-DIMENSIONAL DISCRETE ORDINATES ADJOINT FLUX*

J. S. Tang, ${ }^{\dagger}$ P. N. Stevens, ${ }^{+\dagger}$ and T. J. Hoffman ${ }^{+\dagger}$

JUNE 1976

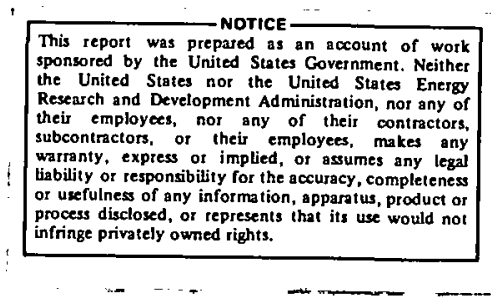

*Dissertation presented for the Doctor of Philosophy Degree at the University of Tennessee.

†Computer Sciences Division

${ }^{+}$Nuclear Engineering Department, University of Tennessee, Knoxville, TN.

\author{
NOTICE This document contains information of a preliminary nature \\ and was prepared primarily for internal use at the Oak Ridge National \\ Laboratory, !t is subiect to revision or renrrertinn and thargforo doos \\ not represent a final report. \\ OAK RIDGE NATIONAL LABORATORY \\ Oak Ridge, Tennessee 37830 \\ operated by \\ UNION CARIBDE CORPORATION \\ for the \\ ENERGY RESEARCH AND DEVELOPMENT ADMINISTRATION
}




\section{PREFACE AND ACKNOWLEDGEMENTS}

This work was performed by Jabo Sae Tang in partial fulfillment of the requirements for a doctoral degree in the Nuclear Engineering Department of the University of Tennessee. Special appreciation is extended to Ms. Juanita Rye who typed this manuscript. 
THIS PAGE

\section{WAS INTENTIONALLY LEFT BLANK}


Methods of biasing three-dimensional deep penetration Monte Carlo calculations using importance functions obtained from a twodimensional discrete ordinates adjoint calculation have been developed. The important distinction was made between the applications of the point value and the event value to alter the random walk in Monte Carlo analysis of radiation transport. The biasing techniques developed in this study are the angular probability biasing which alters the collision kernel using the point value as the importance function and the path length biasing which alters the transport kernel using the event value as the importance function. Source location biasings using the step importance function and the scalar adjoint flux obtained from the two-dimensional discrete ordinates adjoint calculation were also investigated.

The effects of the biasing techniques to Monte Carlo calculations have been investigated for neutron transport through a thick concrete shield with a penetrating duct. Source location biasing, angular probability biasing, and path length biasing were employed individually and in various combinations. Results of the biased Monte Carlo calculations were compared with the standard Monte Carlo and discrete ordinates calculations. Based upon the fractional standard deviations of the answers, the biasing techniques are a factor of 2 to 8 better than the standard method. Hence, the 
effectiveness and the applicability of the biasing techniques in deep penetration, three-dimensional Monte Carlo calculations are clearly demonstrated.

Results of Monte Carlo calculations using respectively the event value and the point value to alter the transport kernel showed that the event value calculation gave much better statistics (the FSD reduced by a factor of 2) than the point value calculation. Therefore, it was confirmed that the event value is the more appropiriate function for biasing the transport kernel. 
I. INTRODUCTION ......................... . . 1

II. THE ADJOINT BOLTZMANN TRANSPORT EQUATIONS . . . . . . . . 5

The Event Value and the Point Value Equations . . . . 5

Derivation of the Adjoint Boltzmann Equation from

the Value Functions.............. 9

Application of the Point Value and the Event Value

in Monte Carlo Biasings ............ . 13

II I. DEVELOPMENT OF MONTE CARLO IMPORTANCE SAMPLING

TECHNIQUES ........................ 14

Collision Mechanics . . . . . . . . . . . . 14

The Angular Probability Biasing Technique . . . . . . 22

The Path Length Biasing Technique . . . . . . . . 24

IV. DESCRIPTION OF THE STANDARD PROBLEM AND GENERATION OF.

IMPORTANCE FUNCTIONS . . . . . . . . . . . . 30

Description of the Standard Problem . . . . . . 30

Two-Dimensional Discrete Ordinates Adjoint

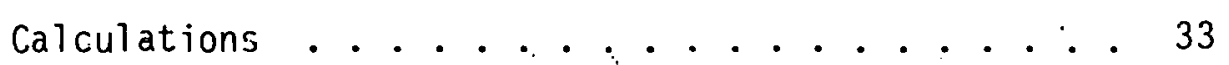

Determination of the Point Value . . . . . . . 34

Determination of the Event Value .......... 35

V. DESCRIPTION OF THE BIASING TECHNIQUES . . . . . . . . . . 44

Source Location Biasing . . . . . . . . . . . 44

Angular Probability Biasing . . . . . . . . . . 48 
Path Length Biasing . . . . . . . . . . 50

Russian Roulette and Splitting . . . . . . . 53

VI. DISCUSSION OF RESULTS . . . . . . . . . . . 55

Comparison of Results of Monte Carlo and Discrete

Ordinates Calculations .......... 57

Comparison of Fractional Standard Deviations . . . 67

Spatial Distribution of Collision Sites . . . . . 63

Path Length Biasing Using Different Importance

Functions ............... 70

Angular Probability Biasing Using Different Point

Values........................... 70

VII. CONCLUSIONS AND RECOMMENDATIONS . . . . . . . . . . 74

BIBLIOGRAPHY . . . . . . . . . . . . . . . . . 77 APPENDIXES

A. COMPUTER PROGRAMS ............... 81

B. COORDINATE TRANSFORMATIONS ............... 101

c. COMPARISON $0 \mathrm{~F} x^{*}$ and $w . . . . . . . . . . .106$

D. PLOTS OF ADJOINT DOT CALCULATIONS . . . . . . . . 109

E. NEUTRON CROSS SECTION FOR CONCRETE . . . . . . . 126 
LIST OF TABLES

TABLE

PAGE

1. Neutron Energy Group Structure . . . . . . . . . . . 32

2. Monte Carlo Calculations Performed ............ 56

3. Uncollided Neutron Flux of Monte Carlo Calculations of the Standard Problem . . . . . . . . . 58

4. Total Neutron Flux of Monte Carlo Calculations of the Standard Problem ............. . 59

5. Fractional Standard Deviations of the Total Neutron Flux from the Monte Carlo Calculations with Various Sampling Techniques for the Standard Problem . . . . . . 62

6. Comparison of Total Neutron Flux of Monte Carlo Calculations Using Different Importance Functions for Path Length Biasing . . . . . . . . . . . . 71

7. Comparison of Total Neutron Flux of Monte Carlo Calculations Using Different Point Values for Angular Probability Biasing . . . . . . . . . . . 72

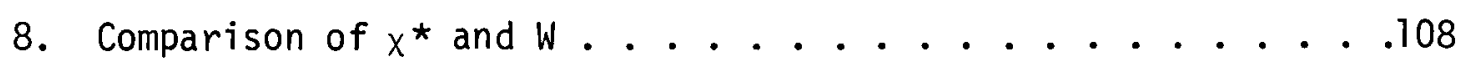

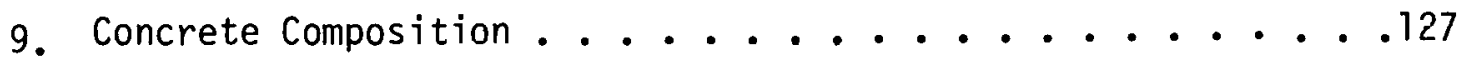




\section{LIST OF FIGURES}

FIGURE

PAGE

1. Coordinate System which Relates a Fixed Point $\bar{r}$ to an Arbitrary Point $\bar{r}^{\prime}=\bar{r}+R \bar{\Omega} \ldots \ldots 10$

2. Particle Incoming Direction and Two Possible Scattering Directions at a Point $\overline{\mathrm{P}}(\mathrm{X}, \mathrm{Y}, \mathrm{Z}) \ldots \ldots$

3. The $P_{3}$ Scattering Distribution and the Discrete

Distribution for Group 1 to Group 1 Transfer . . . . . 20

4. The $P_{3}$ Scattering Distribution and the Discrete Distribution for Group 4 to Group 4 Transfer .... . . . . . . . 21

5. A Typical Particle Flight Trajectory in the Cylindrical Geometry which is Divided into Twelve Spatial Regions . . 27

6. Geometry of Concrete Cylinder with Axial Duct, Source, Detectors, and Adjoint Sources ......... 31

7. The Components of the Direction Vector $\bar{\Omega}$ in the Rectangular and Cylindrical Coordinate Systems . . . . . . . . 36

8. Flow Diagram of Generation of Importance Functions from the Adjoint DOT Calculation and Application to MORSE

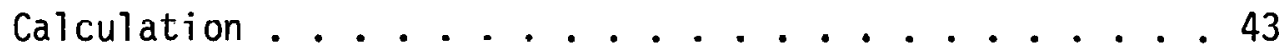

9. Source Location Importance Function from the Adjoint DOT

Calculation with Axial Adjoint Source, and the Approximating Function $I_{s}(R)$ to the Importance Function .................... 4 47

10. Curvature Effect of a Particle Direction in a Cylindrical Geometry ................ 52 
FIGURE

PAGE

11. Distribution of Neutron Collision Sites for the (0.5)

Calculation .............. 64

12. Distribution of Neutron Collision Sites for the (S)

Calculation ............... 65

13. Distribution of Neutron Collision Sites for the (P)

Calculation ................. 66

14. Distribution of Neutron Collision Sites for the (A)

Calculation ............. 67

15. Distribution of Neutron Collision sites for the (SP)

Calculation................. 68

16. Distribution of Neutron Collision Sites for the (SA)

Calculation ................. 69

17. The Direction Vector $\bar{\Omega}$ and Its Components . . . . . 102

18. Particle Trajectories in the Geometry of the Standard Problem .............. 107

19. Total Adjoint Flux Contour of the DOT Calculation with Axial Adjoint Source .......... . 110

20. Total Adjoint Flux Versus Z-Axis in the Duct of the DOT Calculation with Axial Adjoint Source . . . . . . 111

21. Total Adjoint Flux Versus Radius at the Bottom Surface of the Cylinder of the DOT Calculation with Axial Adjoint Source . . . . . . . . . . . 112

22. Total Mdjoint Flux Levels of the DOT Calculation with Axial Adjoint Source ............ 113 
FIGURE

PAGE

23. Group 1 Adjoint Flux Levels of the DOT Calculation with

Axial Adjoint Source ............. . 114

24. Group 5 Adjoint Flux Levels of the DOT Calculation with

Axial Adjoint Source ............. 115

25. Group 10 Adjoint Flux Levels of the DOT Calculation with

Axial Adjoint Source ............. 116

26. Group 14 Adjoint Flux Levels of the DOT Calculation with

Axial Adjoint Source . . . . . . . . . 117

27. Total Adjoint Flux Contour of the DOT Calculation with

Off-Axial Adjoint Source . . . . . . . . . 118

28. Total Adjoint Flux Versus Z-Axis in the Duct of the DOT

Calculation with Off-Axial Adjoint Source . . . . . 119

29. Total Adjoint Flux Versus Radius at the Bottom Surface

of the Cylinder of the DOT Calculation with Off-Axial

Adjoint Source .............. 120

30. Total Adjoint Flux Levels of the DOT Calculation with

Off-Axial Adjoint Source ........... 121

31. Group 1 Adjoint Flux Levels of the DOT Calculation with

Off-Axial Adjoint Source . . . . . . . . . 122

32. Group 5 Adjoint Flux Levels of the DOT Calculation with

Off-Axial Adjoint Source . . . . . . . . . . 123

33. Group 10 Adjoint Flux Levels of the DOT Calculation with

off-Axial Adjoint Source . . . . . . . . 124

34. Group 14 Adjoint Flux Levels of the DOT Calculation with

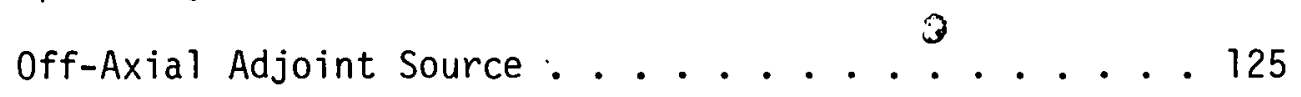




\section{CHAPTER I}

\section{INTRODUCTION}

The Monte Carlo method is a very useful tool to solve a large class of radiation transport problems. In analyzing radiation transport by Monte Carlo, enough histories must be generated and processed in order to sufficiently determine the average behavior of the particles. For the deep penetration problem where the natural probability of contribution to the answer of interest is small, importance sampling must be used. Importance sampling is a variance reduction procedure which alters the sampling scheme to one which samples more often from the phase space coordinates which make important contributions to the answer. In Monte Carlo, importance sampling is a form of "biasing."

It has long been recognized $(1,2,3)$ that the adjoint solution is a good if not optimum choice of importance function for Monte Carlo biasing. In principle, the application of importance functions derived from adjoint calculations is straightforward. However, in pract1ce, it has been limited tn special cases where either a reasonable approximation to the importance function may be represented analytically or the adjoint flux can be numerically calculated from a simplified problem.

Kalos ${ }^{(4)}$ used an approximate analytic form of importance function to bias neutron penetration through hydrogen slabs. Armstrong and Stevens ${ }^{(5)}$ applied the $v^{0}$ importance function, which is the first 
term of a series representation of the adjoint function and is similar to the form used by Kalos, to calculate gamma ray penetration in slabs of lead and of water.

It should be noted that the. works of Kalos, and Armstrong and Stevens concerned only special cases where the importance function could be approximated by a relatively simple analytic expression. Another approach to obtaining an importance function is to solve the inhomogeneous adjoint transport equation and to use this adjoint flux as the importance function. Since it is just as difficult to calculate the numerical adjoint flux as it is to calculate the forward flux, a practical approach is to solve the adjoint problem in a simplified geometry. This approximate adjoint flux is then employed as the importance function in the Monte Carlo calculation of the real problem.

Benda11 and Mccracken ${ }^{(6)}$ calculated the numerical adjoint flux with the removal diffusion method, then used this adjoint flux in the Monte Carlo calculation with some success. Cain ${ }^{(7)}$ used the onedimensional discrete ordinates adjoint flux to bias the source energy distribution and to generate parameters for an exponential transform which is dependent on energy and position. Schmidt et al. (8) extended this approach to include directional dependence in the exponential transform parameters, and at the same time utilized the adjoint function as a guide to set the weight standards of Russian roulette and splitting.

Burgart and Stevens ${ }^{(9)}$ further extended the application of the one-dimensional discrete ordinates adjoint flux to bias the 
collision kernel. A symmetrical discrete angular grid was employed to incorporate angular biasing and angular-dependent path length stretching. This method was quite successful for deep penetration in simple geometries. However, the application of this approach to deep penetration in complex geometries such as a thick concrete shield with a penetrating duct is very limited. Two reasons account for this limitation:

1. The one-dimensional discrete ordinates adjoint flux does not lead to a good approximation of the importance function for the three-dimensional complex geometry problem.

2. The symmetrical discrete angular grid, which restricts particles to travel only in discrete directions, fails to properly describe the streaming radiation through the duct.

The objective of the present study is to use the two-dimensional discrete ordinates adjoint flux to bias the Monte Carlo analys is of the three-dimensional deep penetration problem. Two biasing techniques, "angular prohahility hiasing" and "path' length biasing," have been developed. The effectiveness of "source biasing" using the scalar adjoint flux was also investigated.

In the angular probability biasing technique, the probabilities associated with the scattering directions are biased by the point value. The point value is used because it is the value to the effect of interest of a particle coming out of a collision. In the path length biasing technique, the selection of the next collision 
site is biased by the event value. The event value, being the value of a particle going into a collision, is the appropriate function to bias the path length of a particle going into the next collision site. These two value functions can be obtained directly from a two-dimensional discrete ordinates adjoint calculation.

The three biasing schemes are investigated for neutron transport in a thick concrete shield with an axial duct. Results of biased Monte Carlo calculations are compared with standard Monte Carlo and discrete ordinates calculations. Improvements achieved by the biasing schemes are very good, and their general usefulness is clearly demonstrated.

The multigroup Monte Carlo code, MORSE $(10)$ is used for the Monte Carlo calculations, and the two-dimensional discrete ordinates code, $\mathrm{DOT}^{(11)}$ is used to calculate the adjoint fluxes. 
CHAPTER II

THE ADJOINT BOLTZMANN TRANSPORT EQUATIONS

In this chapter, the time-independent multigroup adjoint integral transport equations are formulated in terms of the point value and the event value importance functions.* Then the adjoint Boltzmann integrodifferential transport equation is derived from the principle of conservation of value. Finally, the relationship between the point value and the event value and their applications to the biased Monte Carlo calculation are discussed.

\subsection{The Event Value and the Point Value Equations}

There are several integral forms to the adjoint Boltzmann transport equation, namely the point value equation, the event value equation, the emergent adjunction density equation, and the adjunction

event density equation. (10) These adjoint integral transport equations describe the importance of radiation particles with respect to a specific effect of interest. Hence, they are equivalent to one another even though each involves a different variable. The focus of this study is on the event value and the point value because they are the importance functions which will be used to bias the Monte Carlo random walk. In this section, the integral transport equations for the event value and the point value will be formulated.

* For brevity, these quantities will be referred to simply as the point value and the event value respectively. 
The behavior of radiation particles in a medium may be described in terms of the event density or the emergent particle density. The event density is the density of particles going into collisions, and the emergent particle density is the density of particles leaving a source or emerging from collisions. The concept of the importance of particles can be described in terms of the value of going into a collision or in terms of the value of emerging from a collision or a source. The event value $W_{g}(\bar{r}, \bar{\Omega})^{\dagger}$ is defined to be the value to the effect of interest of a particle which enters a collision at point $\bar{r}$ with energy group $g$ and direction $\bar{\Omega}$. The point value $x_{g}^{\star}(\bar{r}, \bar{\Omega})$ is defined to be the value of a particle which emerges from a collision or from a source at point $\bar{r}$ with energy group $g$ and direction $\bar{\Omega}$. According to Irving, ${ }^{(12)}$ the event value $w_{g}(\bar{r}, \bar{\Omega})$ is composed of two parts, the immediate payoff and the future payoff. The immediate payoff is simply the group $g$ response function $\mathrm{P}_{\mathrm{g}}^{\psi}(\bar{r}, \bar{\Omega})$. The future payoff is the expected value* of the probability that the particle survives the collision and emerges with energy group $g^{\prime}$ and direction in $\mathrm{d}_{\bar{\Omega}}^{-}$about $\bar{\Omega}^{-}$times the value of this emergent particle $x_{g^{*}}^{*}\left(\bar{r}, \bar{\Omega}^{-}\right)$. This interpretation leads to the following time-independent multigroup integral equation

$$
W_{g}(\bar{r}, \bar{\Omega})=P_{g}^{\psi}(\bar{r}, \bar{\Omega})+\sum_{g^{-}} \int_{4 \pi} \frac{\sum_{s}^{g \rightarrow g^{\prime}}\left(\bar{r}, \bar{\Omega}^{\prime} \rightarrow \bar{\Omega}^{-}\right)}{\sum_{t}^{g}(\bar{r})} x_{g^{-}}^{*}\left(\bar{r}, \bar{\Omega}^{-}\right) d \bar{\Omega}^{-},
$$

\footnotetext{
The symbolism and terminology of this chapter follow those of Straker et al. (10)

*Expected value implies integration over all appropriate phase space coordinates.
} 
where

$$
\begin{aligned}
\mathrm{P}_{g}^{\psi}(\bar{r}, \bar{\Omega})= & \text { the group } \mathrm{g} \text { response function for } \bar{\Omega} \text {-directed } \\
& \text { particles which experience events at } \bar{r}, \\
\sum_{t}^{g}(r)= & \text { the group } g \text { total macroscopic cross section }\left[\mathrm{cm}^{-1}\right], \\
\sum_{S}^{g \rightarrow g^{-}}\left(\bar{r}, \bar{\Omega}^{\prime} \rightarrow \bar{\Omega}^{-}\right)= & \text {the group } g \text { to group } g^{-} \text {differential } \\
& \text { scattering cross section }\left[\mathrm{cm}^{-1} \text { steradian }{ }^{-1}\right] .
\end{aligned}
$$

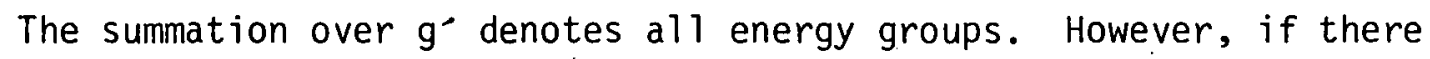
is no upscatter, the $g^{-}$summation would be from $g$ to $G$, where $G$ is the group number with lowest energy.

As defined above, the point value $x_{g}^{*}(\bar{r}, \bar{\Omega})$ is the value of a particle which emerges from a collision or from a source at $\bar{r}$ with energy group $\mathrm{g}$ and direction $\bar{\Omega}$. This particle will experience an event in $d R$ about $\bar{r}+R \bar{\Omega}$ with the probability

$$
\Sigma_{t}^{g}(\bar{r}+R \bar{\Omega}) e^{-\int_{0}^{R} \Sigma_{t}^{g}\left(\bar{r}+R^{-} \bar{\Omega}\right) d R^{\prime}} d R
$$

and the value of this event is given by $w_{g}(\bar{r}+R \bar{\Omega}, \bar{\Omega})$. Since the value of leaving a collision is equal to the expected value of going into future collisions, the following time-independent multigroup integral equation is obtained.

$$
x^{*}(\bar{r}, \bar{\Omega})=\int_{0}^{\infty} \Sigma_{t}^{g}(\bar{r}+R \bar{\zeta}) e^{-\int_{0}^{R} \sum_{t}^{g}\left(\bar{r}+R^{-} \bar{\Omega}\right) d R^{\prime}} W_{g}(\bar{r}+R \bar{\Omega}, \bar{\Omega}) d R .
$$

Equations (2-1) and (2-2) express explicitly the event value and the point value respectively in terms of the other. The integral transport equation for the event value is obtained by substituting Equation (2-2) into Equation $(2-1)$. 


$$
\begin{aligned}
& W_{g}(\bar{r}, \bar{\Omega})=P_{g}^{\psi}(\bar{r}, \bar{\Omega})+\sum_{g}, \int_{4 \pi} \int_{0}^{\infty} \frac{\Sigma_{s}^{g \rightarrow g^{-}}\left(\bar{r}, \bar{\Omega}^{\prime} \rightarrow \bar{\Omega}^{-}\right)}{\Sigma_{t}^{g}(\bar{r})} x \\
& \Sigma_{t}^{g^{\prime}}\left(\bar{r}+R^{\prime} \bar{\Omega}^{\prime}\right) e^{-\int_{0}^{R} \Sigma_{t}^{g^{\prime}}\left(\bar{r}+R^{-} \bar{\Omega}^{\prime}\right) d R^{\prime}} W_{g^{-}}\left(\bar{r}+R^{-} \bar{\Omega}^{-}, \bar{\Omega}^{-}\right) \mathrm{dRd} \bar{\Omega}^{\prime} .
\end{aligned}
$$

Substitution of Equation (2-1) into Equation (2-2) yields the integral transport equation for the point value

$$
\begin{aligned}
& x_{\mathrm{g}}^{\star}(\bar{r}, \bar{\Omega})=\int_{0}^{\infty} \Sigma_{\mathrm{t}}^{g}(\bar{r}+R \bar{\Omega}) e^{-\int_{0}^{R} \Sigma_{\mathrm{t}}^{g}\left(\bar{r}+R^{-} \bar{\Omega}\right) d R^{\prime}}\left[\mathrm{P}_{\mathrm{g}}^{\psi}(\bar{r}+R \bar{\Omega}, \bar{\Omega})+\right. \\
& \left.\sum_{g^{-}} \int_{4 \pi} \frac{\sum_{\mathrm{s}}^{g \rightarrow g^{-}}\left(\bar{r}+R \bar{\Omega}, \bar{\Omega}^{\prime} \rightarrow \bar{\Omega}^{-}\right)}{\sum_{t}^{g}(\bar{r}+R \bar{\Omega})} x_{g^{-}}^{*}\left(\bar{r}+R \bar{\Omega}, \bar{\Omega}^{-}\right) d \bar{\Omega}^{-}\right] d R \\
& =\underset{g}{\mathrm{P}}(\bar{r}, \bar{\Omega})+\sum_{g^{\prime}} \int_{4 \pi} \int_{0}^{\infty} \Sigma_{t}^{g}(\bar{r}+R \bar{\Omega}) e^{-\int_{0}^{R} \Sigma_{t}^{g}\left(\bar{r}+R^{-} \bar{\Omega}\right) d R^{-}} x \\
& \frac{\sum_{s}^{g \rightarrow g^{\prime}}\left(\bar{r}+R \bar{\Omega}, \bar{\Omega}_{\Omega} \rightarrow \bar{\Omega}^{\prime}\right)}{\Sigma_{t}^{g}\left(\bar{r}+R_{\bar{\Omega}}\right)} x_{g^{-}}^{\star}\left(\bar{r}+R_{\bar{\Omega}}, \bar{\Omega}^{-}\right) \mathrm{d} \bar{\Omega}-\mathrm{d} R,
\end{aligned}
$$

where $\mathrm{P}_{g}^{\mathrm{X}}(\bar{r}, \bar{\Omega})=\int_{0}^{\infty} \Sigma_{t}^{g}(\bar{r}+R \bar{\Omega}) e^{-\int_{0}^{R} \sum_{t}^{g}\left(\bar{r}+R^{-} \bar{\Omega}\right) d R^{-}} P_{g}^{\psi}(\bar{r}+R \bar{\Omega}, \bar{\Omega}) d R$.

$\mathrm{P}_{\mathrm{g}}^{\mathrm{X}}(\bar{r}, \bar{\Omega})$ is the response function of a particle which emerges from a collision at $\bar{r}$ with energy group $g$ and direction $\bar{\Omega}$. Then according to Equation (2-5), the response function of a particle leaving a collision at $\bar{r}$ is the expected value of the probability of traveling 
to $\bar{r}^{-}=\bar{r}+R \bar{\Omega}$ and having an event within $d R$ about $\bar{r}^{-}$times the response function for an event at $\bar{r}^{-}=\bar{r}+R \bar{\Omega}$. Figure 1 illustrates the relationship between spatial points $\vec{r}$ and $\bar{r}^{\prime}$.

\subsection{Derivation of the Adjoint Boltzmann Equation from the Value}

\section{Functions}

The multigroup adjoint Boltzmann transport equation is well documented in the literature, $(10,12,13)$ and can be written as

$$
\begin{aligned}
& -\bar{\Omega} \cdot \nabla \phi_{g}^{*}(\bar{r}, \bar{\Omega})+\sum_{t}^{g}(\bar{r}) \phi_{g}^{*}(\bar{r}, \bar{\Omega})= \\
& S_{g}^{\star}(\bar{r}, \bar{\Omega})+\sum_{g^{-}} \int_{4 \pi} \sum_{\mathrm{s}}^{g \rightarrow g^{-}}\left(\bar{r}, \bar{\Omega}^{\prime} \rightarrow \bar{\Omega}^{-}\right) \phi_{g^{-}}^{*}\left(\bar{r}, \bar{\Omega}^{-}\right) \mathrm{d} \bar{\Omega}^{-},
\end{aligned}
$$

where

$$
\begin{aligned}
S_{g}^{\star}(\bar{r}, \bar{\Omega})= & \text { the group } g \text { adjoint source, } \\
\phi_{g}^{\star}(\bar{r}, \bar{\Omega})= & \text { the group g adjoint angular flux the nature of which } \\
& \text { is determined by the choice of the adjoint source } \\
& S_{g}^{\star}(\bar{r}, \bar{s}) .
\end{aligned}
$$

Equation (2-6) can be derived from the forward Boltzmann transport equation by defining an adjoint function and an operator which is adjoint to the operator associated with the forward Boltzmann transport equation. Then from the property of adjoint operators and the proper application of the boundary conditions, the adjoint Boltzmann transport equation can be obtained. This is strictly a mathematical procedure, and the physical meaning of the adjoint function is yet to be determined. The details of this derivation are presented in References 10 and 13. 


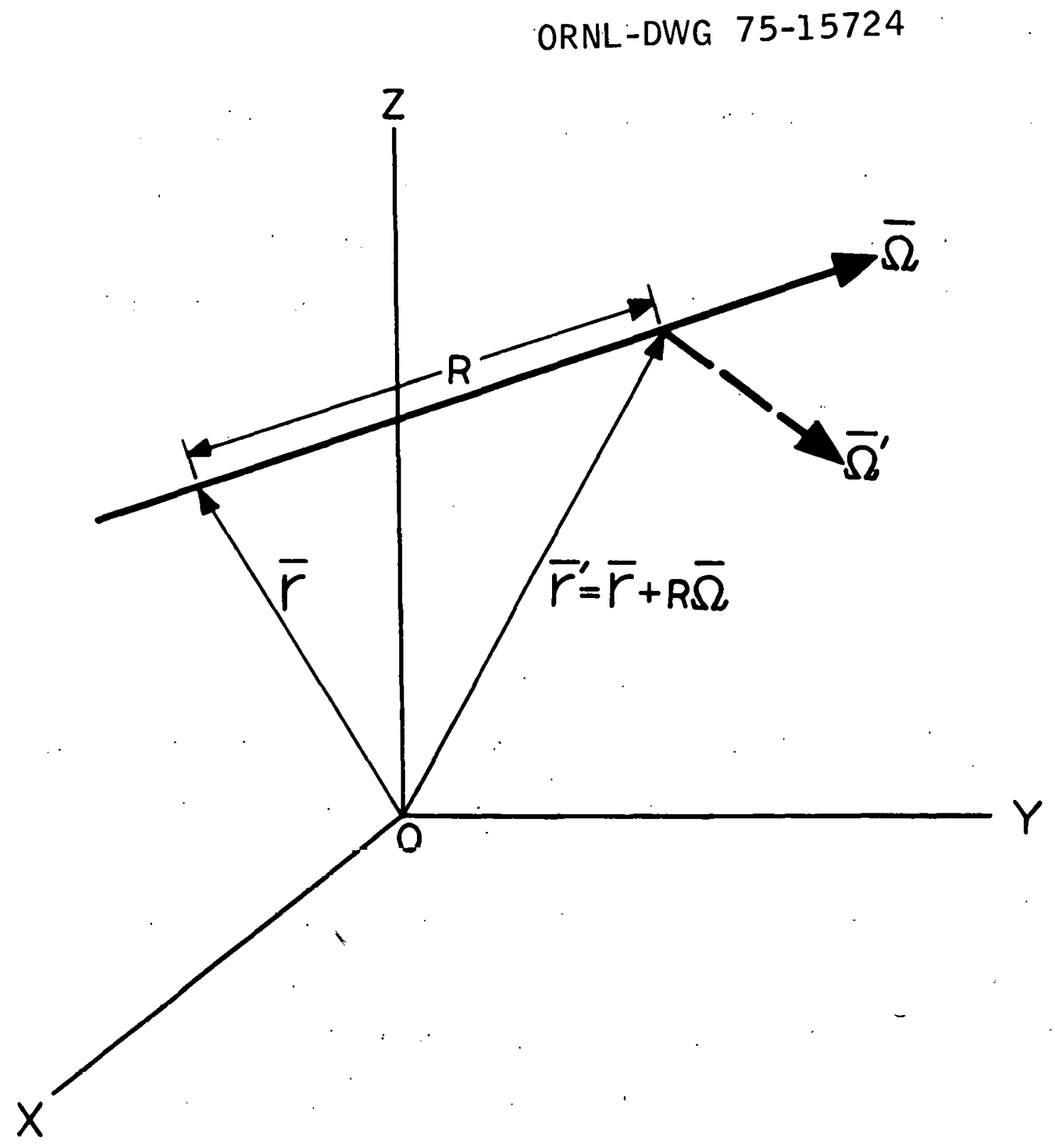

Figure 1. Coordinate System which Relates a Fixed Point $\bar{r}$ to an Arbitrary Point $\bar{r}^{\prime}=\bar{r}+R \bar{\Omega}$. 
Using the physical interpretation of the adjoint function as a neutron importance to an effect of interest, Bell and Glasstone (13 derived directly the adjoint Boltzmann transport equation from first principles.

The adjoint Boltzmann transport equation can also be derived by requiring that the value of a particle be conserved along its path. The derivation is based on the distinction between the value of a particle entering a collision and the value of that particle leaving a collision. Consider a particle which emerges from a point $\bar{r}$ in direction $\bar{s}$ and energy group $g$. For the particle's value to be conserved, the following relationship must be true for an incremental distance $\Delta R$ along the $\bar{\Omega}$ direction:

$$
\begin{gathered}
{\left[\begin{array}{l}
\text { value of emerging } \\
\text { from point } \bar{r}
\end{array}\right]=\left[\begin{array}{c}
\text { probability of } \\
\text { collision in } \Delta R
\end{array}\right]\left[\begin{array}{l}
\text { value of going into } \\
\text { collision in } \Delta R
\end{array}\right]} \\
+\left[\begin{array}{c}
\text { probability of no } \\
\text { collision in } \Delta R
\end{array}\right]\left[\begin{array}{c}
\text { value of emerging } \\
\text { from point } \bar{r}+\Delta R \bar{\Omega}
\end{array}\right],
\end{gathered}
$$

which can be expressed as

$$
\begin{gathered}
x_{g}^{*}(\bar{r}, \bar{\Omega})=\left[\Sigma_{t}^{g}(\bar{r}) \Delta R\right]\left[W_{g}\left(\bar{r}+\frac{1}{2} \Delta R \bar{\Omega}, \bar{\Omega}\right)\right]+\left[1-\Sigma_{t}^{g}(\bar{r}) \Delta R\right] \\
{\left[x_{g}^{\star}(\bar{r}+\Delta R \bar{\Omega}, \bar{\Omega})\right] .}
\end{gathered}
$$

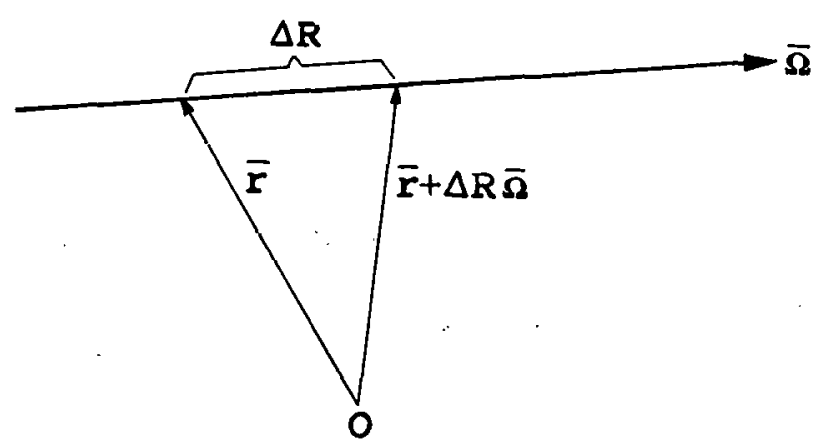


Equation $(2-7)$ can be rearranged as

$$
\begin{array}{r}
\frac{x_{g}^{\star}(\bar{r}, \bar{\Omega})-x_{g}^{\star}(\bar{r}+\Delta R \bar{\Omega}, \bar{\Omega})}{\Delta R}=\Sigma_{t}^{g}(\bar{r}) w_{g}\left(\bar{r}+\frac{1}{2} \Delta R \bar{\Omega}, \bar{\Omega}\right)- \\
\sum_{t}^{g}(\bar{r}) x_{g}^{\star}(\bar{r}+\Delta R \bar{\Omega}, \bar{\Omega}) .
\end{array}
$$

Taking the limit of $\Delta R \rightarrow 0$, gives

$$
-\frac{d x_{g}^{*}(\bar{r}, \bar{\Omega})}{d R}=\Sigma_{t}^{g}(\bar{r}) w_{g}(\bar{r}, \bar{\Omega})-\Sigma_{t}^{g}(\bar{r}) x_{g}^{*}(\bar{r}, \bar{\Omega})
$$

and with rearrangement Equation (2-8) becomes

$$
x_{g}^{*}(\bar{r}, \bar{\Omega})-\frac{1}{\sum_{t}^{g}(\bar{r})} \frac{d x_{g}^{*}(\bar{r}, \bar{\Omega})}{d R}=w_{g}(\bar{r}, \bar{\Omega}) \text {. }
$$

Noting that $\frac{d x_{g}^{*}(\bar{r}, \bar{\Omega})}{d R}=\bar{\Omega} \cdot \nabla x_{g}^{*}(\bar{r}, \bar{\Omega})(10)$ and substituting $w_{g}(\bar{r}, \bar{\Omega})$ from Equation (2-1) into Equation (2-3) yields

$$
\begin{gathered}
-\bar{\Omega} \cdot \nabla x_{g}^{\star}(\bar{r}, \bar{\Omega})+\sum_{t}^{g}(\bar{r}) x_{g}^{\star}(\bar{r}, \bar{\Omega}) \\
=P_{g}^{\phi}(\bar{r}, \bar{\Omega})+\sum_{g^{-}} \int_{4 \pi^{2}} \sum_{s}^{g \rightarrow g^{-}}\left(\bar{r},{\bar{\Omega} \rightarrow \bar{\Omega}^{-}}^{-}\right) x_{g^{-}}^{\star}\left(\bar{r}, \bar{\Omega}^{-}\right) \mathrm{d} \bar{\Omega}^{-},
\end{gathered}
$$

where $\mathrm{P}_{g}^{\phi}(\bar{r}, \bar{\Omega})=\Sigma_{t}^{g}(\bar{r}) P_{g}^{\psi}(\bar{r}, \bar{\Omega})$.

$\mathrm{P}_{\mathrm{g}}^{\phi}(\bar{r}, \bar{\Omega})$ is the group $\mathrm{g}$ response function of the effect of interest due to a unit angular flux. A comparison of Equation (2-10) with the multigroup adjoint transport equation as given by Equation $(2-6)$ reveals that if $\mathrm{P}_{\mathrm{g}}^{\phi}(\bar{r}, \bar{\Omega})=\mathrm{S}_{\mathrm{g}}^{*}(\bar{r}, \bar{\Omega})$, the two equations are identical 
and $x_{g}^{\star}(\bar{r}, \bar{\Omega}) \equiv \phi_{g}^{\star}(\bar{r}, \bar{\Omega})$. Therefore, the adjoint angular flux (the solution of the integro-differential form of the adjoint Boltzmann equation) is the value of a particle leaving a collision if the adjoint source is taken to be the response function $\mathrm{P}_{g}^{\phi}(\bar{r}, \bar{\Omega})$.

\subsection{Application of the Point Value and the Event Value in Monte} Carlo Biasings

The random walk procedure in Monte Carlo calculations is composed of the transport process and the collision process. The biasing of these processes requires importance functions which may be obtained from appropriate deterministic adjoint calculations.

Since the transport process involves the selection of the next collision site, the event value would seem to be the appropriate importance function to bias the transport kernel. In the collision process, a particle emerges from a collision site with its new energy and direction determined by the collision kernel. The point value would seem to be the suitable importance function to bias the collision kernel. Therefore, a distinction must be made between the point value $x^{*}$ and the event value $W$, insofar as their applications to Monte Carlo biasing techiniques are concerned. Finally, proper correction of the particles' statistical weights must be exercised in order to remove the bias and preserve the "fair game." 


\section{CHAPTER III}

\section{DEVELOPMENT OF MONTE CARLO IMPORTANCE SAMPLING TECHNIQUES}

This chapter first presents a description of the collision mechanics used in the Monte Carlo code MORSE. (10) The "angular probability biasing" technique is then introduced, and the application of the point value to bias the selection of the emergent direction from a collision process is described. Finally, "the path length biasing" technique using the event value as the importance function is developed and a method of normalizing the altered transport kernel is formulated.

\subsection{Collision Mechanics}

The MORSE code utilizes the same multigroup cross sections used by the discrete ordinates codes DOT, (11) ANISN, (19) or DTF-IV. (20) These cross section sets employ an $n^{\text {th }}$-order Legendre polynomial expansion to describe the scattering distribution for each group-togroup transfer.

At a collision site, the emergent energy group and direction are normally selected according to the natural collision kernel

$$
c^{g \rightarrow g^{-}}\left(\bar{r}, \bar{\Omega} \rightarrow \bar{\Omega}^{-}\right)=\frac{\sum_{s}^{g \rightarrow g^{\prime}}\left(\bar{r}, \bar{\Omega}^{\prime} \rightarrow \bar{\Omega}^{-}\right)}{\sum_{s}^{g}(\bar{r})}
$$

where $\Sigma_{\mathrm{S}}^{g \rightarrow g^{-}}\left(\vec{r}, \vec{\Omega}_{\rightarrow} \rightarrow \bar{\Omega}^{-}\right)$is the group $g$ to group $g^{-}$differential scattering cross section and the group scattering cross section $\Sigma_{s}^{g}(\bar{r})$ is equal to 
$\sum_{g^{-}} \int_{4 \pi} \sum_{S}^{g_{S}^{\rightarrow g^{-}}\left(\bar{r}, \bar{\Omega}^{\prime} \rightarrow \bar{\Omega}^{-}\right)} d \bar{\Omega}^{-}$. The collision kernel is a properly normalized joint probability density function (p.d.f.) and may be expressed as a product of a marginal p.d.f. and a conditional p.d.f.

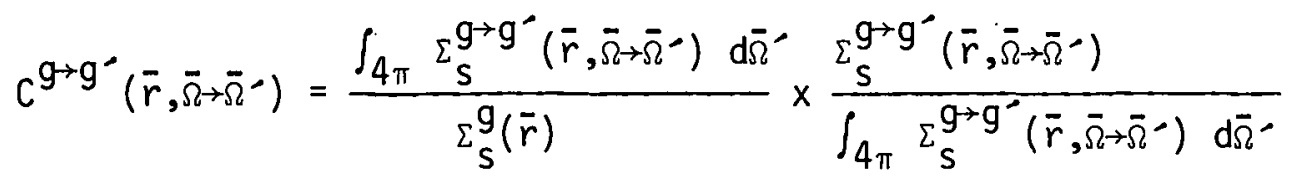

$$
\begin{aligned}
& =\frac{\Sigma_{S}^{g \rightarrow g^{\prime}}(\bar{r})}{\Sigma_{S}^{g}(\bar{r})} \frac{\sum_{S}^{g \rightarrow g^{\prime}}\left(\bar{r}, \bar{\Omega} \rightarrow \bar{\Omega}^{\prime}\right)}{\sum_{S}^{g^{\rightarrow} g^{\prime}}(\bar{r})} .
\end{aligned}
$$

The energy group $g^{-}$of the emergent particles is selected from the marginal p.d.f.

$$
\frac{\sum_{S}^{g \rightarrow g^{\prime}}(\bar{r})}{\Sigma_{S}^{g}(\bar{r})}, \text { for } g^{-}=g, g+1, \ldots . G
$$

The emergent direction $\bar{\Omega}^{-}$is then selected from the conditional p.d.f.

$$
\frac{\sum_{s}^{g \rightarrow g^{-}}\left(\bar{r}, \bar{\Omega}^{\prime} \rightarrow \bar{\Omega}^{-}\right)}{\sum_{s}^{g \rightarrow g^{\prime}}(\bar{r})}
$$

given that a particular $g \rightarrow g^{-}$transfer has been selected according to Equation (3-3). Considering the scattering process as azimuthal symmetric, the differential scattering cross section may be written as a function of the cosine of the polar anglc, and expanded as a serics of Legendre polynomtals

$$
\sum_{s}^{g \rightarrow g^{\prime}}\left(\bar{r}, \bar{\Omega}^{\prime} \rightarrow \bar{\Omega}^{-}\right)=\frac{1}{4 \pi} \sum_{\ell=0}^{n} f_{\ell}^{g \rightarrow g^{-}}(\bar{r}) P_{\ell}(\mu),
$$


where

$$
\begin{aligned}
f_{\ell}^{g \rightarrow g^{-}}(\bar{r})= & \text { the } \ell^{\text {th }} \text { Legendre coefficient for the group } g \text { to group } \\
& g^{-} \text {transfer, } \\
n= & \text { the maximum order of the expansion, } \\
\mu= & \bar{\Omega} \cdot \bar{\Omega}^{-}, \text {the cosine of the scattering angle. }
\end{aligned}
$$

Using Equation (3-5), Equation (3-4) can be written as

$$
\frac{\sum_{S}^{g \rightarrow g^{\prime}}\left(\bar{r}, \bar{\Omega} \rightarrow \bar{\Omega}^{-}\right)}{\sum_{S}^{g^{\rightarrow \rightarrow g^{\prime}}}(\bar{r})}=\frac{1}{4 \pi \sum_{S}^{g^{g \rightarrow g^{\prime}}}(\bar{r})} \sum_{\ell=0}^{n} f_{\ell}^{g \rightarrow g^{-}}(\bar{r}) P_{\ell}(\mu)
$$

$$
=\frac{1}{2 \pi} \sum_{\ell=0}^{n} f_{\ell}^{f^{g \rightarrow g^{\circ}}}(\bar{r}) P_{\ell}(\mu)
$$

where $f_{l}^{\star^{g \rightarrow g^{\prime}}}(\bar{r})=\frac{f_{l}^{g \rightarrow g^{-}}(\bar{r})}{2 \Sigma_{s}^{g \rightarrow g^{\prime}}(\bar{r})}$.

The conditional p.d.f. as given by Equation (3-6) can be expressed as a product of two distributions, namely the polar angle distribution and the azimuthal angle distribution. The polar angle distribution is

$$
\omega(\mu)=\sum_{\ell=0}^{n} f_{\ell}^{\star g \rightarrow g^{\prime}}(\bar{r}) P_{\ell}(\mu),
$$

where the group-to-group transfer superscript and the position vector $\bar{r}$ are dropped to simplify the notation. Because of the azimuthally symmetric scattering, the azimuthal angle distribution is simply

$$
h(\phi)=\frac{1}{2 \pi} \text {. }
$$


The realization of the polar angle, $\cos ^{-1} \mu$, from Equation (3-7) is not accomplished directly. Instead, the distribution $\omega(\mu)$ is discretized by the use of a generalized Gaussian quadrature scheme. This approach effectively replaces the continuous distribution $\omega(\mu)$ by a discrete distribution $\omega^{\star}(\mu)$ with the constraint that the first $n$ moments of $\omega^{\star}(\mu)$ are identical to the first $n$ moments of $\omega(\mu)$ respectively. Thus, the angular distribution of Equation (3-6) becomes

$$
\begin{aligned}
\frac{\sum_{S}^{g \rightarrow g^{\prime}}\left(\bar{r}, \bar{\Omega}_{\Omega} \rightarrow \bar{\Omega}^{\circ}\right)}{\sum_{S}^{g \rightarrow g^{\prime}}(\bar{r})} & =h(\phi) \omega(\mu) \\
& =h(\phi) \omega^{\star}(\mu) \\
& =\frac{1}{2 \pi} \sum_{i=1}^{N} p_{i} \delta\left(\mu-\mu_{i}\right),
\end{aligned}
$$

where $p_{i}$ is the probability that polar angle $\cos ^{-1} \mu_{j}$ will be selected and $N$ is equal $(n+1) / 2$. The original normalization of Equation (3-6) gives

$$
\int_{4 \pi} \frac{\sum_{S}^{g \rightarrow g^{-}}\left(\bar{r}, \bar{\Omega}_{\rightarrow} \rightarrow \bar{\Omega}^{-}\right)}{\sum_{S}^{g^{\rightarrow} g^{\prime}}(\bar{r})} \mathrm{d} \bar{\Omega}^{-}=1,
$$

which provides the following nurmalization requirement. on the $p_{i}$ 's,

$$
\int_{0}^{2 \pi} \int_{-1}^{1} \frac{1}{2 \pi} \sum_{i=1}^{N} p_{i} \delta\left(\mu-\mu_{i}\right) d \mu d \phi=1
$$

and

$$
\sum_{i=1}^{N} p_{i}=1
$$


The selection of the outgoing direction is normaliy accomplished within MORSE in two separate steps:

1. First, a scattering polar angle is selected from the discrete distribution $\omega^{\star}(\mu)$.

2. Then an azimuthal angle is selected from the uniform distribution $h(\phi)$.

The sequence of the two steps can be reversed, i.e., first azimuthal angle then polar angle, because the two distributions, $\omega^{\star}(\mu)$ and $h(\phi)$, are independent. Figure 2 illustrates the two possible scattering directions $\bar{\Omega}_{1}$ and $\bar{\Omega}_{2}{ }_{2}$ and their corresponding cones with respect to the incoming flight direction $\bar{\Omega}$ for a $P_{3}$ scattering distribution.

The nature of the Gaussian quadrature scheme of discretizing the scattering distribution produces discrete directions which tend to be located near the peaks of the scattering distribution. Examples of the $P_{3}$ scattering distributions and their corresponding discrete distributions for the concrete cross section are shown in Figures 3 and 4. Note that although the truncated Legendre expansion for the scattering distribution may have negative values, the discrete distribution will always yield positive probabilities. This is true because the condition of positive probabilities is one of the constraints for the generalized Gaussian quadrature scheme. 


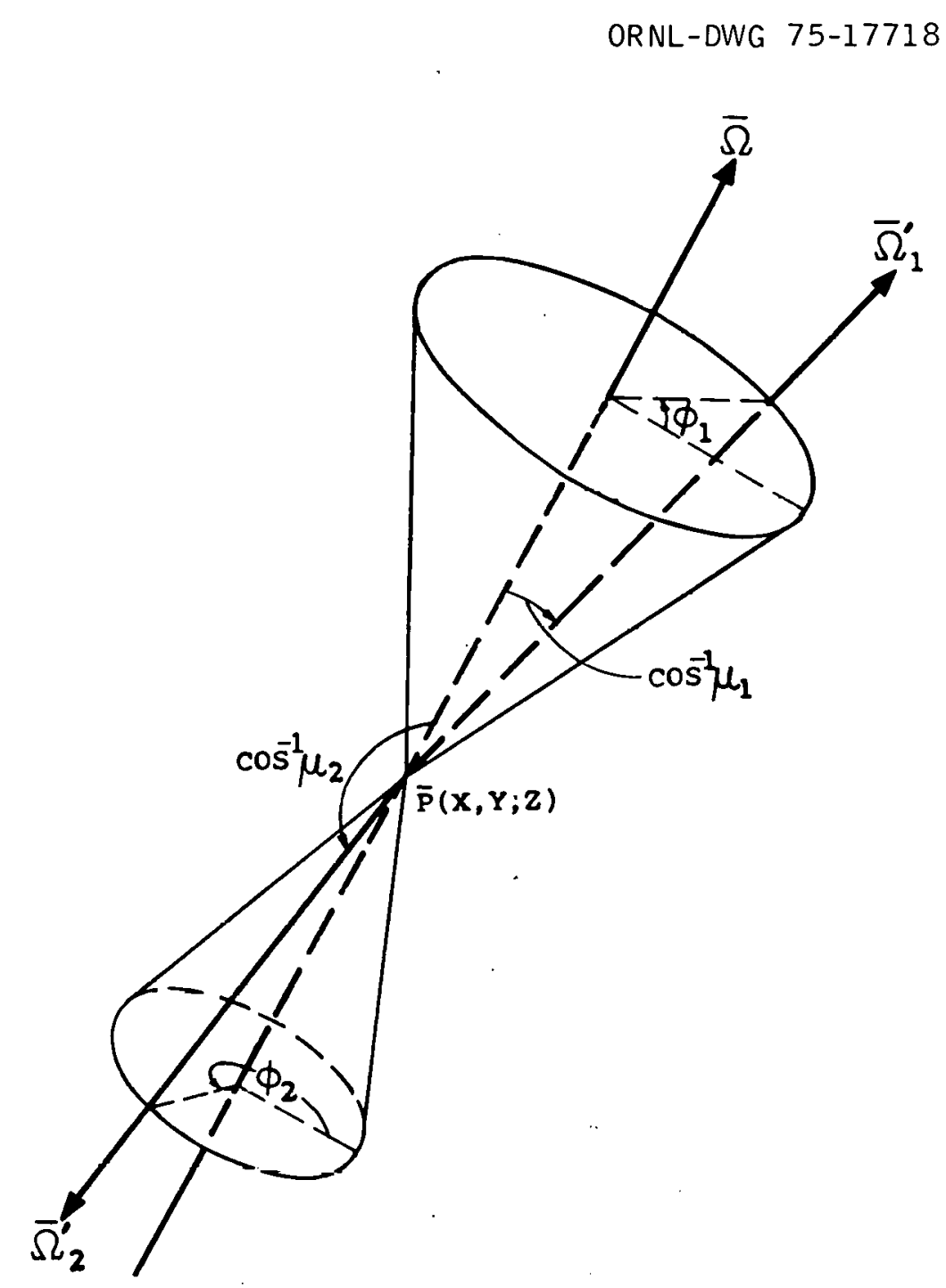

Figure 2. Particle Incoming Direction and Two Possible Scattering Directions at a Point $\mathrm{P}(\mathrm{X}, \mathrm{Y}, \mathrm{Z})$. 
ORNL-DWG. 75-15729

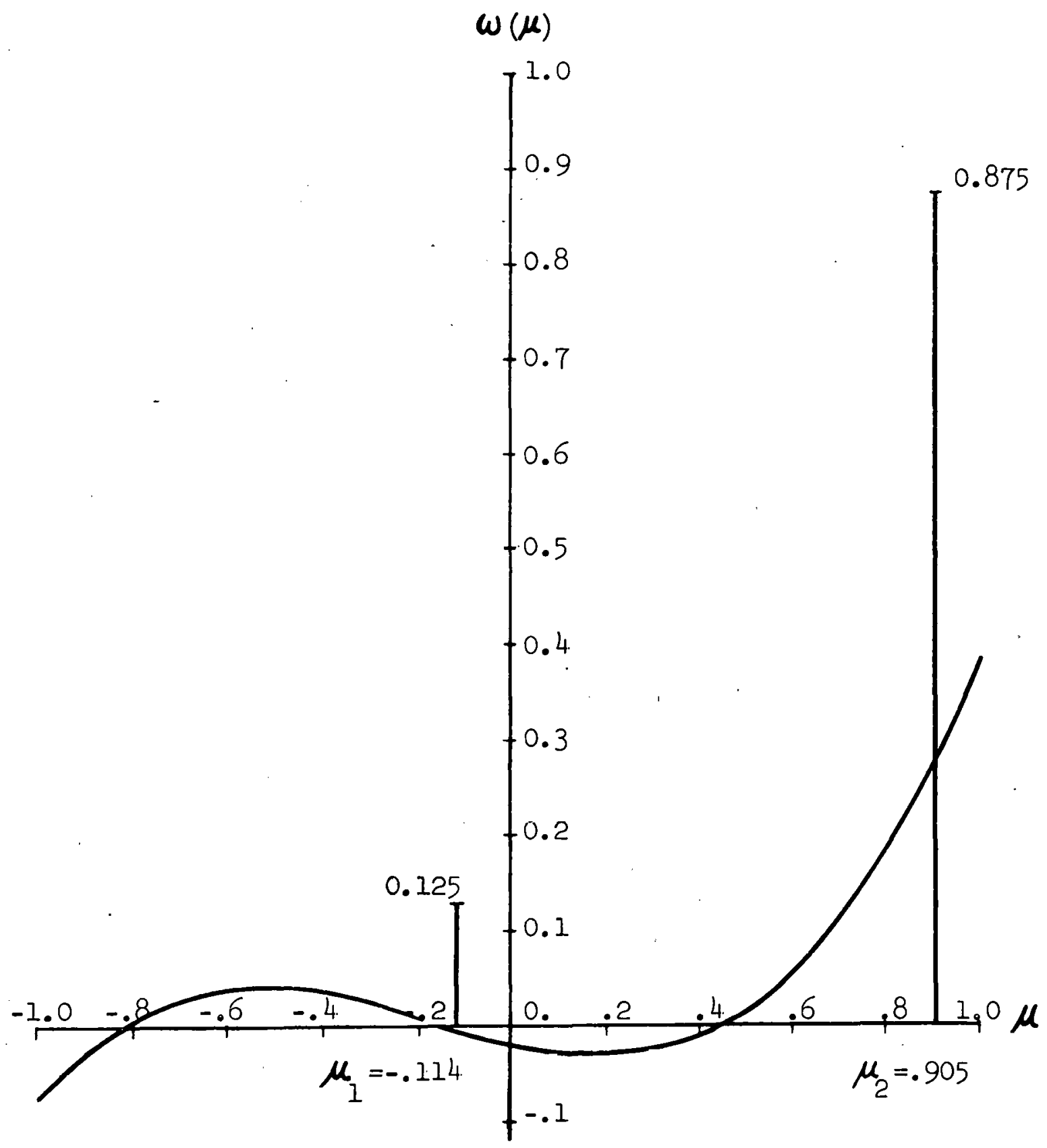

Figure 3. The $P_{3}$ Scattering Distribution and the Discrete Distribution for Group 1 to Group 1 .Transfer. 


\section{ORNL-DWG 75-15728}

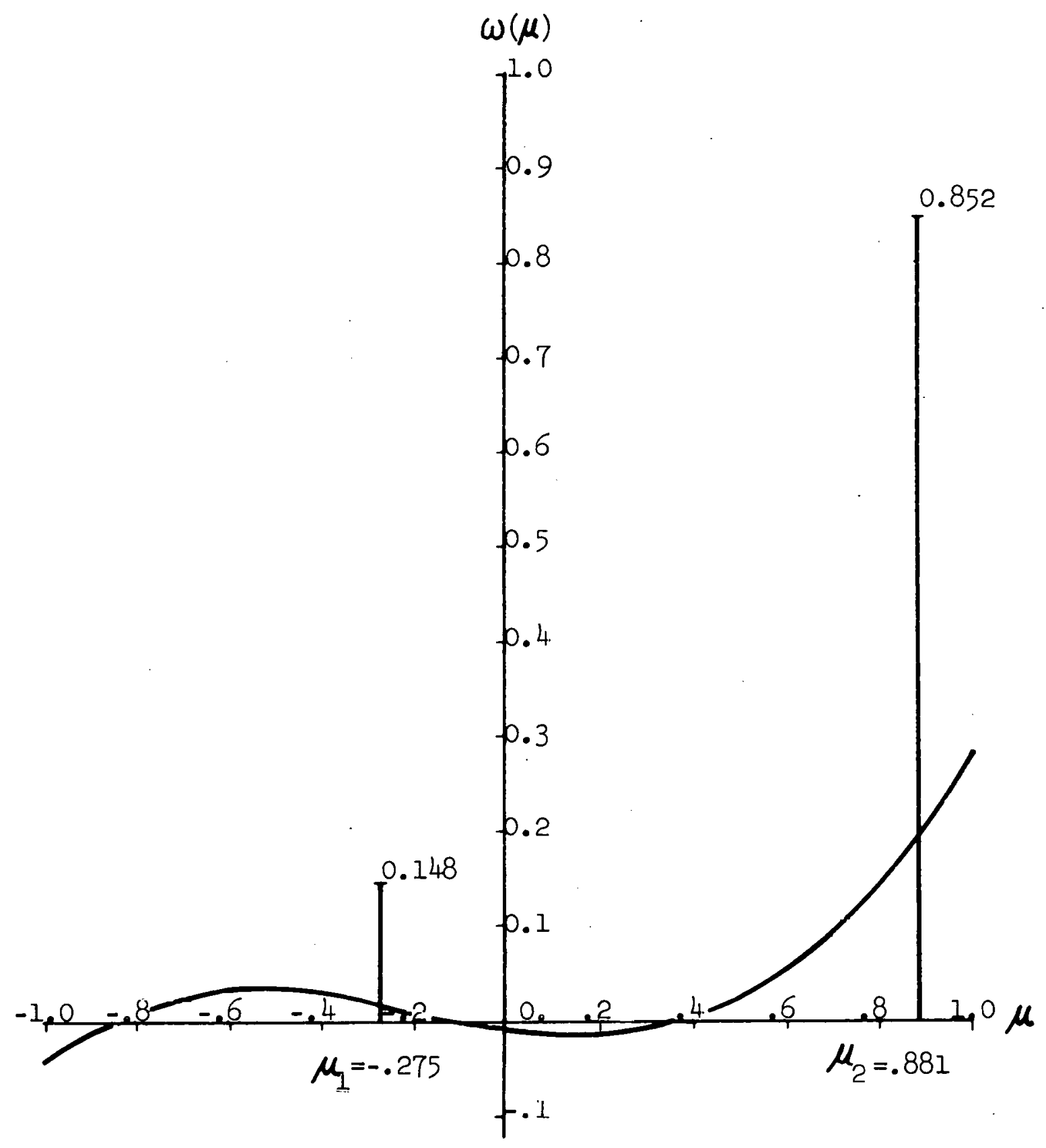

Figure 4. The $P_{3}$ Scattering Distribution and the Discrete Distribution for Group 4 to Group 4 Transfer. 


\subsection{The Angular Probability Biasing Technique}

The "angular probability biasing" technique is formulated by biasing the conditional p.d.f. of Equation (3-9) using the point value $x_{g}^{\star}(\bar{r}, \bar{\Omega})$ as the importance function. The biased scattering distribution is denoted as $\gamma^{g \rightarrow g^{*}}\left(\bar{r}, \bar{\Omega} \rightarrow \bar{\Omega}^{-}\right)$and can be written as

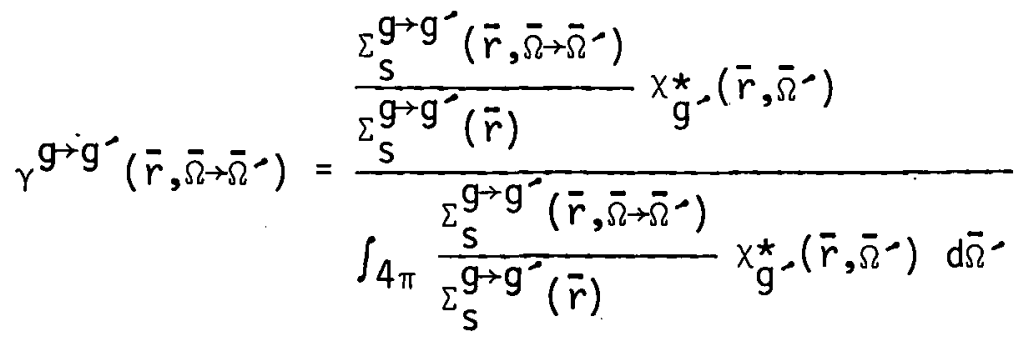

$$
\begin{aligned}
& =\frac{\frac{1}{2 \pi} \sum_{i=1}^{N} p_{i} \delta\left(\mu-\mu_{i}\right) x_{g^{-}}^{\star}\left(\bar{r}, \bar{\Omega}_{-}^{-}\right)}{\int_{4 . \pi} \frac{1}{2 \pi} \sum_{i=1}^{N} p_{i} \delta\left(\mu-\mu_{j}\right) x_{g}^{\star},\left(\bar{r}, \bar{\Omega}^{-}\right) d \bar{\Omega}^{-}} .
\end{aligned}
$$

In Equation (3-10), the $N$ possible outgoing directions $\bar{\Omega}^{-}{ }_{j}\left(\mu_{i}, \phi_{j}\right)$ must be known so that the corresponding point values $x_{g^{-}}^{*}\left(\bar{r}, \bar{\Omega}^{-}{ }_{j}\right)$ can be identified. In order to accomplish this, the azimuthal angles for each of the $\mathrm{N}$ polar angles are selected first. With the $\mathrm{N}$ possible outgoing directions determined, the corresponding point values $\chi_{g}^{\star},\left(\bar{r}, \bar{\Omega}_{i}\right)$ can be identified. This approach of predetermining the $N$ outgoing directions effectively transforms the distribution given by Equation (3-10) into 


$$
\begin{aligned}
& \gamma^{g \rightarrow g^{-}}\left(\bar{r}, \bar{\Omega}_{\rightarrow \rightarrow \bar{\Omega}^{-}}\right)=\frac{\sum_{i=1}^{N} p_{i} \delta\left(\mu-\mu_{i}\right) \delta\left(\phi-\phi_{i}\right) x_{g^{*}}^{*}\left(\bar{r}, \bar{\Omega}^{-}\right)}{\int_{4 \pi} \sum_{i=1}^{N} p_{i} \delta\left(\mu-\mu_{i}\right) \delta\left(\phi-\phi_{i}\right) x_{g^{-}}^{*}\left(\bar{r}, \bar{\Omega}^{-}\right) d \bar{\Omega}^{-}} \\
& =\frac{\sum_{i=1}^{N} p_{i} \delta\left(\Omega^{-}-\Omega^{-}{ }_{i}\right) x_{g^{-}}^{*}\left(\bar{r}, \bar{\Omega}^{-}\right)}{\int_{4 \pi} \sum_{i=1}^{N} p_{i^{\delta}} \delta\left(\bar{\Omega}^{-}-\bar{\Omega}^{-}{ }_{i}\right) x_{g^{-}}^{*}\left(\bar{r}, \bar{\Omega}^{-}\right) \mathrm{d} \bar{\Omega}^{-}} \\
& =\frac{p_{j} x_{g}^{*},\left(\bar{r}, \bar{\Omega}_{j}^{-}\right)}{\sum_{i=1}^{N} p_{i} x_{g}^{*}-\left(\bar{r}, \bar{\Omega}^{-}{ }_{i}\right)} \quad \text { for } i=1,2, \ldots . ., N \\
& \equiv p_{i}^{*} \quad \text { for } i=1,2, \ldots, N \text {. }
\end{aligned}
$$

As would be the case for any biasing procedure, the statistical weight of the particle must be corrected in order to remove the bias. The weight correction is

$$
W T_{C}=\frac{\sum_{i=1}^{N} p_{i} x_{g^{-}}^{*}\left(\bar{r}, \bar{\Omega}_{i}^{-}\right)}{x_{g^{-}}^{*}\left(\bar{r}, \bar{\Omega}^{-}{ }_{j}\right)},
$$

where the direction " $\mathrm{j}$ " has been sclected from the biased distribution given by Equation (3-11).

It is interesting to note that this biasing technique alters the probabilities of the $N$ discrete scattering angles while the corresponding $\mathrm{N}$ possible scattering angles remain unchanged. This is the reason that the name "angular probability biasing" is adopted. 


\subsection{The Path Length Biasing Technique}

The transport of radiation particles from one collision site to the next involves the selection of a path length from the transport kernel. For particles traveling within a material, the transport kernel $T_{g}(\bar{r} \rightarrow \bar{r}+R \bar{\Omega})$ can be written as $(14)$

$$
T_{g}(\bar{r} \rightarrow \bar{r}+R \bar{\Omega}) d R=\Sigma_{t}^{g} e^{-\Sigma_{t}^{g} R} d R,
$$

where $e^{-\Sigma_{t}^{g} R}$ is the probability that a particle travels the distance $R$ without suffering a collision and $\Sigma_{t}^{g} d R$ is the probability that a collision occurs within $d R$. Also since

$$
\int_{0}^{\infty} \Sigma_{t}^{g} e^{-\Sigma_{t}^{g} R} d R=1
$$

the transport kernel $T_{g}(\bar{r} \rightarrow \bar{r}+R \bar{\Omega})$ is a properly normalized p.d.f. The transport kernel can also be expressed in terms of the mean free path $n$, and we have

$$
T_{g}(\bar{r} \rightarrow \bar{r}+R \bar{s}) d R=e^{-n} d n \text {, }
$$

where $n=\Sigma_{t}^{g} R$. Thus, the path length selection can also be accomplished in terms of the number of mean free paths traveled. Equation (3-14) is also valid for particle transport through more than one material. Note that $e^{-n}$ is a normalized p.d.f. over the interval $(0, \infty)$. However, events occur only in the interval $\left(0, n_{e}\right)$, where $n_{e}$ is the total number of mean free paths to the external boundary. When a path is selected from $e^{-n}$, only the 
fraction $\left(1-e^{-n} e\right)$ are events, and the fraction $e^{-n} e$ leak into the external void. Therefore, the leakage probability of a particle is $e^{-n} e$, and the nonleakage probability is $\left(1-e^{-n} e\right)$.

The "path length biasing" technique is an alteration of the transport kernel using the event value $W_{g}(\bar{r}, \bar{\Omega})$ as the importance function. The biased transport kernel $T_{g}^{\star}(\bar{r} \rightarrow \bar{r}+R \bar{\Omega})$ can be defined as

$$
T_{g}^{*}(\bar{r} \rightarrow \bar{r}+R \bar{\Omega}) d R=\frac{e^{-n} W(n)}{N F} d n
$$

where $W(n)$ is the event value with the position expressed in terms of $n$ ( $g$ and $\bar{\Omega}$ are omitted to simplify the notation), and NF is the normalization factor of the biased transport kernel. If the nonleakage probability of the biased transport kernel is assumed to be equal to that of the unbiased transport kernel, we have

$$
\int_{0}^{R} e_{g}^{t}(\bar{r} \rightarrow \bar{r}+R \bar{\Omega}) d R=\int_{0}^{R} e T_{g}(\bar{r} \rightarrow \bar{r}+R \bar{\Omega}) d R
$$

where $R_{e}=n_{e} / \Sigma_{t}^{g}$. Substituting Equations (3-14) and (3-15) into the above equation, we have

$$
\int_{0}^{n} e \frac{e^{-n} W(n)}{N F} d n=\int_{0}^{n} e e^{-n} d n=1-e^{-n} e
$$

Hence,

$$
N F=\frac{1}{1-e^{-n_{e}}} \int_{0}^{n_{e}} e^{-n} W(n) d n .
$$

If an average $W(n)$ is assigned to each spatial region, the event value is given by 


$$
\begin{array}{cc}
W(n)=W_{1} & \text { for } 0 \leq n<n_{1} \\
=W_{2} & n_{1} \leq n<n_{2} \\
\vdots & : \\
=W_{L} & n_{L-1} \leq n<n_{L},
\end{array}
$$

where $L$ is the number of regions along the particle's trajectory and $n_{j}$ is the number of mean free paths from the particle's starting point to the emergent boundary of the $i^{\text {th }}$ region. Note that $n_{L}=n_{e}$. Figure 5 shows a particle's flight trajectory through three regions before reaching the external void. Although the number of regions $L$ would be different for each flight trajectory, $L=.3$ is used to illustrate the analysis which would be valid for any L. Substituting Equation (3-17) with $L=3$ into Equation (3-16), the normalization factor becomes

$$
\begin{gathered}
N F=\frac{1}{i-e^{-n_{3}}}\left[\int_{0}^{n_{1}} e^{-n} w_{1} d_{1}+\int_{n_{1}}^{n_{2}} e^{-n} w_{2} d_{n}+\right. \\
\int_{n_{2}}^{n_{3}} e^{\left.-n w_{3} d_{n}\right] .}
\end{gathered}
$$

Substituting Equation (3-17) into Equation (3-15), the biased transport kernel becomes 


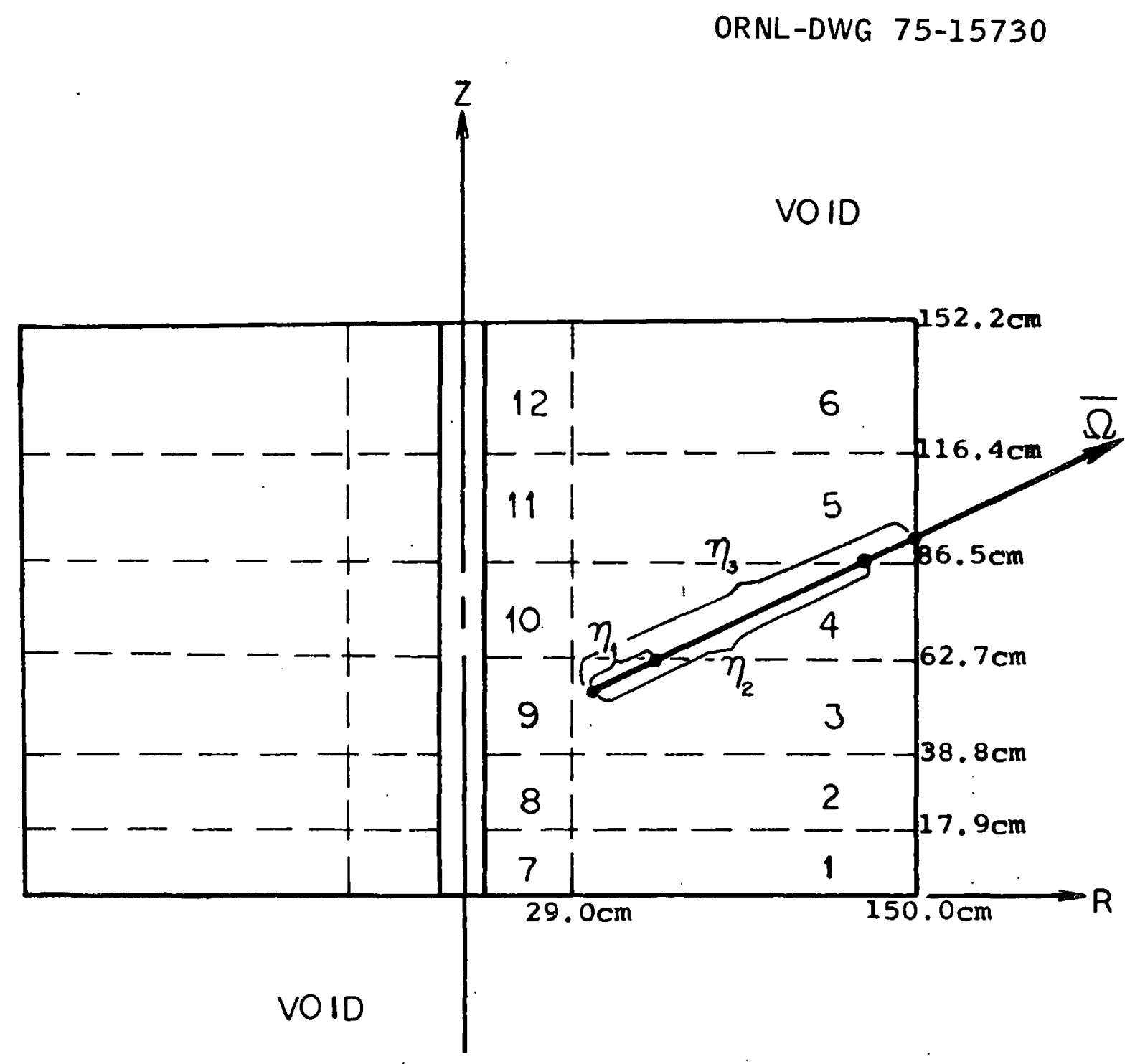

Figure 5. A Typical Particle Flight Trajectory in the Cylindrical Geometry which is Divided into Twelve Spatial Regions. 


$$
\begin{array}{rlrl}
T_{g}^{*}(\bar{r} \rightarrow \bar{r}+R \bar{\Omega}) d R & =\frac{e^{-n} W_{1}}{N F} d n \text { for } & 0 \leq n<n_{1} \\
& =\frac{e^{-n} W_{2}}{N F} d n & & n_{1} \leq n<n_{2} \\
& =\frac{e^{-n} W_{3}}{N F} d n & & n_{2} \leq n<n_{3} \\
& =e^{-n_{d n}} & & n_{3} \leq n<\infty .
\end{array}
$$

The integration of Equation (3-19) over the interval $(0, \infty)$ gives

$$
\begin{gathered}
\int_{0}^{\infty} T_{g}^{*}(\bar{r} \rightarrow \bar{r}+R \bar{\Omega}) d R=\int_{0}^{n_{1}} \frac{e^{-n} w_{1}}{N F} d n+\int_{n_{1}}^{n_{2}} \frac{e^{-n} w_{2}}{N F} d n \int_{n_{2}}^{n_{3}} \frac{e^{-n} w_{3}}{N F} d n+ \\
\int_{n_{3}}^{\infty} e^{-n} d n \\
=\frac{1}{N F}\left[\int_{0}^{n_{1}} e^{-n} w_{1} d n+\int_{n_{1}}^{n_{2}} e^{-n} w_{2} d_{n}+\int_{n_{2}}^{n_{3}} e^{-n} w_{3} d_{n}\right]+e^{-n_{3}} .
\end{gathered}
$$

Substituting Equation (3-18) into Equation (3-20), we have

$$
\int_{0}^{\infty} T_{g}^{*}(\bar{r} \rightarrow \bar{r}+R \bar{\Omega}) d R=1
$$

which is the normalization requirement for the biased transport kernel. With the normalization factor NF given by Equation (3-18), the biased transport kernel is well defined and properly normalized. Hence, Equation (3-20) becomes 


$$
\begin{aligned}
1 & =\frac{W_{1}}{N F} \int_{0}^{\eta_{1}} e^{-n} d \eta+\frac{W_{2}}{N F} \int_{\eta_{1}}^{n_{2}} e^{-n} d n+\frac{W_{3}}{N F} \int_{n_{2}}^{n_{3}} e^{-n} d n+e^{-n_{3}} \\
& =P r_{1}+P r_{2}+P r_{3}+P r_{e},
\end{aligned}
$$

where $\mathrm{Pr}_{j}$ is the probability that the next collision will occur in the ith region and $\mathrm{Pr}_{\mathrm{e}}$ is the probability that the next collision will be in the external void. In principle, the path length can be selected from Equation (3-19). However, the actual sampling procedure is accomplished as follows:

1. First determine the region where the next collision will occur from Equation (3-22).

2. If the next collision is in region " $i$," select the number of mean free paths $n$ the particle travels in this region from

$$
\frac{e^{-n}}{\int_{n_{i-1}}^{n_{i}} e^{-n} d n}=\frac{e^{-\left(n-n_{i-1}\right)}}{1-e^{-\left(n_{i}-n_{i-1}\right)}} .
$$

3. Compute the number of mean free paths between the new collision site and the last collision site by $n_{t}=\eta_{j-1}+n$.

4. Correct the particle's statistical weight by

$$
W T_{c}=\frac{e^{-n}}{\frac{W_{j}}{N F} \int_{n_{j-1}}^{n_{i}} e^{-n} d n \times \frac{e^{-n}}{\int_{n_{j-1}}^{n_{i}} e^{-n} d n}}=\frac{N F}{w_{i}} .
$$




\section{CHAPTER IV}

\section{DESCRIPTION OF THE STANDARD PROBLEM AND GENERATION OF IMPORTANCE FUNCTIONS}

In this chapter, the standard problem and the two-dimensional discrete ordinates adjoint calculations are described. Then the methods of obtaining the point value and the event value from the adjoint discrete ordinates (DOT) calculation are described.

\subsection{Description of the Standard Problem}

The standard problem was designed to have geometric complexity along with deep penetration limitations. The shield configuration consists of a right circular concrete cylinder with an axial duct (void) 15.24 centimeters ( 6 inches) in diameter. The dimensions of the cylinder are 152.2 centimeters in height and 150.0 centimeters in radius. An isotropic monoenergetic (14 MeV) neutron source is uniformly distributed over the bottom surface, and four detectors are positioned on a plane which is 152.2 centimeters beyond the top surface. The radial positions of the detectors are $5,20,75$, and 150 centimeters from the Z-axis. The layout and important dimensions are shown in Figure 6 . One of the main reasons for selecting a two-dimensional configuration was the availability of a discrete ordinates solution which would provide a comparison for Monte Carlo calculations.

Analysis is done only on the neutron transport through the first 14 energy groups of 22 group structure given in Table 1 . The 
ORNL-DWG 75-15727

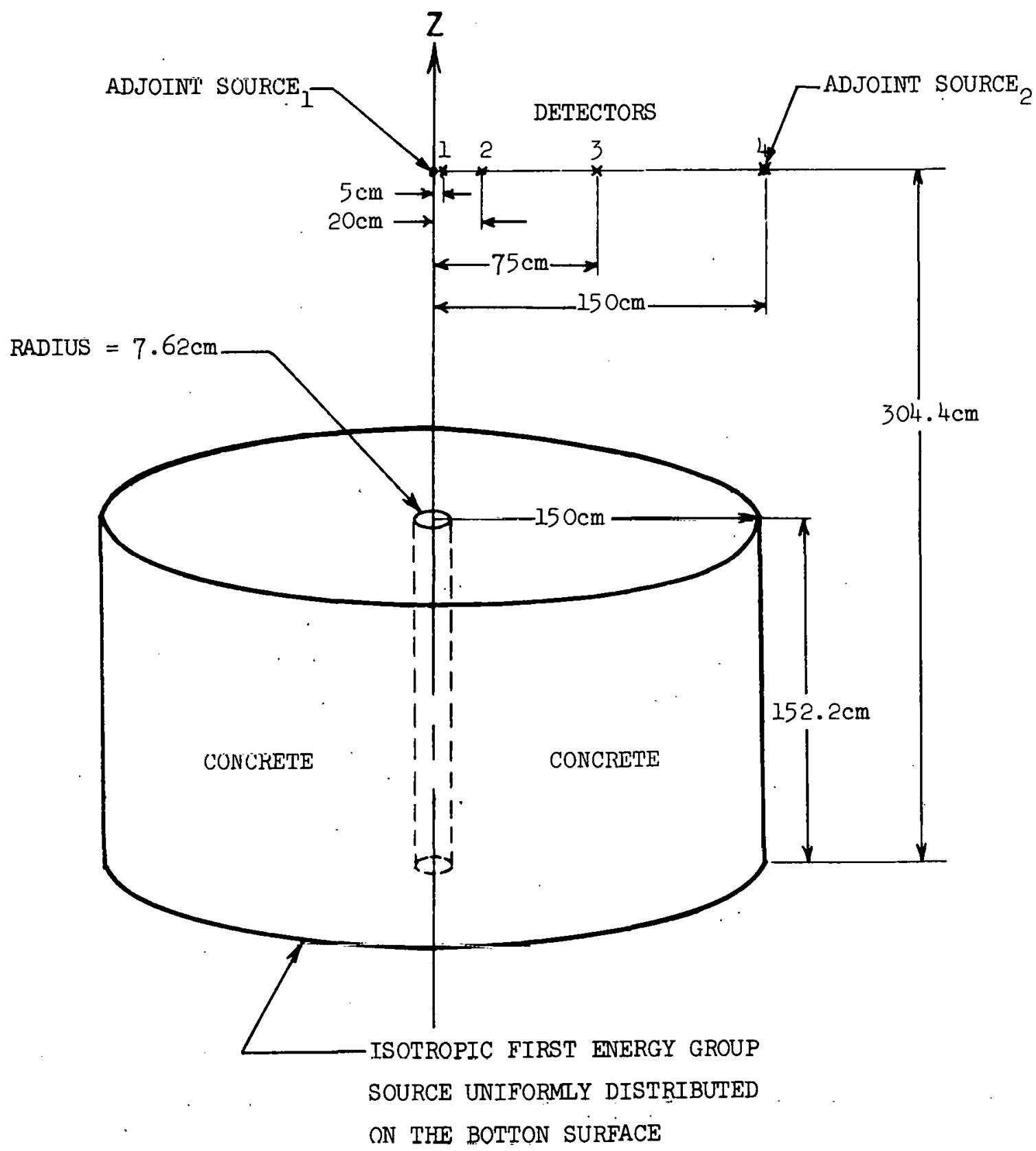

Figure 6. Geometry of Concrete Cylinder with Axial Duct, Source, Detectors, and Adjoint Sources. 
TABLE 1

NEUTRON ENERGY GROUP STRUCTURE

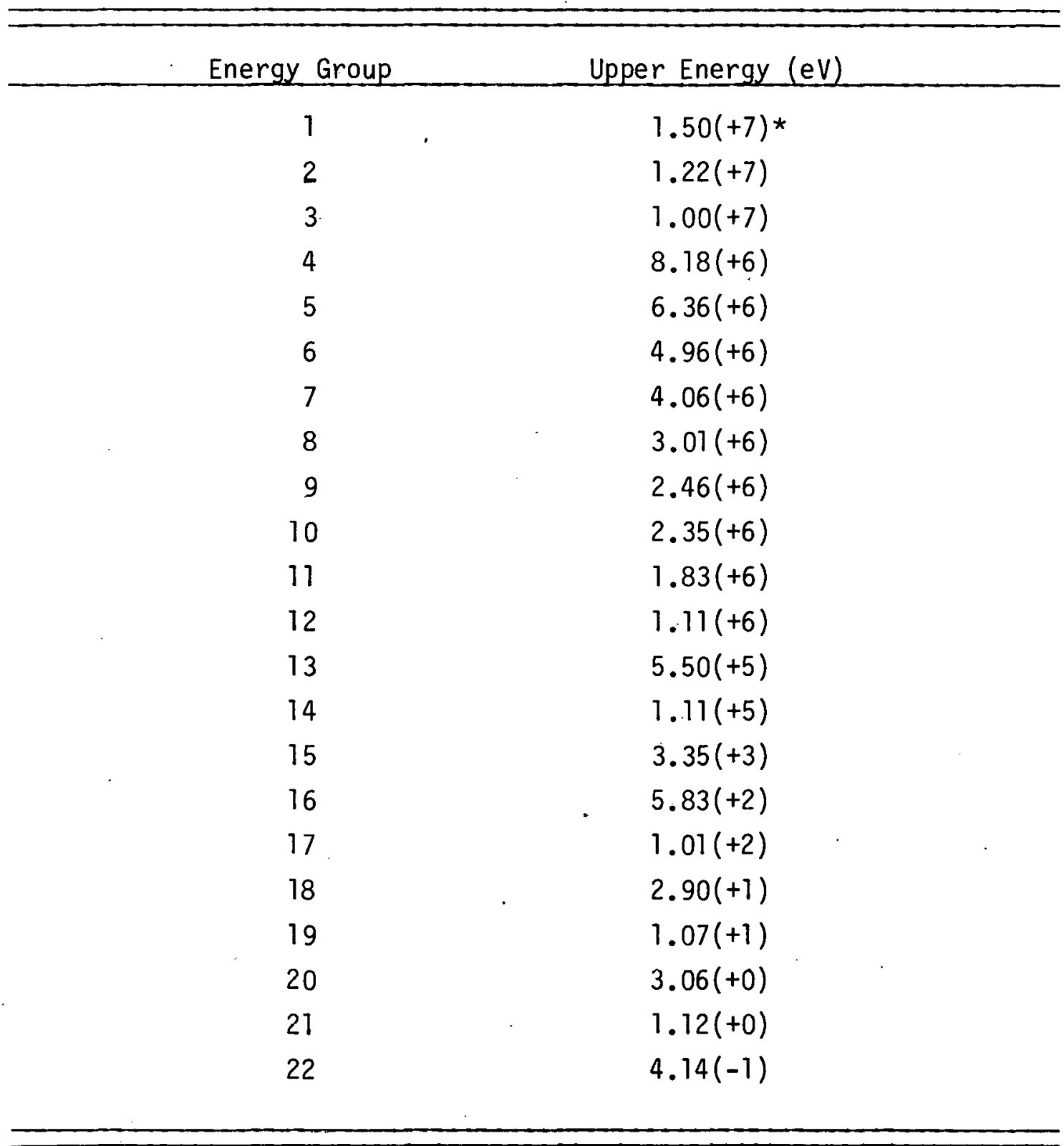

$\star$ Read as $1.50 \times 10^{7} \mathrm{eV}(=15 \mathrm{MeV})$ 
cross section set for the concrete is taken from References 15 and 16; the angular dependence of particle scattering is approximated by a $\mathrm{P}_{3}$ Legendre polynomial series expansion. The concrete composition and its neutron cross section are presented in Appendix $E$.

\subsection{Two-Dimensional Discrete Ordinates Adjoint Calculations}

Several methods have been employed to obtain importance functions for biasing Monte Carlo Calculations. (4-9) Among these methods, the one-dimensional and two-dimensional discrete ordinates calculations provide numerical importance functions suitable for Monte Carlo biasing. For problems with complex geometries, the one-dimensional discrete ordinates adjoint fluxes are generaliy inadequate and two-dimensional adjoint calculations are required. In this study, two-dimensional discrete ordinates calculations are employed to generate the importance functions.

The two-dimensional discrete ordinates code $\mathrm{DOT}{ }^{(11)}$ in $\mathrm{R}-\mathrm{Z}$ geometry was used to solve the adjoint Boltzmann transport equation. An assymmetric angular quadrature set which consists of 166 directions was recuminended for this problem. (25) The source was the detector response function which was taken as unity for all energy group since neutron flux would be the quantity determined by the Monte Carlo calculations. Calculations were performed for two different locations of the adjoint source, one on the Z-axis and the other 150.0 centimeters off the $Z$-axis as shown in Figure 6.

The computer code GRTUNCL (17) was employed to calculate the analytic first collision source for the DOT calculation. The 
adjoint angular flux for each calculation was stored on two magnetic tapes and the total scattering source was written on another magnetic tape.

\subsection{Determination of the Point Value}

It was shown in Section 2.2, page 9, that the point value is identical to the adjoint angular flux which may be calculated by the discrete ordinates method. Hence, the point value is directly available from the results of the adjoint discrete ordinates calculation. Due to the truncated Legendre expansion of the scattering cross section, the adjoint angular flux as calculated by the DOT code may assume negative values. Negative fluxes (forward or adjoint) do not have any physical meaning. Thus, they are assumed to be zero before the adjoint angular flux is spatially averaged into the point value.

The adjoint angular flux is averaged over spatial regions and w1ll be used in that form in the Monte Carlo calculations. This is necessary so as to alleviate the data storage problem that would exist if all of the adjoint angular fluxes were required in the Monte Carlo calculations. Furthermore, it is straightforward to carry out the proposed importance sampling schemes by region in the MORSE $(10)$ code.

The geometry of the concrete cylinder is divided into twelve spatial regions as shown in Figure 5, page 27. The directional dependence of the point value is given in terms of the quadrature set used in the adjoint discrete ordinates calculation. This quadrature set is described in terms of two cosines $\zeta$ and $\varepsilon$, and a weight WG. As 
shown in Figure $7, \zeta$ is the cosine of the polar angle $\gamma$ and $\varepsilon$ is the cosine of $\psi$, the angle between the discrete direction and a unit vector in the radial direction. The weight $W G$ is the fraction of the total $4 \pi$ solid angle associated with a given discrete direction. Thus the weights sum to unity. The discrete directions in the quadrature set are grouped by polar angle and an arbitrary number of $\psi$ angles are associated with each polar angle. For the quadrature set used in the adjoint discrete ordinates calculations, there are 20 polar angles.

The computer program POINT first reads the adjoint angular flux tapes, then sets the negative adjoint angular fluxes to zero, and finally calculates the region-averaged point values for all energy groups and directions. The region-averaged point values are then stored on a magnetic tape for the angular probability biasing of Monte Carlo Calculations. A listing of the POINT program along with the input instruction is presented in Appendix $A$.

\subsection{Determination of the Event Value}

The event value can be determined from the total scattering source that is available from an adjoint DOT calculation. Equation $(2-1)$ can be rewritten as

$$
W_{g}(\bar{r}, \bar{\Omega})=P_{g}^{\psi}(\bar{r}, \bar{\Omega})+\sum_{g^{-}} \int_{4 \pi} \frac{\sum_{s}^{g \rightarrow g^{-}}\left(\bar{r}, \bar{\Omega}^{\prime} \rightarrow \dot{\Omega}^{-}\right)}{\sum_{t}^{g}(\bar{r})} x_{g^{-}}^{*}\left(\vec{r}, \bar{\Omega}^{-}\right) d \bar{\Omega}-.
$$

The point value $x_{g^{*}}^{*}\left(\bar{r}, \bar{\Omega}^{-}\right)$can be written as

$$
x_{g^{-}}^{*}\left(\bar{r}, \bar{\Omega}^{-}\right)=x_{g^{\prime} u^{*}}^{*}\left(\bar{r}, \bar{\Omega}^{-}\right)+x_{g^{\prime} c}^{*}\left(\bar{r}, \bar{\Omega}^{-}\right),
$$


ORNL-DWG 75-15732

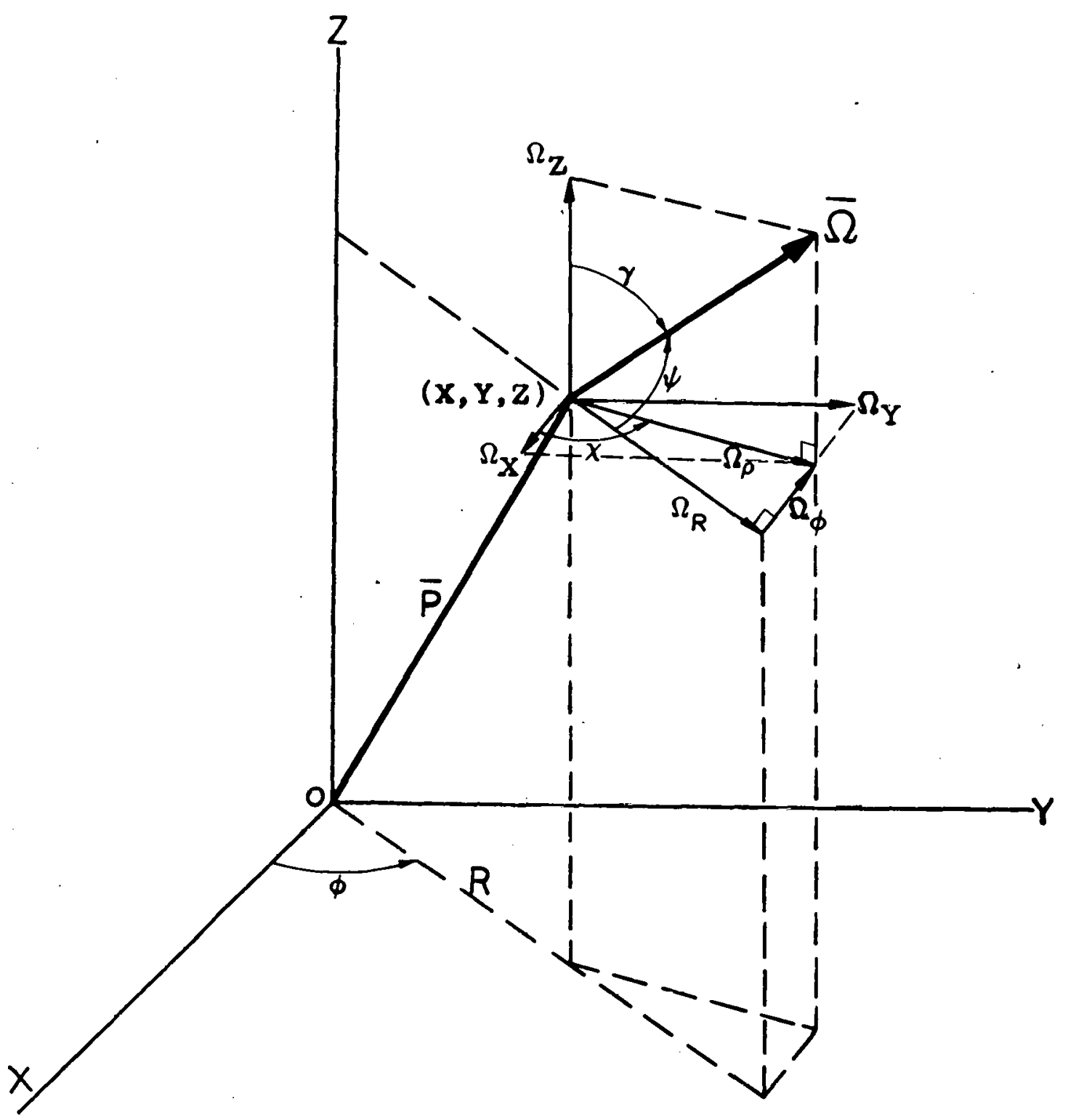

Figure 7. The Components of the Direction Vector $\bar{\Omega}$ in the Rectangular and Cylindrical Coordinate Systems. 
where

$$
\begin{aligned}
& x_{g^{-} u}^{\star}\left(\bar{r}, \bar{\Omega}^{-}\right)=\text {the uncollided contribution to the point value } \\
& x_{g^{-} \mathrm{c}}^{\star}\left(\bar{r}, \bar{\Omega}^{-}\right)=\text {the collided contribution to the point value. }
\end{aligned}
$$

Substituting Equation (4-1) into Equation (2-1), the event value becomes

$$
\begin{aligned}
& W_{g}(\bar{r}, \bar{\Omega})=P_{g}^{\psi}(\bar{r}, \bar{\Omega})+\sum_{g^{-}} \int_{4 \pi} \frac{\Sigma_{s}^{g \rightarrow g^{\prime}}\left(\bar{r}, \bar{\Omega}^{\prime} \rightarrow \bar{\Omega}^{-}\right)}{\sum_{t}^{g}(\bar{r})} x \\
& {\left[x_{g^{-} u}\left(\bar{r}, \bar{\Omega}^{-}\right)+x_{g^{-} c^{*}}\left(\bar{r}, \bar{\Omega}^{-}\right)\right] \mathrm{d} \bar{\Omega}^{-}} \\
& =P_{g}^{\psi}(\bar{r}, \bar{\Omega})+\sum_{g^{\prime}} \int_{4 \pi} \frac{\sum_{s}^{g \rightarrow g^{\prime}}\left(\bar{r}, \bar{\Omega}^{\prime} \rightarrow \bar{\Omega}^{-}\right)}{\sum_{t}^{g}(\bar{r})} x_{g^{-} u^{*}}\left(\bar{r}, \bar{\Omega}^{-}\right) d \bar{\Omega}^{-} \\
& +\sum_{g^{-}} \int_{4 \pi} \frac{\sum_{S}^{g \rightarrow g^{\prime}}\left(\bar{r}, \bar{\Omega}^{-} \rightarrow \bar{\Omega}^{-}\right)}{\sum_{t}^{g}(\bar{r})} x_{g^{-} c}^{*}\left(\bar{r}, \bar{\Omega}^{-}\right) d \bar{\Omega}^{-} \\
& =P_{y}^{\psi}(\bar{r}, \bar{\Omega})+\frac{Q_{g}(\bar{r}, \bar{\Omega})}{\sum_{t}^{g}(\bar{r})}+\sum_{g^{\prime}}, \int_{4 \pi} \frac{\sum_{s}^{g \rightarrow g^{\prime}}\left(\bar{r}, \bar{\Omega}^{\prime} \rightarrow \bar{\Omega}^{-}\right)}{\Sigma_{t}^{g}(\bar{r})} x_{g^{\prime} c^{\star}}\left(\bar{r}, \bar{\Omega}^{-}\right) d \bar{\Omega}^{-},
\end{aligned}
$$

where $Q_{g}(\bar{r}, \bar{\Omega})=\sum_{g^{-}} \int_{4 \pi} \sum_{s}^{g \rightarrow g^{\prime}}\left(\bar{r}, \bar{\Omega}^{\prime} \rightarrow \bar{\Omega}^{-}\right) x_{g^{-} u^{*}}\left(\bar{r}, \bar{\Omega}^{-}\right) d \bar{\Omega}^{-}$.

If the differential scattering cross section from Equation (3-5) is substituted into Equation (4-2), then 


$$
\begin{gathered}
W_{g}(\bar{r}, \bar{\Omega})=P_{g}^{\psi}(\bar{r}, \bar{\Omega})+\frac{Q_{g}(\bar{r}, \bar{\Omega})}{\sum_{t}^{g}(\bar{r})} \\
+\sum_{g^{-}} \int_{4 \pi} \frac{\sum_{\ell^{\prime}=0}^{n} f_{\ell}^{g \rightarrow g^{-}}(\bar{r}) P_{\ell^{\prime}}(\mu)}{4 \pi \sum_{t}^{g}(\bar{r})} x_{g^{\prime} c^{*}}\left(\bar{r}, \bar{\Omega}^{-}\right) d \bar{\Omega}^{-} .
\end{gathered}
$$

The addition theorem of Legendre polynomial can be expressed as (18)

$$
\begin{gathered}
P_{\ell}(\mu)=P_{\ell}(\zeta) P_{\ell}\left(\zeta^{-}\right)+2 \sum_{m=1}^{\ell} \frac{(\ell-m) !}{(\ell+m) !} P_{\ell}^{m}(\zeta) P_{\ell}^{m}\left(\zeta^{-}\right) \cos m\left(\phi-\phi^{-}\right) \\
=P_{\ell}(\zeta) P_{\ell}\left(\zeta^{-}\right)+2 \sum_{m=1}^{\ell} \frac{(\ell-m) !}{(\ell+m) !} P_{\ell}^{m}(\zeta) P_{\ell}^{m}\left(\zeta^{-}\right) x \\
{\left[\operatorname{cosm} \phi \operatorname{cosm} \phi^{-}+\operatorname{sinm} \phi \operatorname{sinm} \phi^{-}\right]}
\end{gathered}
$$

where

$$
\begin{aligned}
P_{\ell}^{m}(\zeta) & =\text { the associated Legendre function } \\
\zeta & =\text { the cosine of the polar angle of the direction } \bar{\Omega} \\
\zeta^{\prime} & =\text { the cosine of the polar angle of the direction } \bar{\Omega}^{-} \\
\phi^{\prime} & =\text { the azimuthal angle of the direction } \bar{\Omega}^{-} \\
\phi^{-} & =\text {the azimuthal angle of the direction } \bar{\Omega}^{-} .
\end{aligned}
$$

Substitution of Equation (4-4) into Equation (4-3) yields the following equation for $w_{g}(\bar{r}, \bar{\Omega})$. 


$$
\begin{aligned}
& W_{g}(\bar{r}, \bar{\Omega})=P_{g}^{\psi}(\bar{r}, \bar{\Omega})+\frac{Q_{g}(\bar{r}, \bar{\Omega})}{\Sigma_{t}^{g}(\bar{r})} \\
& +\frac{1}{4 \pi \Sigma_{t}^{g}(\bar{r})} \sum_{g^{-}} \int_{0}^{2 \pi} \int_{-1}^{l} d \phi^{-} d \zeta^{-} x_{g^{\prime} c}^{*}\left(\bar{r}, \zeta^{-}, \phi^{-}\right) \sum_{l=0}^{n} f_{l}^{g \rightarrow g^{\prime}}(\bar{r}) \\
& x\left\{P_{\ell}(\zeta) P_{\ell}\left(\zeta^{\prime}\right)+2 \sum_{m=1}^{\ell} \frac{(\ell-m) !}{(\ell+m) !} P_{\ell}^{m}(\zeta) P_{\ell}^{m}\left(\zeta^{-}\right) x\right. \\
& \left.\left[\cos m \phi \cos m \phi^{-}+\operatorname{sinm} \phi \operatorname{sinm} \phi^{-}\right]\right\} .
\end{aligned}
$$

Due to the symmetry of cylindrical $R-Z$ geometry, the point value $x_{g^{\prime} C}^{*}\left(\bar{r}, \zeta^{-}, \phi^{-}\right)$is an even function of $\phi^{\prime}$ and can be expanded in spherical harmonics as. a function of $P_{\ell}^{m}\left(\zeta^{\prime}\right)$ cosm $\phi^{\prime}$. Since the integral of $\operatorname{cosm} \phi^{-} \operatorname{sinm} \phi^{\prime}$ over the interval $0 \leq \phi^{\prime} \leq 2 \pi$ is equal to zero, in Equation (4-5), all terms involving sinm $\phi^{-}$varnish under the integration. Thus, Equation (4-5) becomes

$$
\begin{aligned}
& W_{g}(\bar{r}, \bar{\Omega})=P_{g}^{\psi}(\bar{r}, \bar{\Omega})+\frac{Q_{g}(\bar{r}, \bar{\Omega})}{\Sigma_{t}^{g}(\bar{r})}+\frac{1}{4 \pi \Sigma_{t}^{g}(\bar{r})} \sum_{g}-\sum_{l=0}^{n} f_{l}^{g \rightarrow g}(\bar{r}) \\
& x\left[P_{\ell}(\zeta) \int_{0}^{2 \pi} \int_{-1}^{1} d \phi^{-} d \zeta^{-} x_{g^{\prime} c}^{*}\left(\bar{r}, \dot{\zeta}^{-}, \phi^{\prime}\right) P_{\ell}\left(\zeta^{-}\right)\right. \\
& +2 \sum_{m=1}^{\ell} \frac{(\ell-m) !}{(\ell-1 m) !} p_{\ell}^{m}(\zeta) \cos m \phi \int_{0}^{2 \pi} \int_{-1}^{1} d_{\phi^{-}} d \zeta^{\prime} x_{g^{-} c}^{*}\left(\bar{r}, \zeta^{\circ}, \phi^{-}\right) x \\
& \left.P_{\ell}^{m}\left(\zeta^{\prime}\right) \cos m \phi^{-}\right]
\end{aligned}
$$




$$
\begin{gathered}
=P_{g}^{\psi}(\bar{r}, \bar{\Omega})+\frac{Q_{g}(\bar{r}, \bar{\Omega})}{\Sigma_{t}^{g}(\bar{r})}+\frac{1}{4 \pi \Sigma_{t}^{g}(\bar{r})} \sum_{g^{-}} \sum_{\ell=0}^{n} f_{\ell}^{g \rightarrow g^{\prime}}(\bar{r})\left[\sum_{m=0}^{\ell} A_{\ell}^{m}(\zeta, \phi)\right. \\
\left.\quad x \int_{0}^{2 \pi} \int_{-1}^{l} d_{\phi^{\prime}} d \zeta^{-} x_{g^{\prime} c}^{\star}\left(\bar{r}, \zeta^{-}, \phi^{\prime}\right) A_{\ell}^{m}\left(\zeta^{\prime}, \phi^{-}\right)\right]
\end{gathered}
$$

where $A_{\ell}^{m}(\zeta, \phi) \equiv\left[\frac{2}{1+\delta_{o m}} \frac{(l-m) !}{(\ell+m) !}\right]^{1 / 2} P_{\ell}^{m}(\zeta) \cos m \phi$.

In discrete ordinates theory, the integral in Equation (4-6) is known as the flux moment. That is

$$
j_{g^{\prime}}^{l m}(\bar{r}) \equiv \int_{0}^{2 \pi} \int_{-1}^{1} d \phi^{-} d \zeta^{-} x_{g^{-}}^{\star}\left(\bar{r}, \zeta^{-}, \phi^{\prime}\right) A_{\ell^{m}}^{m}\left(\zeta^{-}, \phi^{-}\right) .
$$

With the above relation and setting the response function $\mathrm{P}_{g}^{\psi}(\bar{r}, \bar{\Omega})$ equal to zero because the detectors are located outside the shield configuration, the event value of Equation (4-6) becomes

$$
\begin{gathered}
W_{g}(\bar{r}, \bar{\Omega})=\frac{Q_{g}(\bar{r}, \bar{\Omega})}{\sum_{t}^{g}(\bar{r})} \\
+\frac{1}{4 \pi \Sigma_{t}^{g}(\bar{r})} \sum_{g^{\prime}} \sum_{\ell=0}^{n} \sum_{m=0}^{\ell} f_{\ell}^{g \rightarrow g^{-}}(\bar{r}) A_{\ell}^{m}(\zeta, \phi) j_{g^{\prime}}^{l m}(\bar{r}) .
\end{gathered}
$$

Integration of Equation (4-7) over a volume element $V_{I}$ gives 


$$
\begin{gathered}
\int_{V_{I}} W_{g}(\bar{r}, \bar{\Omega}) d V=\int_{V_{I}} \frac{Q_{g}(\bar{r}, \bar{\Omega})}{\Sigma_{t}^{g}(\bar{r})} d V \\
+\frac{1}{4 \pi} \sum_{g^{\prime}} \sum_{\ell=0}^{n} \sum_{m=0}^{\ell} A_{\ell}^{m}(\zeta, \phi) \int_{V_{I}} \frac{f_{\ell}^{g \rightarrow g^{-}}(\bar{r}) j_{g^{-}}^{\ell m}(\bar{r})}{\Sigma_{t}^{g}(\bar{r})} d V .
\end{gathered}
$$

If the mean value approximation is applied to the volume integral, the above equation becomes

$$
\begin{gathered}
w_{g, I}(\bar{\Omega}) v_{I}=\frac{Q_{g, I}(\bar{\Omega})}{\Sigma_{t, I}^{g}} v_{I} \\
+\frac{1}{4 \pi} \sum_{g^{\prime}} \sum_{\ell=0}^{n} \sum_{m=0}^{\ell} A_{\ell}^{m}(\zeta, \phi) \frac{f_{\ell, I}^{g \rightarrow g^{-}} j_{g^{\prime}, I}^{l m}}{\Sigma_{t, I}^{g}} v_{I}
\end{gathered}
$$

$Q_{g, I}(\bar{\Omega})$ can be expanded in terms of spherical harmonics as

$$
Q_{g, I}(\bar{\Omega})=\sum_{\ell=0}^{n} \sum_{m=0}^{\ell} \frac{2 \ell+1}{4 \pi} q_{g, I}^{\ell m} A_{\ell}^{m}(\zeta, \phi)
$$

where $q_{g, I}^{l m}=\int_{0}^{2 \pi} \int_{-1}^{1} Q_{g, I}(\bar{\Omega}) A_{\ell}^{m}(\zeta, \phi) d \phi d \zeta$.

Substituting Equation (4-9) into Equation (4-8), we have

$$
\begin{aligned}
& W_{g, I}(\bar{s}) V_{I}=\frac{I}{4 \pi \Sigma_{t, I}^{g}} \sum_{\ell=0}^{n} \sum_{m=0}^{\ell} A_{\ell}^{m}(\zeta, \phi) x \\
& {\left[(2 \ell+1) q_{g, I}^{\ell m} V_{I}+\sum_{g^{\prime}} f_{\ell, I}^{g \rightarrow g^{-}} j_{g^{\circ}, I}^{\ell m} V_{I}\right] .}
\end{aligned}
$$


Equation (4-10) can be further simplified by defining the "total scattering source" for energy group $g$ and volume element $V_{I}$ as

$$
T_{g, I}^{\ell m}=(2 \ell+1) q_{q, I}^{\ell m} V_{I}+\sum_{g^{-}} \underset{\ell, I}{g \mapsto g^{-} j_{g^{-}, I}^{\ell m}} V_{I} .
$$

Substitution of Equation (4-11) into Equation (4-10) yields

$$
W_{g, I}(\bar{\Omega}) V_{I}=\frac{1}{4 \pi \Sigma_{t, I}^{g}} \sum_{\ell=0}^{n} \sum_{m=0}^{\ell} A_{l}^{m}(\zeta, \phi) T_{g, I}^{\ell m} .
$$

Equation (4-12) is the relationship actually employed to calculate the event values. Similar to the point value, the event value is averaged over region for all energy groups and directions. A computer program EVENT was written to read the total scattering source tape from the adjoint DOT calculation and to calculate the event values for all regions, energy groups, and directions. A listing of this program along with the input instruction is given in Appendix $A$.

Shown in Figure 8 is a flow diagram which illustrates the relationships which exist among the adjoint DOT calculation, the generation of importance functions by the POINT and EVENT programs, and the MORSE calculation. The source location biasing will be discussed in the next chapter. 
ORNL-DWG 75-15725

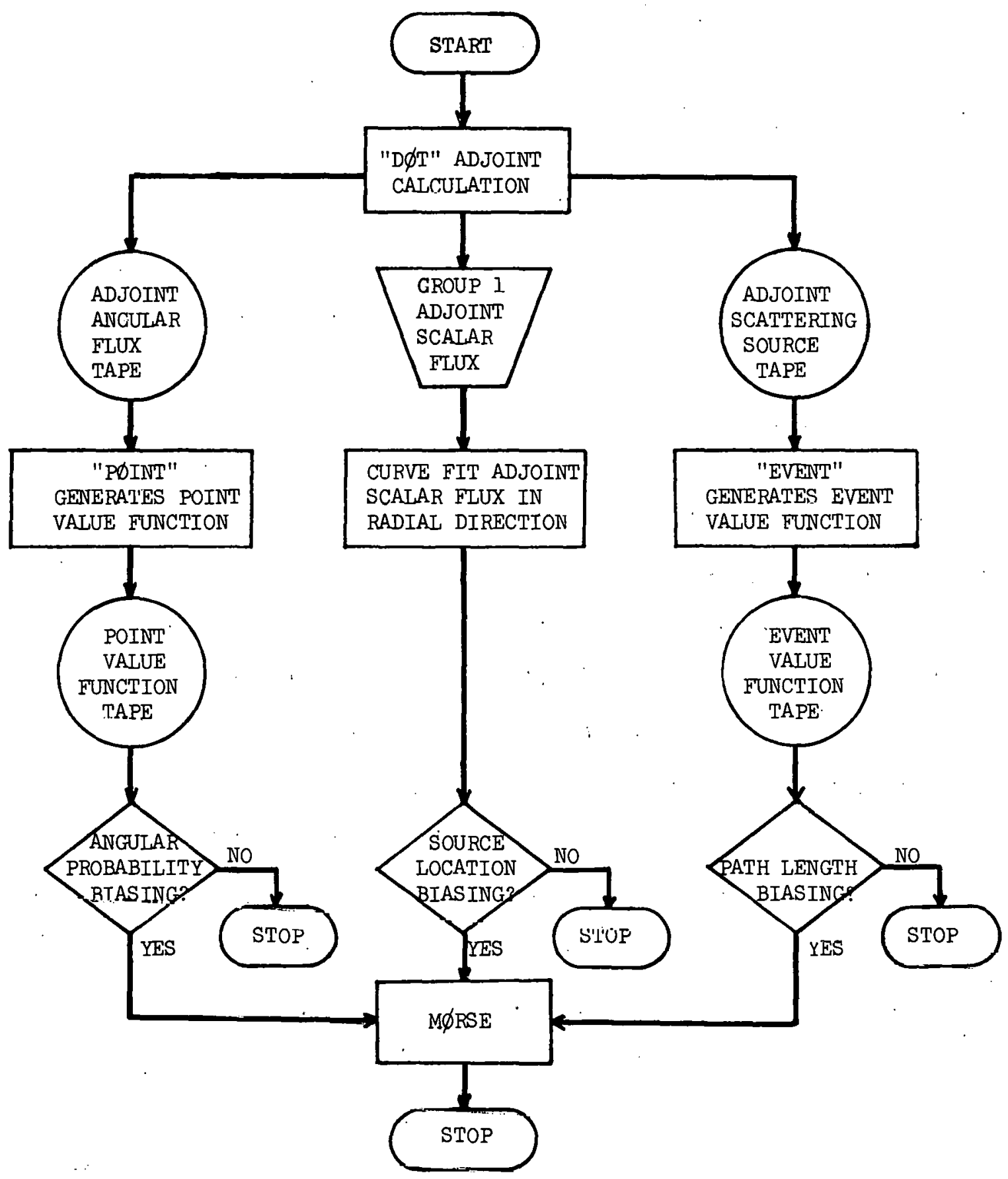

Figure 8. Flow Diagram of Generation of Importance Functions from the Adjoint DOT Calculation and Application to MORSE Calculation. 
CHAPTER V

DESCRIPTION OF THE BIASING TECHNIQUES

The description of the biasing techniques including some programming details will be presented in this chapter. The importance sampling techniques studied in this investigation were source location biasing, path length biasing, and angular probability biasing. In addition, the survival biasing schemes of nonabsorption weighting and Russian roulette and splitting were routinely used in all calculations performed.

\subsection{Source Location Biasing}

It was observed by Coveyou et al. (3) that source biasing using a reasonably good estimate of the importance function would, in general, reduce variance significantly at a relatively low cost. However, a good estimate of the importance function at the source requires that much is known about the solution a priori.

A particle random walk starts with the selection of energy group, spatial position, direction, and age from a source distribution function. For the problem under investigation, the age is assumed to be zero since this is a steady state problem, and the source energy is of the first group. Thus, only the direction and spatial position remain to be selected. Although the source is isotropic, only the upward source particles can contribute to the answer because the downward source particles enter an external void and are immediately killed. Therefore, it is expedient to force all source particles to 
emerge in the upward direction and to correct their weight accordingly. As for the source spatial position, particles which originate near the duct would seem to be more important because they may penetrate deeper into the shield by streaming through the duct. Thus, biasing of the source location to encourage more particles to originate near the duct was carefully studied in this work.

Two importance functions for source location biasing were employed. The first one will be designated as the step importance function which assumes that source particles generated inside the radius of 10 centimeters are 1000 times more important than those outside. This procedure is rather arbitrary, but it does encourage more particles to originate near the duct. The probability density function of this biased source distribution is simply

$$
\begin{array}{rlrl}
\tilde{S}_{1}(R) & =\frac{\frac{2 \pi R}{\pi(150)^{2}}}{N F_{1}}(1000) & & 0 \leq R<10 \\
& =\frac{\frac{2 \pi R}{\pi(150)^{2}}}{N F_{1}} & 10 \leq R \leq 150,
\end{array}
$$

where $N \bar{F}_{1}$ is the normalization factor. The weight correction requires that the statistical weight of particles outside the 10 centimeter radius to be 1000 times higher than that of particles inside.

The second source importance function was obtained from the adjoint DOT calculation. It was assumed that the adjoint source was located on the axis of the cylinder and the corresponding adjoint flux should show the maximum effect of the duct. The group one scalar adjoint flux $x_{j}^{*}$ at the bottom of the cylinder was plotted as a function 
of the radial position. This curve was then fitted by the function $I_{S}(R)$ which is given by Equation (5-2).

$$
\begin{array}{rlrl}
I_{S}(R) & =85 & 0 & \leq R<8 \\
& =e^{-0.13(R-8)} & & \leq R<50 \\
& =0.004 e^{-0.02(R-50)} & & 50 \leq R \leq 150 .
\end{array}
$$

Figure 9 shows the group one scalar adjoint flux and $I_{S}(R)$ versus the radial position. Mathematically, the second biased source distribution takes on the following form

$$
\tilde{S}_{2}(R)=\frac{S(R) I_{S}(R)}{\int_{0}^{T 50} S(R) I_{S}(R) d R} .
$$

Substitution of $S(R)$ and $I_{S}(R)$ into the equation above yields

$$
\begin{array}{rlrl}
\tilde{S}_{2}(R) & =\frac{\frac{2 \pi R}{\pi(150)^{2}}(85)}{\int_{0}^{150} S(R) I_{S}(R) d R} & 0 \leq R<8 \\
& =\frac{\frac{\pi \pi R}{\int_{0}^{150} S(R) I_{S}(R) d R} e^{-0.13(R-8)}}{} \\
& =\frac{2 \pi R}{\int_{0}^{150} S(R) I_{S}(R) d R}(0.004) e^{-0.02(R-50)} & & 50 \leq R \leq 150 .
\end{array}
$$

The integral in the denominator can be easily evaluated. Equation (5-3) can be rewritten as 


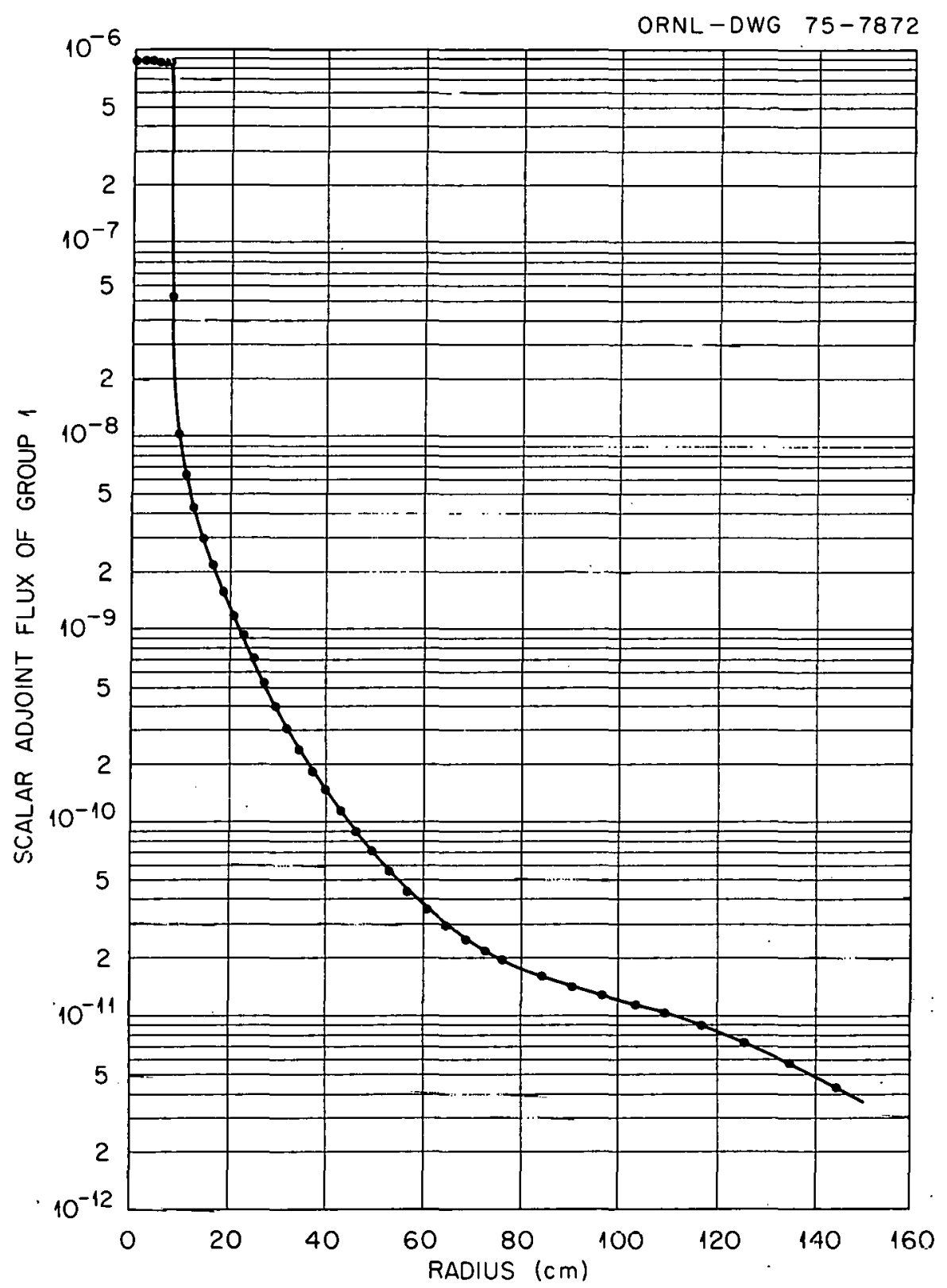

Figure 9. Source Location Importance Function from the Adjoint DOT Calculation with Axial Adjoint Source, and the Approximating Function $I_{s}(R)$ to the Importance Function. 


$$
\begin{array}{rlrl}
\tilde{S}_{2}(R) & =\frac{85 R}{N F_{2}} & & \leq R<8 \\
& =\frac{R e^{-0.13(R-8)}}{N F_{2}} & & 8 \leq R<50 \\
& =\frac{0.004 R e^{-0.02(R-50)}}{N F_{2}} & 50 \leq R \leq 150,
\end{array}
$$

where $N F_{2}=\frac{\int_{0}^{150} S(R) I_{S}(R) d R}{\frac{2 \pi}{\pi(150)^{2}}}$.

In order to remove the bias, the statistical weights of the source particles are corrected by

$$
W T_{c}=\frac{S(R)}{\tilde{S}_{2}(R)} \text {. }
$$

Subroutine SOURCE in the MORSE code is an optional routine which allows a user to generate source particles according to any desircd distribution and to make the necessary weight correction. Two SOURCE routines were employed in this study. One selects source location according to the distribution in Equation (5-1), and the other selects source location from Equation (5-4). Only upward source directions were allowed in both cases. The SOURCE routines are listed in Appendix $A$.

\subsection{Angular Probability Biasing}

The theoretical development of the angular probability biasing technique was discussed in Section 3.2, page 22, and the method of 
determining the point value was presented in Section 4.3, page 34 . This section describes the implementation of this biasing technique in the MORSE code.

The subroutine COLISN in MORSE is called at each collision site to determine the outgoing energy group and the outgoing direction. To carry out the angular probability biasing, the subroutine COLISN was modified and a subroutine INSCOR was written to input the regionaveraged point values from the magnetic tape prepared by the POINT program. In the modified COLISN subroutine, the outgoing energy is still selected from the downscattering matrix, but the outgoing direction is selected from the biased angular distribution given by Equation $(3-11)$

$$
\gamma^{g \rightarrow g^{\prime}}\left(\bar{r}, \bar{\Omega}_{\rightarrow} \bar{\Omega}^{-}\right)=\frac{p_{i} x_{g}^{*}\left(\bar{r}_{,} \bar{\Omega}^{-}{ }_{i}\right)}{\sum_{i=1}^{N} p_{i} x_{g^{-}}^{*}\left(\bar{r}_{,}, \bar{\Omega}^{-}{ }_{i}\right)} \quad \text { for } i=1,
$$

Then the statistical weight of the emergent particle is corrected by Equation (3-12) as shown below.

$$
W T_{c}=\frac{\sum_{j=1}^{N} p_{j} x_{g^{*}}-\left(\bar{r}, \bar{\Omega}_{i}^{-}\right)}{x_{g}^{*}-\left(\bar{r}, \bar{\Omega}_{j}^{-}\right)} \text {. }
$$

The biased angular distribution of Equation (3-11) requires the point values for all outgoing directions which comprise the $\mathrm{N}$ discrete directions of the generalized Gaussian quadrature scheme. The outgoing directions are expressed in terms of the rectangular coordinate system, but the point values are given in terms of the quadrature set used in the $R-Z$ discrete ordinates adjoint calculation. 
Thus, the outgoing directions are transformed into the cylindrical $R-Z$ coordinate system, and the point values that are nearest to each of these directions are chosen. Then the biased angular distribution is constructed according to Equation (3-11).

The transformation of a direction from the rectangular coordinate system to the cylindrical $R-Z$ coordinate system and the reverse transformation are presented in Appendix B. A listing of the subroutines INSCOR and COLISN are presented in Appendix $A$.

\subsection{Path Length Biasing}

The path length biasing technique utilizes the event values to importance sample particle flight paths (i.e., collision sites). The determination of the event values from a two-dimensional discrete ordinates adjoint calculation was discussed in Section 4.4, page 35 . To implement the path length biasing technique in the MORSE code, the subroutine INSCOR was written to read the event values from a magnetic tape, and the subroutine NXTCOL was rewritten to carry out the actual selection of the next collision site.

When a particle emerges from a collision site with a given energy group and direction, its flight trajectory is determined by the subroutine REGION which subsequently returns to NXTCOL the regions that this particle may go through and the corresponding track lengths within those regions. The particle's direction must be transformed into the cylindrical $R-Z$ coordinate system so that the event value corresponding to the direction closest to the particle's direction can be determined for each region. Now that the event values and the track lengths in these regions are known, the selection of the 
path length (or the next collision site) from the biased path length distribution according to Equation (3-19) can be achieved. For easy reference, the biased transport kernel is included here:

$$
\begin{aligned}
T_{g}^{\star}(\bar{r} \rightarrow \bar{r}+R \bar{\Omega}) d R & =\frac{e^{-n} W_{1}}{N F} d n & & \leq n<n_{1} \\
& =\frac{e^{-n_{W}}}{N F} d n & & n_{1} \leq n<n_{2} \\
& =\frac{e^{-n_{1}} W_{3}}{N F} d n & & n_{2} \leq n<n_{3} \\
& =e^{-n} d n & & n_{3} \leq n<\infty .
\end{aligned}
$$

The details of the sampling procedure is outlined in Section 3.3, page 24, and the listings of subroutines INSCOR, NXTCOL, and REGION are included in Appendix A.

Due to the curvature effect in the cylindrical $R-Z$ coordinate system, the angle $\psi$ between the radial vector and the flight direction vector changes as the particle travels through the geometry as illustrated in Figure 10. In this figure, the flight direction vector $\bar{\Omega}$ is assumed to be on the same plane as the radial vector. This curvature effect complicates the determination of the event values, and requires for each region the determination of the direction that is closest to $\bar{\Omega}$. To simplify this procedure, this curvature effect is ignored and the angle $\psi_{1}$ is used for all regions in determining the event values. The error caused by this assumption is not severe because for a given energy group, region, and polar angle, the event value is relatively constant with respect to the angle $\psi$. 
ORNL-DWG 75-15731

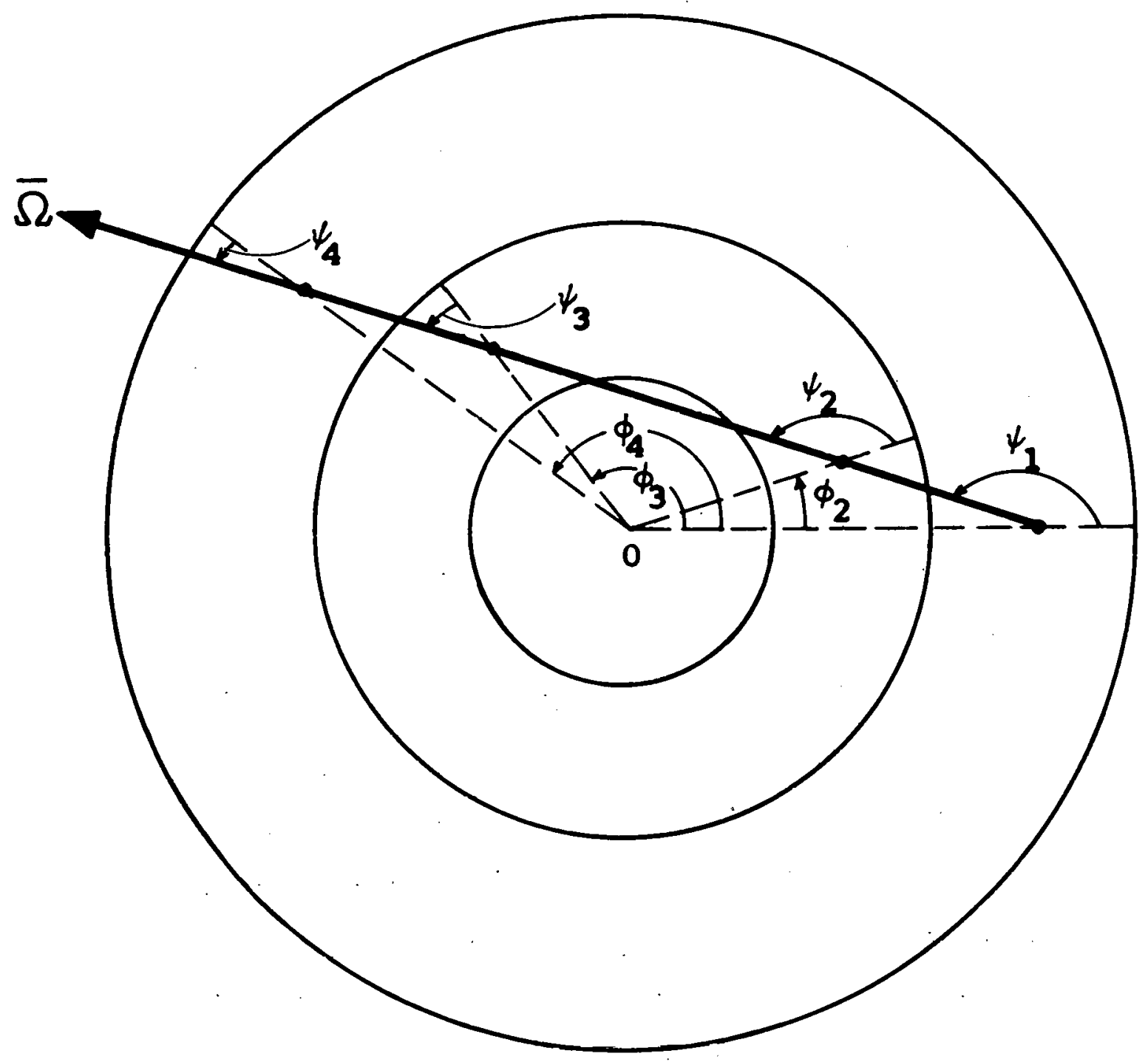

Figure 10. Curvature Effect of a Particle Direction in a Cylindrical Geometry. 


\subsection{Russian Roulette and Splitting}

Russian roulette and splitting is a form of survival biasing. In the MORSE code, Russian roulette and splitting are applied through a set of region and energy-group dependent weight standards. The weight standards are composed of WTHIR, WTLOR, and WTAVE. In order for Russian roulette and splitting to be effective, a good choice of weight standards must be made. For problems involving complex geometry and deep penetration, an effective set of weight standards is not easy to specify. Usually, weight standards are chosen on the basis of intuition, experience, or some knowledge of the problem under investigation.

As pointed out by Solomito, (21) WTAVE in a set of weight standards may be related to the importance function by the following

$$
\lambda=W T(P) \times I(P),
$$

where WT $(P)$ could be used for WTAVE. This equation simply states that the effect of interest $\lambda$ is equal to the weight of a particle WT(P) times its importance $I(P)$ with respect to that effect, and is valid at any point in phase space. Al the sourcc location, $W T(P)=W T_{j}(P)$ and $I(P)=I_{0}(P)$. Hence, the effect of interest $\lambda$ is determined, and it can be used to determine the weight $W T(P)$ at other points provided that the importance $I(P)$ is known. That is

$$
W T(P)=\frac{\lambda}{I(P)}=\frac{W T_{0}(P) \times I_{0}(P)}{I(P)} .
$$


In this study, Russian roulette and splitting were employed routinely in all calculations performed. The same weight standards were routinely used so that the relative merits of the various biasing techniques can be studied. WTAVE was determined using Equation (5-5). The total adjoint flux $x^{*}$ obtained from the DOT discrete ordinates adjoint calculation with the axial adjoint source was taken as the importance function $I(P)$, and an approximate value of the effect of interest at detector 1 was used to calculate WTAVE. Then WTHIR was arbitrarily assumed to be 10 times of WTAVE, and WTLOR was assumed to be 40 times lower than WTAVE. 
CHAPTER VI

\section{DISCUSSION OF RESULTS}

Presented in this chapter are the results of the Monte Carlo calculations of the standard problem. The proposed methods of source biasing, of angular probability biasing, and of path length biasing were thoroughly studied using a modified version of the Monte Carlo code MORSE. The modifications allowed the biasing techniques to be incorporated into the random walk subroutines COLISN and NXTCOL. Source biasing, angular probability biasing, and path length biasing were employed individually and in various combinations. The list of calculations which were performed to test the proposed biasing techniques is presented in Table 2. The importance functions used in these calculations were obtained from the adjoint DOT calculation with the axial adjoint source. Russian roulette and splitting were employed in all calculations. The weight standards for calculations $(0.5)^{*}$ and (SS) - were intuitively assigned, but the same weight standards were. employed for the rest of the calculations (S) through (SAP) and were obtained from the adjoint flux of the DOT calculation with the axial adjoint source.

In order to facilitate the evaluation of results, all calculations were performed for one hour on the Oak Ridge National Laboratory IBM $360 / 91$ computer and the size of a batch of particles

*For easy reference, each calculation will be identified by the naming given in the parenthesis. 
TABLE 2

MONTE CARLO CALCULATIONS PERFORMED

Calculation

Identification

Biasing Procedures Employed

$(0.5)$

(A)

(P)

(AP)

$(S P)$

Standard calculation with path length stretching parameter $\mathrm{PATH}=0.5$

Step biasing of source location and PATH $=0.5$

Source location biasing according to the scalar adjoint flux and $\mathrm{PATH}=0.5$

Angular probability biasing according to the point value $x^{*}$ with step biasing of source location and $\mathrm{PATH}=0.5$

Path length biasing according to the event value $W$ and step biasing of source location

Combination of angular probability biasing, path length biasing, and step biasing of source

location

Combination of source location biasing by the scalar adjoint flux and path length biasing by $w$

Combination of source location biasing by the scalar adjoint flux and angular probability biasing by $x *$ with PATH $=0.5$

Combination of source location biasing, angular probability biasing, and path length biasing 
was standardized to 400 histories. Neutron flux at four detector locations were calculated using the next event estimator at each collision and source point.

\subsection{Comparison of Results of Monte Carlo and Discrete Ordinates Calculations}

Tables 3 and 4 present the uncollided flux and the total flux respectively at the four detector locations for the nine different biasing schemes. The total flux as calculated by the forward discrete ordinates (DOT) calculation is also included in Table 4 for comparison. It is clear from Table 3 that the uncollided flux could be calculated rather easily. Source location biasing using either the step importance function or the scalar adjoint flux generally gives better results for the uncollided flux. However, the (0.5) calculation which has no source biasing gives the best results for detectors 3 and 4 because the natural source distribution produces a large fraction of source particles far away from the duct. A final point of interest about the uncollided flux is that it constitutes $82 \%$ of the total flux at detector 1 and $47 \%$ at detector 2 . For detectors 3 and 4 , the uncollided flux contributes very little to the total flux and the presence of the duct probably has little effect on the results of the uncollided flux.

According to Table 4, Monte Carlo calculations are in general agreement with the DOT calculation. However, the Monte Carlo results are higher by $10 \%$ to $16 \%$ at detectors 1 and 2 , and lower by $20 \%$ to $40 \%$ at detectors 3 and 4 except for the $(0.5)$ and $(P)$ calculations, 
TABLE 3

UNCOLLIDED NEUTRON FLUX ${ }^{\mathrm{a}}$ OF MONTE: CARLO CALCULATIONS OF THE STANDARD PROBLEM

\begin{tabular}{|c|c|c|c|c|c|c|}
\hline $\begin{array}{l}\text { Calculation } \\
\text { Identification }\end{array}$ & $\begin{array}{c}\text { Number } \\
\text { of } \\
\text { Batches }\end{array}$ & & 1 & 2 & ors: & 4 \\
\hline$(0.5)$ & 31 & & $2.431-9(.139)^{b}$ & $3.971-10(.315)$ & $4.764-15(.079)$ & $6.488-16(.012)$ \\
\hline (SS) & 57 & & $2.327:-9(.007)$ & $5.153-10(.018)$ & $5.577-15(.107)$ & $6.640-16(.024)$ \\
\hline$(S)$ & 60 & & $2.348-9(.004)$ & $5.086-10(.031)$ & $5.579-15(.097)$ & $7.751-16(.199)$ \\
\hline (A) & 60 & & $2.352-9(.006)$ & $5.212-10(.018)$ & $5.230-15(.116)$ & $6.334-16(.028)$ \\
\hline$(P)$ & 41 & & $2.350-9(.007)$ & $5.151-10(.016)$ & $6.617-15(.137)$ & $6.685-16(.044)$ \\
\hline$(A P)$ & 49 & & $2.356-9(.007)$ & $5.252-10(.022)$ & $6.456-15(.118)$ & $6.616-16(.036)$ \\
\hline$(S P)$ & 54 & $:$ & $2.347-9(.004)$ & $5.209-10(.031)$ & $4.997-15(.067)$ & $5.775-16(.195)$ \\
\hline$(S A)$ & 72 & & $2.345-9(. \mathrm{CO} 3)$ & $5.093-10(.023)$ & $5.211-15(.070)$ & $6.714-16(.174)$ \\
\hline (SAP) & .62 & & $2.337-9(.003)$ & $5.351-10(.021)$ & $5.942-15(.072)$ & $6.348-16(.150)$ \\
\hline
\end{tabular}

$a_{\text {Unit }}=$ neutrons $/ \mathrm{cm}^{2} /$ source neutron.

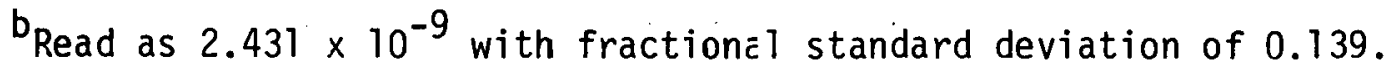


TABLE 4

TOTAL NEUTRON FLUX $x^{\mathrm{a}}$ OF MONTE CARLO CALCULATIONS OF THE STANDARD PROBLEM

\begin{tabular}{cccccc}
\hline $\begin{array}{c}\text { Calculation } \\
\text { Identification }\end{array}$ & $\begin{array}{c}\text { Number } \\
\text { of } \\
\text { Batches }\end{array}$ & 1 & \multicolumn{3}{c}{ Detectors: } \\
\hline (0.5) & 31 & $2.868-9(.124)^{\mathrm{b}}$ & $9.071-10(.159)$ & $4.677-11(.498)$ & $2.241-11(.472)$ \\
$(\mathrm{SS})$ & 57 & $3.092-9(.048)$ & $1.195-9(.149)$ & $2.907-11(.269)$ & $1.062-11(.425)$ \\
$(\mathrm{S})$ & 60 & $3.025-9(.026)$ & $1.158-9(.073)$ & $2.831-11(.173)$ & $1.160-11(.211)$ \\
(A) & 60 & $2.928-9(.024)$ & $1.085-9(.077)$ & $3.039-11(.157)$ & $1.165-11(.188)$ \\
(P) & 41 & $2.830-9(.028)$ & $1.036-9(.051)$ & $3.987-11(.237)$ & $1.746-11(.397)$ \\
(AP) & 49 & $2.911-9(.024)$ & $1.113-9(.067)$ & $3.357-11(.223)$ & $1.170-11(.255)$ \\
(SP) & 54 & $2.805-9(.020)$ & $1.020-9(.084)$ & $3.002-11(.175)$ & $1.136-11(.227)$ \\
(SA) & 72 & $2.839-9(.014)$ & $1.058-9(.045)$ & $3.459-11(.243)$ & $1.261-11(.269)$ \\
(SAP) & 62 & $2.873-9(.023)$ & $1.120-9(.030)$ & $2.764-11(.186)$ & $1.030-11(.222)$ \\
& & & & & \\
DOT & & $2.453-9$ & $9.575-10$ & $3.703-11$ & $1.408-11$.
\end{tabular}

$a_{\text {Unit }}=$ neutrons $/ \mathrm{cm}^{2} /$ source neutron.

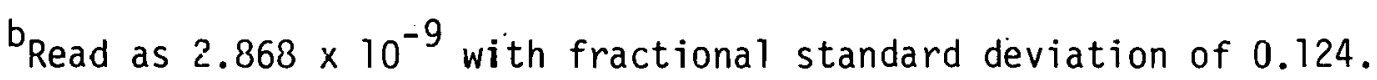


Because of the excellent statistics and the consistency of the Monte Carlo results for detectors 1 and 2 , it would not be unreasonable to presume that MORSE's answers are correct and that the discrete ordinates results are too low. It should be noted that this is somewhat characteristic for the discrete ordinates solution to problems of this type. In the discrete ordinates calculation, the neutron dose for detectors located in an external void can be calculated by the procedure of next event estimation. In this procedure, the probable uncollided contribution to the detector weighted by the neutron flux is integrated over all collision sites.

This integration is accomplished by the computer code FALSTF. (23) For the standard problem, the major contribution to the neutron flux at detectors 1 and 2 is from the neutron distribution near the duct. If the spatial mesh around the duct was too coarse, the flux at detectors 1 and 2 would probably be underestimated due to the increased attenuation to the detectors from the centrnids of the spatial cells. This is the probable explanation for the characteristically lower magnitudes of the DOT's results at detectors 1 and 2 .

The discrepancy between Monte Carlo and discrete ordinates results at detecturs 3 and 4 might have been due to: (1) the statistical fluctuation in Monte Carlo calculations, (2) underestimation which is a common occurrence in Monte Carlo solutions to deep penetration problems. 


\subsection{Comparison of Fractional Standard Deviations}

The fractional standard deviations (FSD) of the total neutron flux from the Monte Carlo calculations with different biasing techniques are presented in Table 5. The results are arranged according to the decreasing order of the FSD's associated with detector 1. Essentially, the same decreasing order is true for the other detectors as well. Individually, detector 1 receives the most benefit from the biasing techniques followed in order by detectors 2, 3, and 4. The FSD decreases from $12 \%$ to about $2 \%$ for detector 1 , from $16 \%$ to about $7 \%$ for detector 2, from $50 \%$ to less than $20 \%$ for detector 3 , and from $47 \%$ to $20 \%$ for detector 4 .

It is apparent, from Table 5, that the (0.5) calculation is grossly inadequate. Source biasing by the step function (SS) improves the FSD's for detectors 1 and 3 a great deal but not much help for other two detectors. Using the importance functions $x^{\star}$ and $W$ obtained from the adjoint DOT calculation with axial adjoint source, the $(S),(A)$, and $(P)$ calculations significantly reduce the FSD's for all four detectors. It is interesting to note that the improved source biasing $(S)$ reduces the FSD at each of the four detectors by one-half: The combinations of any two (of the three) biasing techniques, i.e., (AP), (SP), or (SA), show some improvement over $(S),(P)$, and $(A)$. However, there seems to be a general tendency of these calculations to underestimate the answers. Finally, no further gain is obtained from the combination of all three biasing techniques (SAP) and the answer itself is strongly underestimated. 
TABLE 5

FRACTIONAL STANDARD DEVIATIONS OF THE TOTAL NEUTRON FLUX FROM THE MONTE CARLO CALCULATIONS WITH VARIOUS SAMPLING TECHNIQUES FOR THE STANDARD PROBLEM

\begin{tabular}{ccccc}
\hline & & \multicolumn{3}{c}{ Detectors: } \\
$\begin{array}{c}\text { Calculation } \\
\text { Identification }\end{array}$ & 1 & 2 & 3 & 4 \\
\hline (0.5) & 0.124 & 0.159 & 0.498 & 0.472 \\
(SS) & 0.048 & 0.149 & 0.269 & 0.425 \\
(P) & 0.028 & 0.051 & 0.237 & 0.397 \\
$(\mathrm{~S})$ & 0.026 & 0.073 & 0.173 & 0.211 \\
(A) & 0.024 & 0.077 & 0.157 & 0.188 \\
(AP) & 0.024 & 0.067 & 0.223 & 0.255 \\
(SAP) & 0.023 & 0.080 & 0.186 & 0.222 \\
(SP) & 0.020 & 0.084 & 0.175 & 0.227 \\
(SA) & 0.014 & 0.045 & 0.243 & 0.269 \\
\hline
\end{tabular}


An important point not to be overlooked is that the three biasing schemes, $(S),(P)$, and $(A)$, each utilized (or represented) the importance data to different degrees of precision. The source biasing ( $S$ ) most completely utilized the importance data because the scalar adjoint flux could be quite accurateiy approximated by the analytical function $I_{S}(R)$ which was the importance function for source biasing $(S)$. The path length biasing and the angular probability biasing techniques utilized the adjoint information in a less precise manner because the importance functions used in these biasing schemes were region-averaged quantities.

\subsection{Spatial Distribution of Collision Sites}

Figures 11 through 16 show the spatial distribution of neutron collision sites according to regions within the concrete shield. For the (0.5) calculation, Figure 11 shows that a large fraction of collisions occurred in regions far removed from the duct. This is largely due to the unbiased source distribution which generated most of the source particles away from the duct. The step biasing of the source and the source biasing by DOT scalar adjoint flux greatly increased the source particles produced near the duct. Hence, the percent of collisions occurring within regions 7 through 12 increased considerably. The angular probability biasing and the path length biasing seemed to encourage the particles to penetrate deeper into the shield as can be seen in Figures 13, 14, and 16 . 


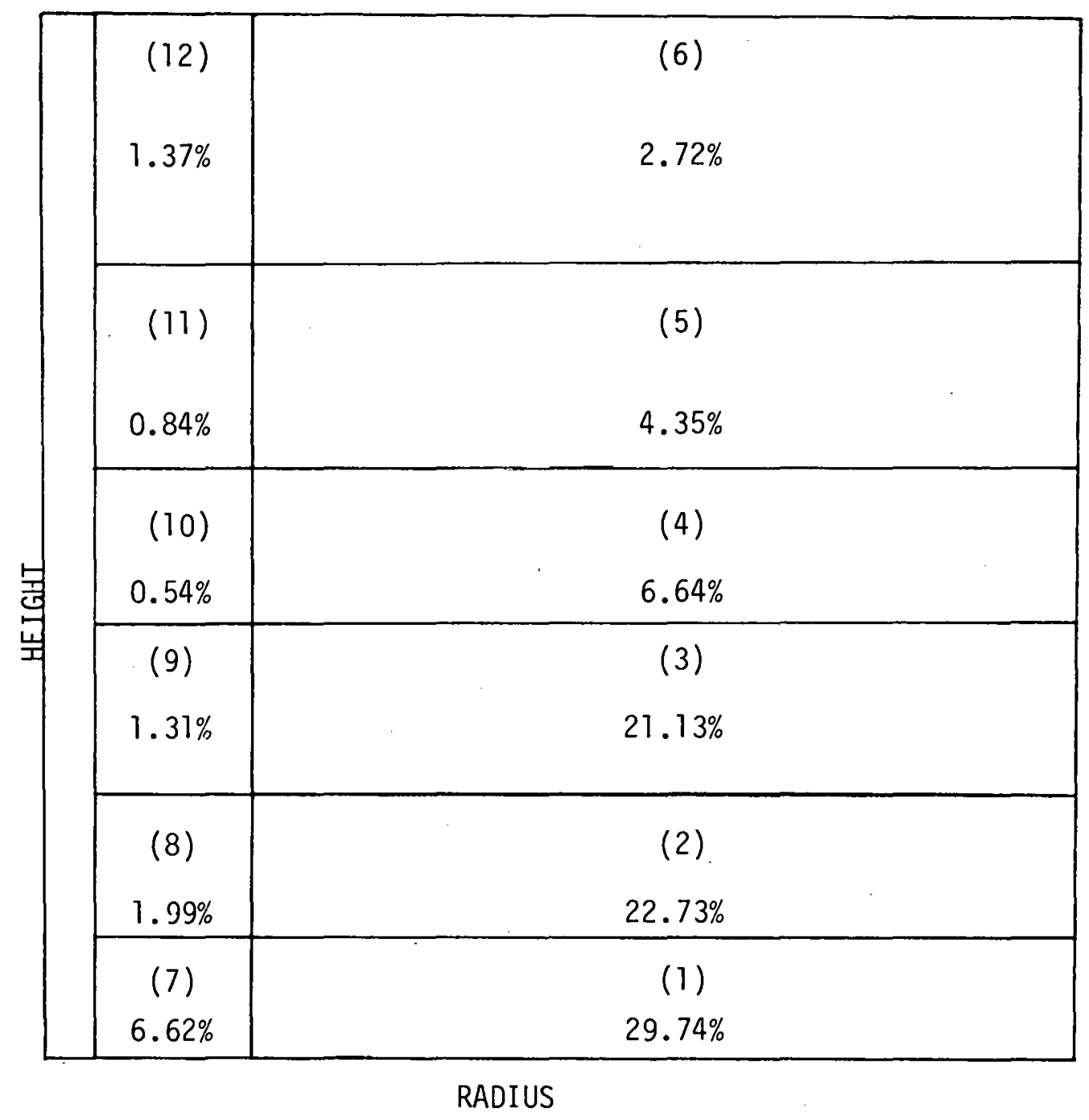

Figure 11. Distribution of Neutron Collision Sites for the (0.5) Calculation. 


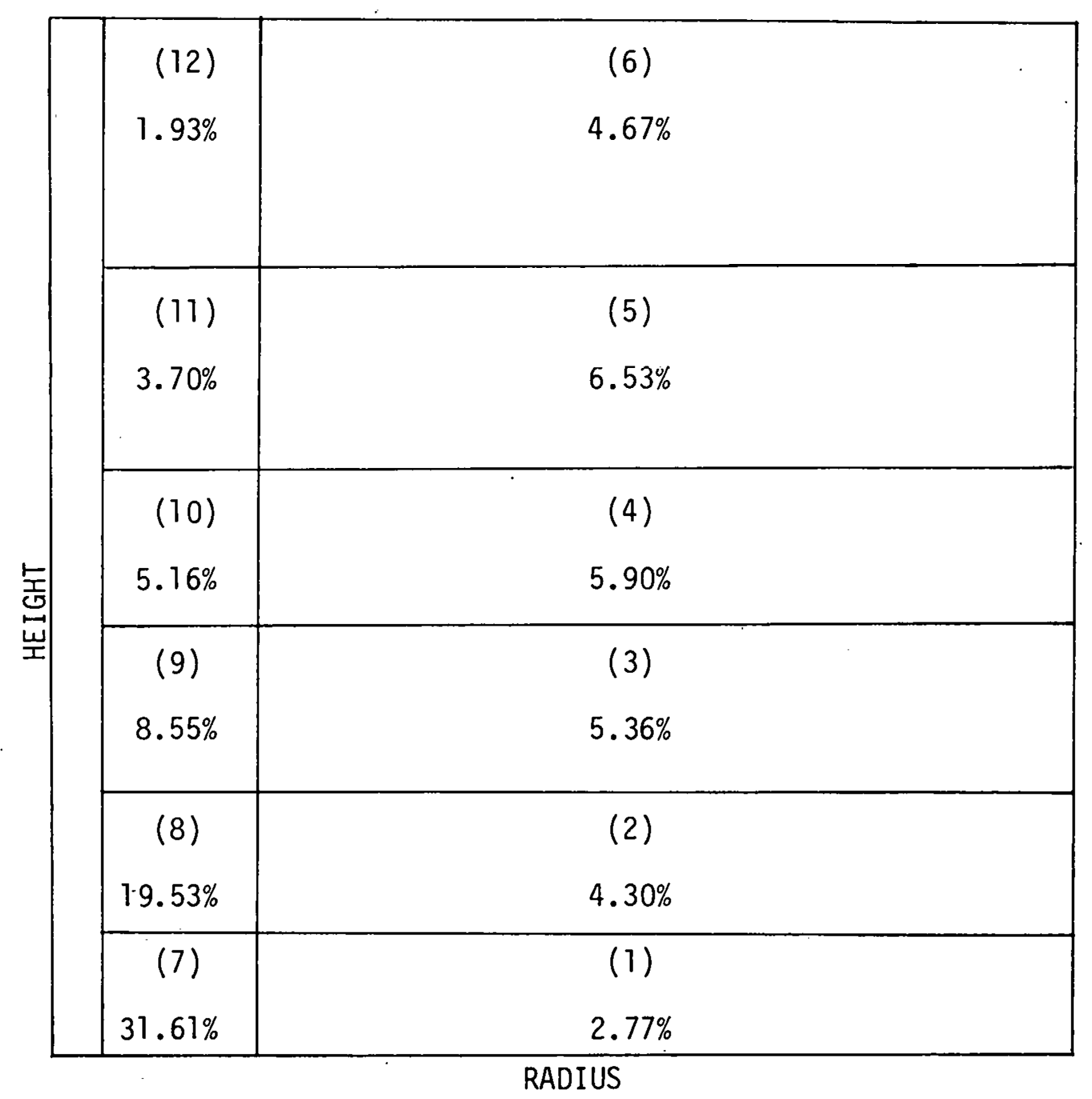

Figure 12. Distribution of Neutron Collision Sites for the (S) Calculation. 


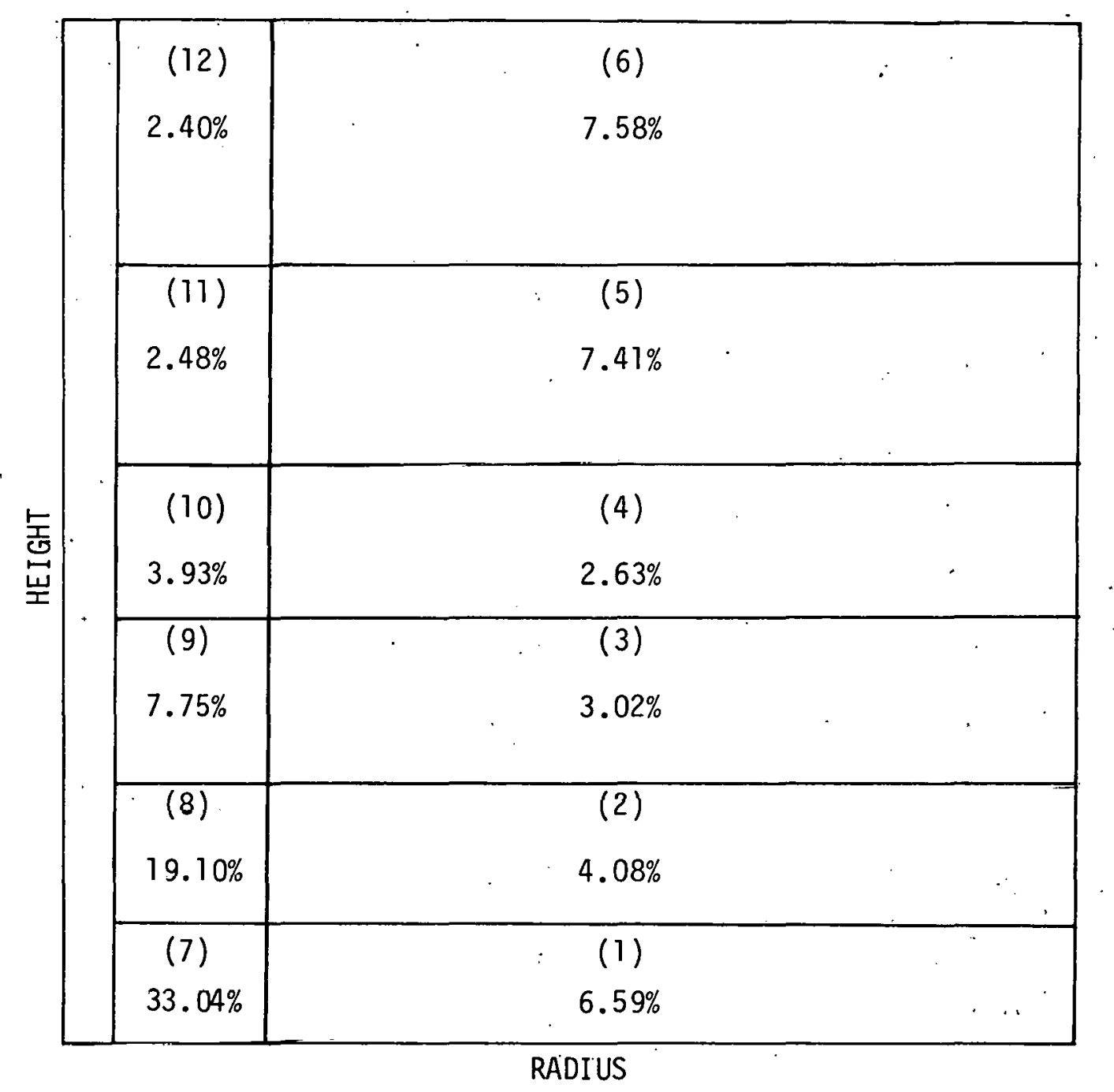

Figure 13. Distribution of Neutron Collision Sites for the (P) Calculation. 


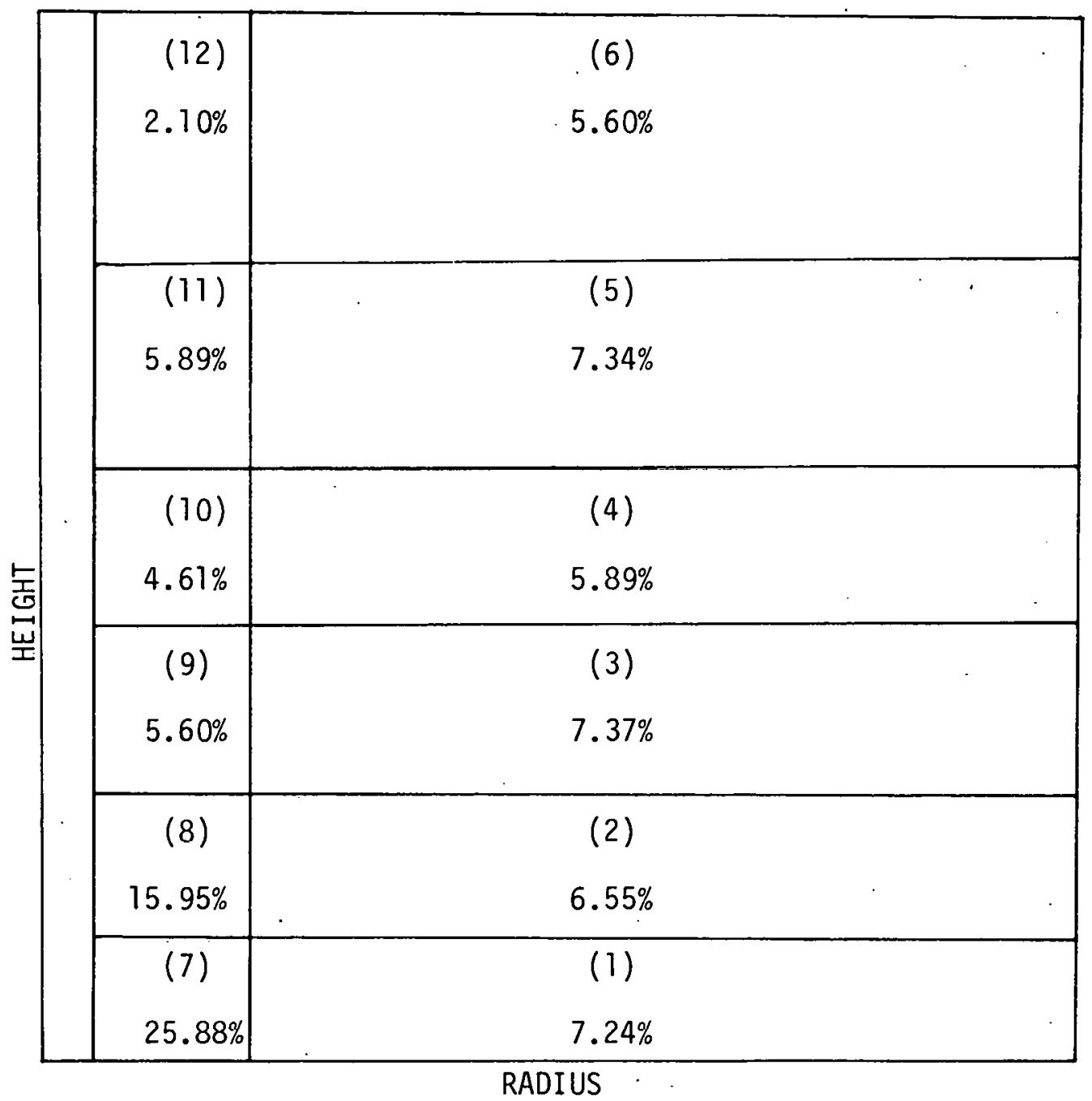

Figure 14. Distribution of Neutron Collision Sites for the (A) Calculation. 


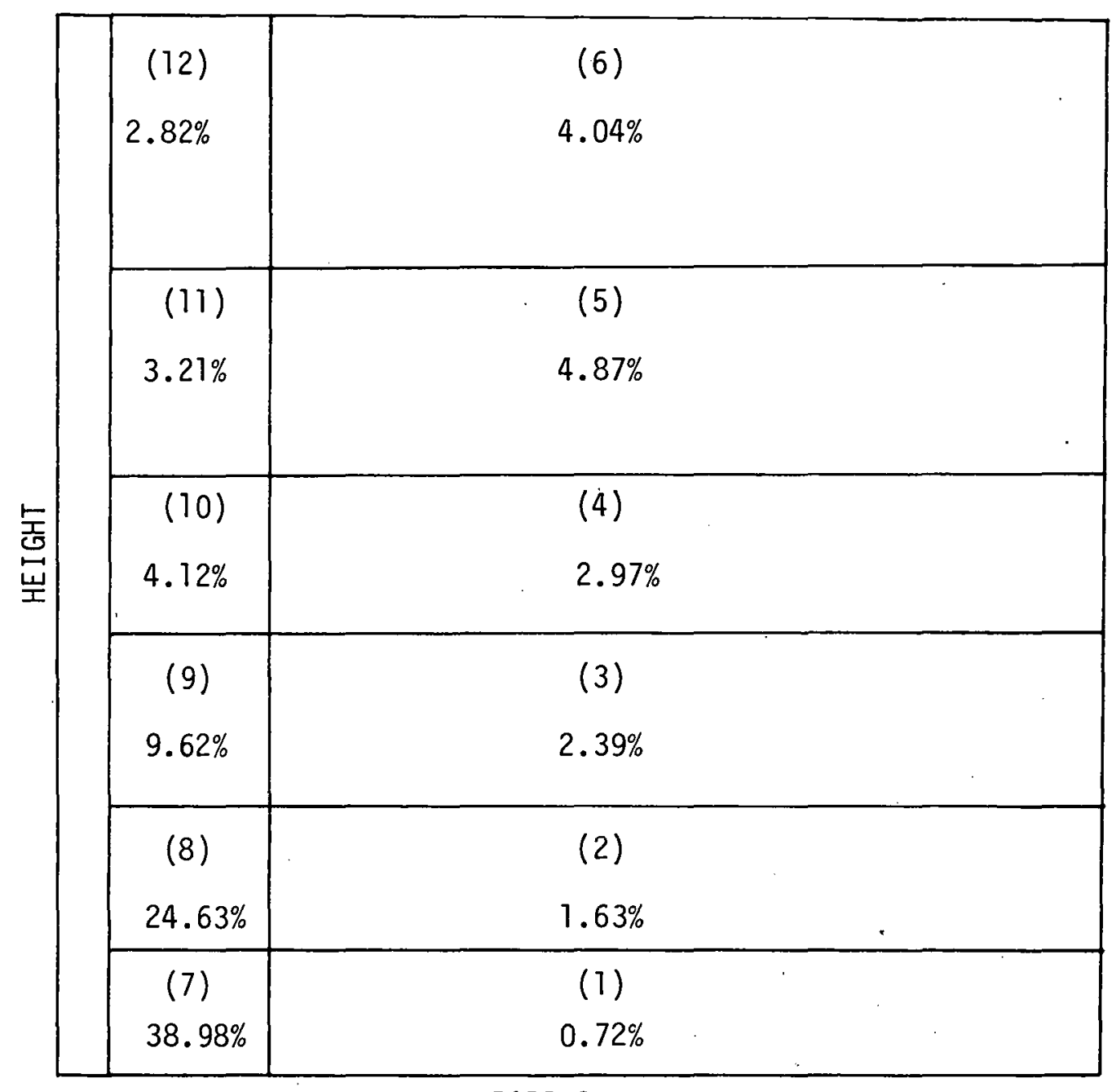

RADIUS

Figure 15. Distribution of Neutron Collision Sites for the (SP) Calculation. 


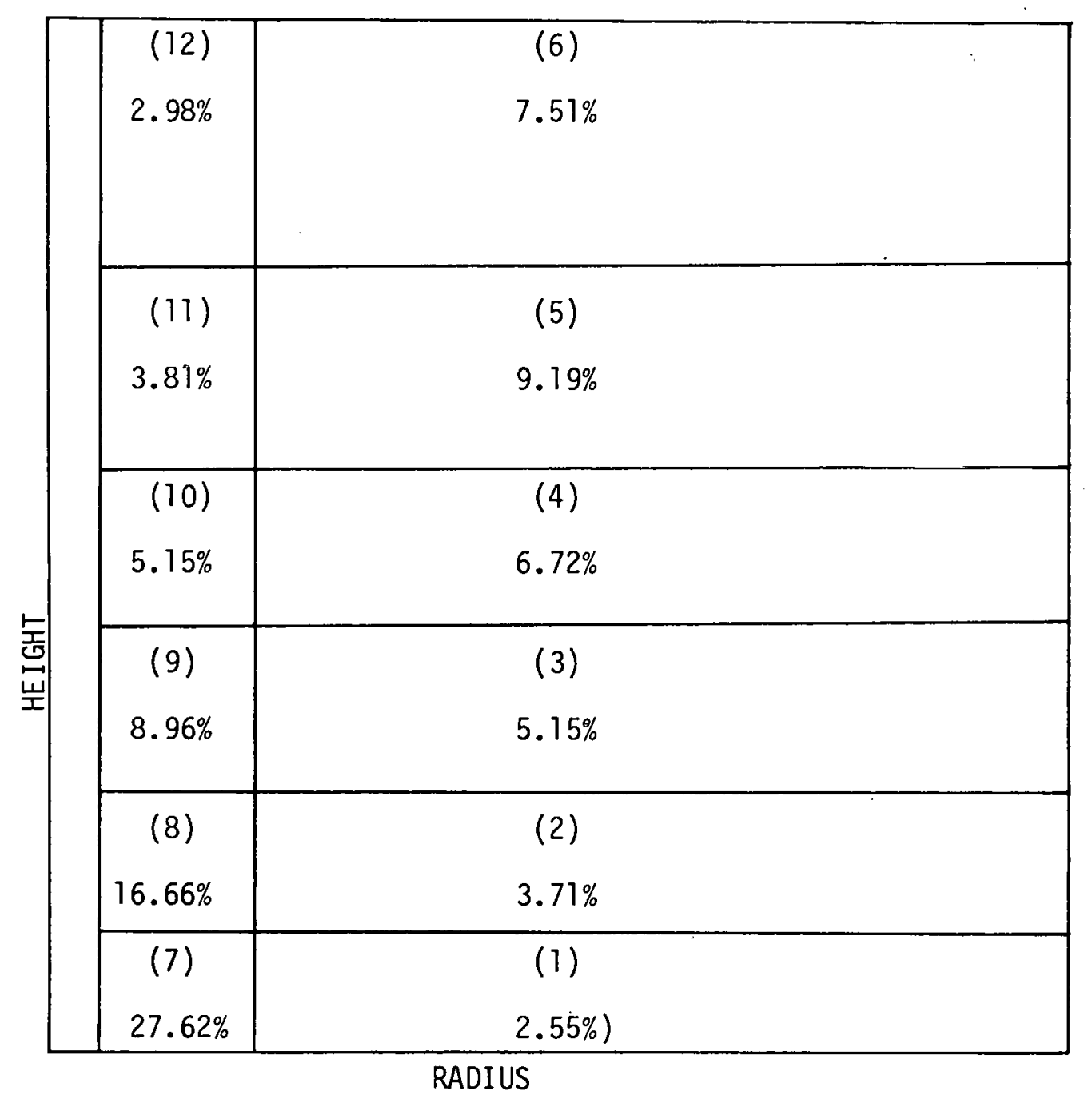

Figure 16. Distribution of Neutron Collision Sites for the (SA) Calculation. 


\subsection{Path Length Biasing Using Different Importance Functions}

Tables 6 and 7 compare results of Monte Carlo calculations using different importance functions for path length biasing and angular probability biasing. Each calculation consumed one hour computer time, and the batch size was 400 histories.

Table 6 is a comparison of results of Monte Carlo calculations using different importance functions for the path length biasing. The $(0.5)$ calculation is also. included because it represented the currently accepted practice. The event value and the point value were employed as the importance functions in $(P)$ and $\left(P^{-}\right)$respectively. These value functions were obtained from the same adjoint DOT calculation with axial adjoint source. However, the event value used in $(P-)$ was obtained from the adjoint DOT calculation with the off-axial adjoint source. Obviously, the $(0.5)$ calculation is grossly ineffective. Among the other three calculations, $\left(P^{-}\right)$has the highest FSD's for detectors 1 and 2, and the lowest FSD's for detectors 3 and 4 . Basen on the results of detectors 1 and 2 , the event value would seem to be the appropriate importance function for biasing the transport kernel.

\subsection{Angular Probability Biasing Using Different Point Values}

Table 7 contains a comparison of results for the (0.5) calculation and for the angular probability biasing using point values obtained from adjoint DOT calculations with the axial and the off-axial adjoint sources. The FSD's of $(A)$ and $\left(A^{-}\right)$calculations are very close at all four detectors. For angular probability biasing, the $x^{*}$ importance function obtained from the adjoint DOT calculation with the off-axial adjoint 
TABLE 6

COMPARISON OF TOTAL NEUTRON FLUX ${ }^{\mathrm{a}}$ OF MONTE CARLO CALCULATIONS USING DIFFERENT IMPORTANCE FUNCTIONS FOR PATH LENGTH BIASING

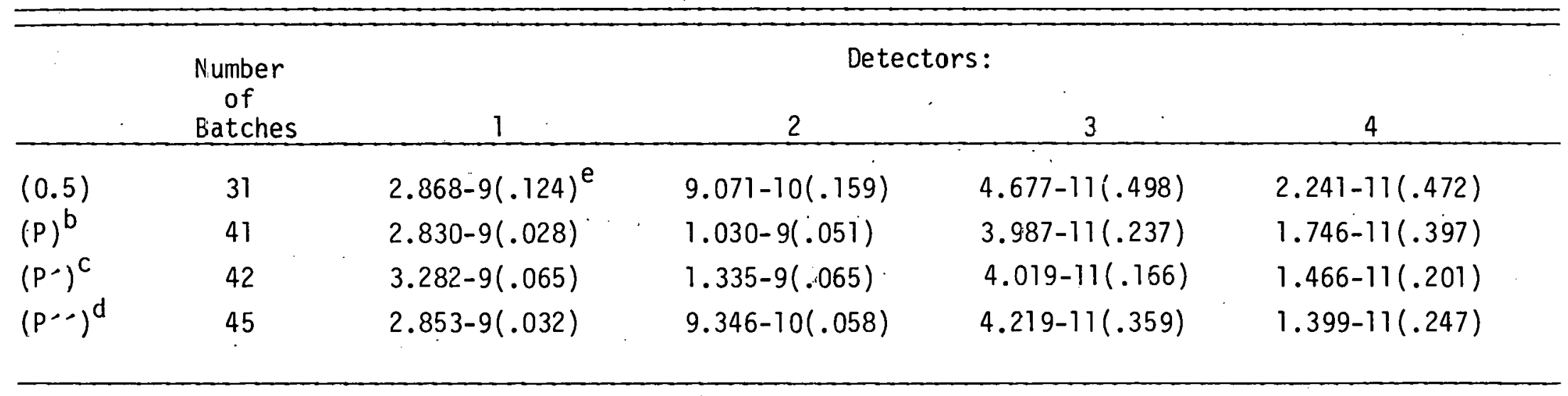

$a_{\text {Unit }}=$ neutrons $/ \mathrm{cm}^{2} /$ sourse neutron.

bath length biasing by the event value $W$ from DOT adjoint calculation with axial adjoint source.

${ }^{C}$ Path length biasing by the point value $x$ * from DOT adjoint calculation with axial adjoint source.

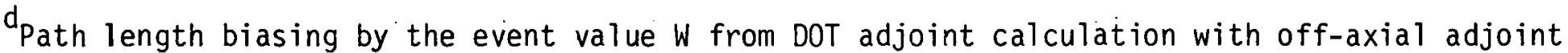
source.

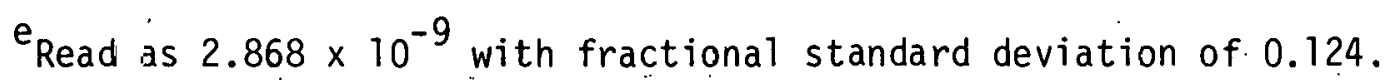


TABLE 7

COMPARISON OF TOTAL NEUTRON FLUX ${ }^{\mathrm{a}}$ OF MONTE CARLO CALCULATIONS USING DIFFERENT POINT VALUES FOR ANGULAR PROBABILITY BIASING

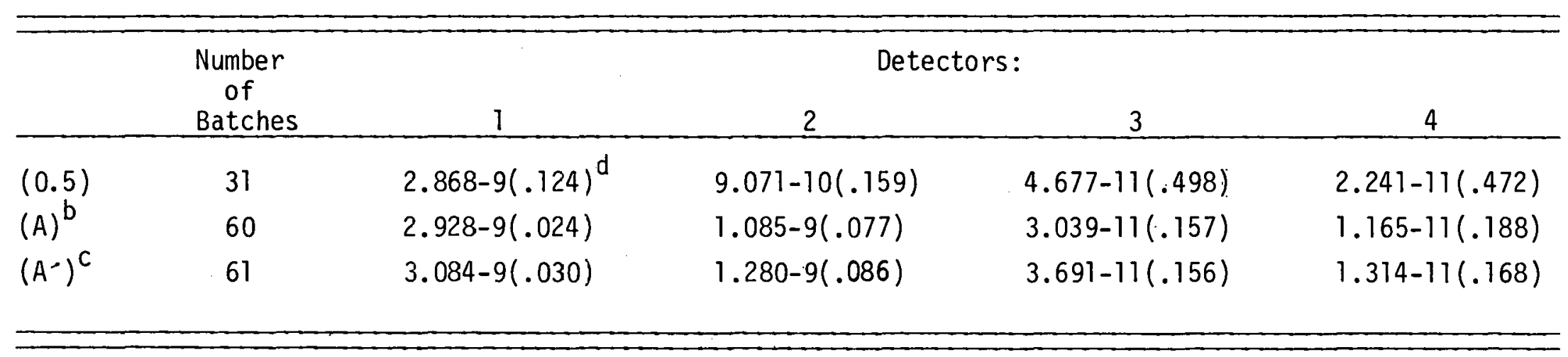

$a_{\text {Unit }}=$ neutrons $/ \mathrm{cm}^{2} /$ source neutron.

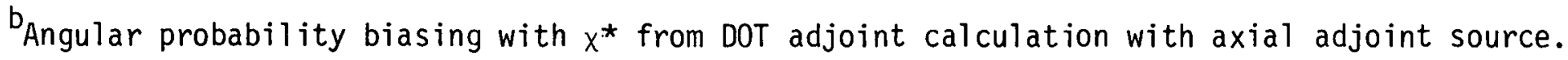

${ }^{C}$ Angular probability biasing with $x^{*}$ from DOT adjoint calculation with off-axial adjoint source.

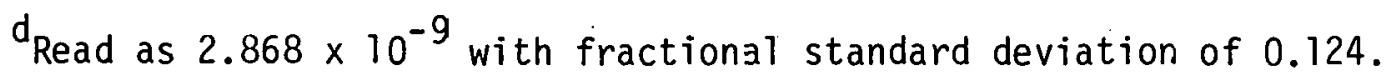


source seems to be equally effective as that obtained from the adjoint DOT calculation with the axial adjoint source. Finally, comparison with $(0.5)$ calculations clearly demonstrates the effectiveness of the angular probability biasing technique. 
CHAPTER VII

\section{CONCLUSIONS AND RECOMMENDATIONS}

Methods of biasing three-dimensional deep penetration Monte Carlo calculations using importance functions obtained from a two-dimensional discrete ordinates adjoint calculation have been developed and systematically evaluated. The important distinction was made between the application of the point value and the application of the event value to bias the transport and the collision processes in Monte Carlo analysis. The biasing techniques developed in this work were the angular probability biasing $(A)$ which altered the collision kernel using the poing value as the importance function and the path length biasing $(P)$ which altered the transport kernel using the event value as the importance function. Source location biasing using the step function (SS) and using the group one scalar adjoint flux obtained from the two-dimensional discrete ordinates adjoint calculation (S) were also investigated.

The angular probability biasing, path length biasing, and source location biasing were applied individually and in various combinations to solve the standard problem. The standard problem consisted of a thick (deep penetration) concrete cylinder with an axial duct (geometric complexity) and a 14-MeV neutron source uniformly distributed over the bottom surface.

Results of calculations using the biasing techniques $(A),(P)$, and (S) were compared with a discrete ordinates solution and two standard Monte Carlo calculations. The calculations using the biasing techniques were shown to be clearly superior to the standard Monte Carlo calculations. 
Based on the FSD's of answers of detectors 1 and 2, the biased calculations were a factor of 2 to 8 better than the (0.5) calculation, and a factor of $1: 5$ to 3.4 better than the calculation with the step source biasing. These improvements clearly demonstrate the applicability and effectiveness of the importance sampling techniques in deep penetration, three-dimensional Monte Carlo calculations.

The comparison of results of Monte Carlo calculations with path length biasing using respectively the event value and the point value as the importance function showed that the event value calculation gave much better statistics (the FSD reduced by a factor of 2) than the point value. Hence, it was concluded that the event value is the appropriate function for altering the transport kernel. The effects of the importance functions, which were obtained from the discrete ordinates adjoint calculation with the off-axial adjoint source, to the biasing techniques were also investigated. Probably due to the nature of the standard problem, these importance functions appeared to be as effective as those obtained from the adjoint calculation with the axial adjoint source.

The techniques of biasing the transport and collision kernels developed in this study were shown to be effective and applicable to the Monte Carlo analysis of three-dimensional deep penetration shielding problems. However, for further research, the following areas are recommended.

1. The effect of higher-urder Legendre expansion of the differential scattering cross section to the angular probability biasing should be studied. Since a 
higher-order expansion allows more scattering directions in the collision process, a more complete utilization of the point value importance information to bias the collision kernel would be possible.

2. Biasing of the azimuthal dependence of the scattering directions should also be studied. One possible approach would be to discretize the cones formed by the scattering polar angles and then bias these discrete directions by the point value.

3. Increase the number of spatial regions of the problem so that better representation and utilization of the two-dimensional adjoint flux could be achieved. This should be particularly helpful to the path length biasing when applied to a problem which has a complex geometry such as the streaming duct.

4. Finally, the possibility of applying these methods to solve more complicated streaming duct problems should be investigated. 


\section{BIBLIOGRAPHY}




\section{BIBLIOGRAPHY}

1. Kahn, Herman, "Application of Monte Carlo," USAEC Report AECU-3259 (Apri1 1954).

2. Goertzel, G., and M. H. Kalos, "Monte Carlo Methods in Transport Problems," Progress in Nuclear Energy, Series I, Physics and Mathematics, II: 315-369 (1953).

3. Coveyou, R. R., V. R. Cain, and K. J. Yost, "Adjoint and Importance in Monte Carlo Application," Nuclear Science and Engineering 27: 219-234 (1967).

4. Kalos, M. H., "Importance Sampling in Monte Carlo Shielding Calculations," Nuclear Science and Engineering 16: 227-234 (1963).

5. Armstrong, T. W., and P. N. Stevens, "A V Importance Function for the Monte Carlo Calculation of Deep Penetration of Gamma Rays," Journal of Nuclear Energy 23: 331-359 (1969).

6. Benda11, D. E., and A. K. McCracken, "McBend - a Prototype Code Utilizing Both Removal-Diffusion and Monte Carlo Methods," Proceedings of the International Conference on the Physics Problems of Reactor Shielding, September 26-29, 1967.

7. Cain, V. R., "Application of $S_{n}$ Adjoint Flux Calculations to Monte Carlo Biasing," Transactions of the American Nuclear Society 10: 399 (1967).

8. Schmidt, F. A. R., E. A. Straker, and V. R. Cain, "Applications of Adjoinl Flux Calculations to Monte Carlo Biasing," Oak Ridge Nationa1 Laboratory Report ORNL-TM-2454 (December 1968).

9. Burgart, C. E., and P. N. Stevens, "A General Method of Importance Sampling the Angle of Scattering in Monte Carlo Calculations," Oak Ridge National Laboratory Report ORNL-TM-2890 (March 1970).

10. Straker, E. A., P. N. Stevens, D. C. Irving, and V. C. Cain, "The MORSE Code-A Multigroup Neutron and Gamma-Ray Monte Carlo Transport Code," Oak Ridge National Laboratory Report ORNL-4585 (September 1970).

11. Roades, W. A., and F. R. Mynatt, "The DOT III Two-Dimensional Discrete Ordinates Transport Code," Oak Ridge National Laboratory Report ORNL-TM-4280 (1973).

12. Irving, D. C., "The Adjoint Boltzmann Equation and Its Simulation by Monte Carlo," Oak Ridge National Laboratory Report ORNL-TM-2879 (May 1970). 
13. Bell, G. I. and S. Glasstone, Nuclear Reactor Theory, Van Nostrand Reinhold Company, New York (1970).

14. Cashwe11, E. D. and C. J. Everett, A Practical Manual on the Monte Carlo Method for Random Walk Problems, Pergamon Press, Inc. New York (1959).

15. Abbott, L. S., "Shielding Against Initial Radiation from Nuclear Weapons," Oak Ridge National Laboratory Report ORNL-RSIC-36 (July 1973).

16. Pace, J. V., III, Union Carbide Corporation - Nuclear Division, Oak Ridge, Tennessee, private communication (1975).

17. Childs, R. L., "GRTUNCL - A Computer Program to Calculate the FirstCollision Source," Union Carbide Corporation, Nuclear Division Report (to be published).

18. Arfken, G., Mathematical Methods for Physists, second ed., Academic Press, Inc., New York (1970).

19. Engle, W..W., Jr., "A User's Manual for ANISN, A One-Dimensional Discrete Ordinates Transport Code with Anisotropic Scattering," Union Carbide Corporation, Nuclear Division Report K-1693 (1967).

20. Lathrop, "DTF-IV, A Fortran-IV Program for Solving the Multigroup Transport Equation with Anisotropic Scattering," Los Alamos Scientific Laboratory Report LA-3373 (1965).

21. Solomito, Milo, Jr., "Methods of Biasing Secondary Gamma-Ray Production in Coupled Neutron Gamma-Ray Monte Carlo Calculations," Ph.D. Dissertation, The University of Tennessee (August 1971).

22. Mynatt, F. R., F. J. Muckenthaler, and P. N. Stevens, "Development of Two-Dimensional Discrete Ordinates Transport Theory for Radiation Shielding," Union Carbide Corporation. Nuclear Division Report CTC-INF-952 (August 1969).

23. Childs, R. L. and V. C. Baker, "FALSTF-A Computer Program to Calculate Activities for Detectors Located External to a Shield," Union Carbide Corporation, Nuclear Division Report (to be published).

24. Spiege1, M. R., Vector Analysis, Schaum Publishing Company, New York (1959).

25. Engle, W. W., Jr., Oak Ridge National Laboratory, Oak Ridge, Tennessee, private communication (1974). 
THIS PAGE

WAS INTENTIONALLY LEFT BLANK 


\section{APPENDIXES}

APPENDIX A

\section{COMPUTER PROGRAMS}

\section{A.1 Input Instruction and Listing of the POINT Program}

The POINT program consists of a main routine and a subroutine SATURN. The input to this program is written in the free-form of

the FIDO format. (19) Presented below is the input instruction and the listing of the program.

1\$\$ array: NAFT, NABC, IREG, JREG, MMDN, NEWDN, NEWMM, NREG T INAFT - logical unit number of the adjoint angular flux tape NABC - logical unit number of the point value tape IREG - number of regions in radial direction JREG - number of regions in axial direction MMDN - number of downward directions in the angular quadrature set NEWDN - number of downward directions with nonzero weights in the angular quadrature set

NEWMM - number of total directions with nonzero weights in the angular quadrature set

NREG - total number of regions, IREG $\times$ JREG

$2 \$ \$$ array: IRLO $(L), L=1$, IREG - mesh number of the lower boundaries of region $L$ in radial direction

$3 \$ \$$ array: $\operatorname{IRUP}(L), L=1$, IREG - mesh number of the upper boundaries of region $L$ in radial direction

$4 \$ \$$ array: IZLO $(L), L=1$, JREG - mesh number of the lower boundaries of region $L$ in axial direction 
$5 \$ \$$ array: $\operatorname{IZUP}(L), L=1$, JREG - mesh number of the upper boundaries of region $L$ in axial direction $T$

C THE POINT PROGRAM

COMMON /BULKBU/ A(1),LI,L2,L3,L4,L5,L6,L7,L8,L9,LL10,L11,LL12,L13

$1 \quad$ L $14, L 15 \cdot L 16, L 17$

2 ,TDOT (18),MM,IM,JM, IG,NIN,NOU

3 , NAFT, NABC, IREG, JREG, MMDN, NEWDN, NEWMM, NREG

4 . DUMMY $(70000)$

DIMENSION LA (1)

EQUIVALENCE (A1),LA(1))

LENGTH $=70050$

DO $5 \mathrm{I}=1$ - LENGTH

$5 \quad A(I)=0$.

NIN $=5$

NOU $=6$

$L 1=43$

$L 2=L 1+8$

NERR $=0$

CALL FIDO(2, IERR, NIN, NOU)

$C * *$ \#DIT PARAMETERS

$L 1 P B=L 1+7$

WRITE (NOU, 1005) (LA II), I =L, I LIPB)

1005 FORMAT 1 OPARAMETERS INPUT FROM CARD .....'

$1 /$ NAFT $=1, I 3,3 x_{,}, L O G$ UNIT \# FOR INPUT ANGULAR FLUX TAPE,

2, 'NABC $=1,13,3 x$, , LOG UNIT \# FOR OUTPUT ANGULAR BIASING FUNCT'

$3 /$ IREG $=1,13,3 \times$, N NUMBER OF REGIONS IN RADIAL DIRFCTION'

41 JREG $=1.13 .3 \times$, INUMBER OF REGIONS IN AXIAL DIRECTION.

$5 \%$. MMDN $=1,13,3 \times$, NUMBER OF DOWNWARD ANGLES IN QUADRATURE SET,

$6 /$ NEWDN $=1,13,3 X, 1$ NEW NUMBER OF DOWNWARD ANGLES '

7, NEWMM $=1,13,3 \times$, NEW NUMBER OF TOTAL ANGLES'

8 , NREG $=1, I 3,3 X$, , TOTAL \# OF REGION, IREG JREG')

C* $\$$ READ THE FIRST RECORD OF ANGULAR FLUX TAPE NAF.T, AND PUT THE IST

C* 18 WARDS INTO TDOT ARRAY, THEN NEST 27 WARDS INTO A(L2) ARRAY

CALL REWNO (NAFT)

CALL WANDR2(NAFT, TDOT, $18, A(L 2), 27,2)$

WRTTE. (NOU, 1010$)$ TUOT

1010 FORMAT ( OODOT DATA TO BE PROCESSED FROM-_...1, 1844)

$M M=L A(L 2+3)$

$I M=L A(L 2+6)$

$J M=L A(L 2+7)$

$I G=L A(L 2+26)$

WRITE (NOU, 1015) MM, IM, JM, IG

1015 FORMAT (POPARAMETERS INPUT FROM DOT-.-.

$1 /$ MM $=1,13,3 \times$, NUMBER OF DIRECTIONS

2, IM $=\because I 3,3 X$, NUMBER OF RADIAL INTERVALS

$3 /$. JM $=1,13,3 \times$, NNUMBER OF $\triangle X$ IAL INTERVALS

4, . IG $=1.13 .3 \times$. 'NUMBER OF ENERGY GROUPS')

CALL CLEARX (A $(L 2), 1,27)$

$L 3=L 2+I R E G$

$L 4=L 3+$ IREG

$L 5=L 4+J R E G$

$L 6=L 5+J R E G$

$L 7=L 6+M M$

$L \theta=L 7+M M$

$L 9=L 8+M M$

$L 10=L 9+M M$

L1 $1=L 10+I M+1$

$L 12=L 11+J M+1$

$L 13=L 12+I R E G$

L $14=L 13+$ JREG

L15=L14+IM MMON

L16=L 15 + IREG * JNHNEWDN

$L 17=L 16+$ IREG $*$ JRE G $\$ N E W M M$ 


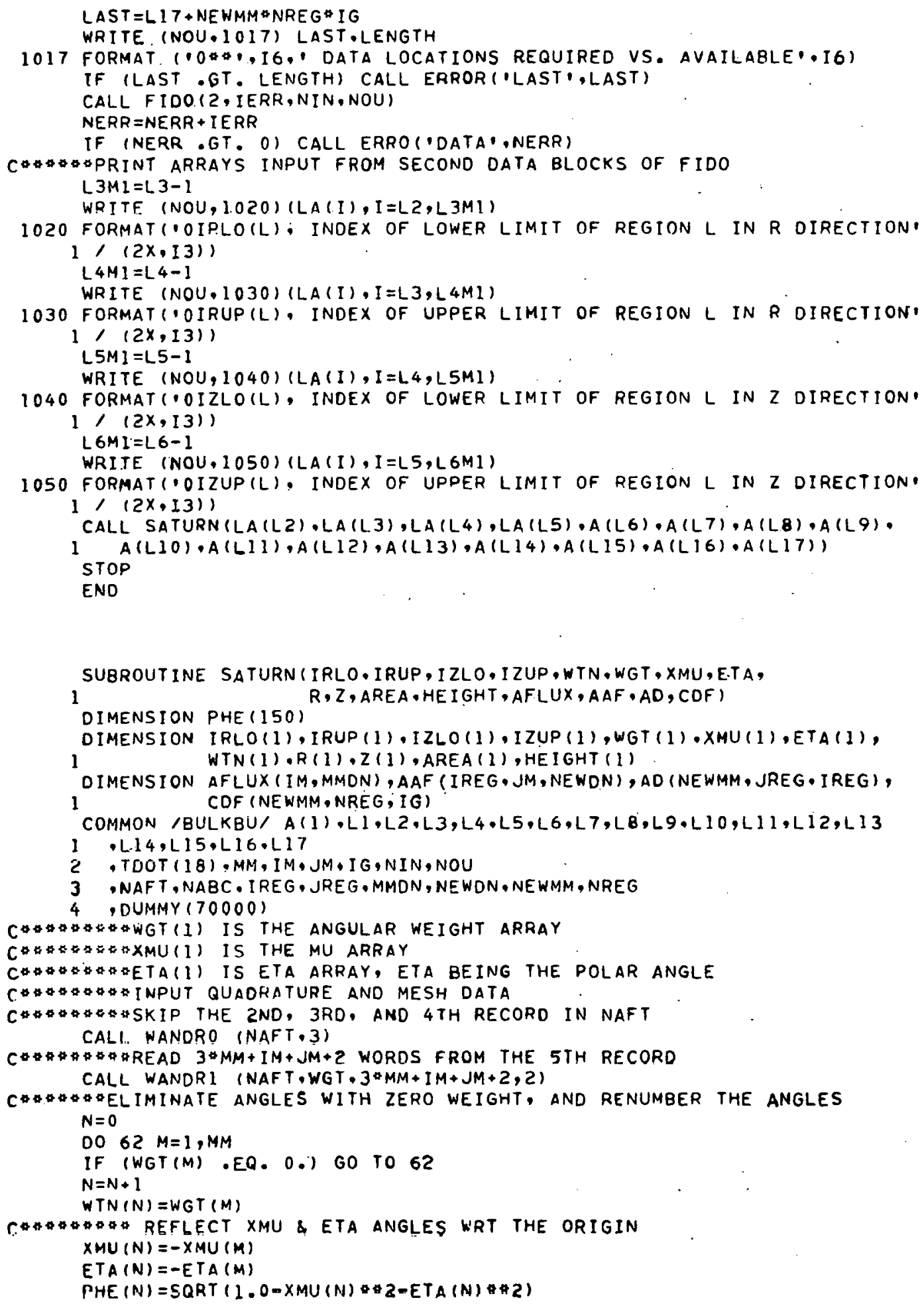




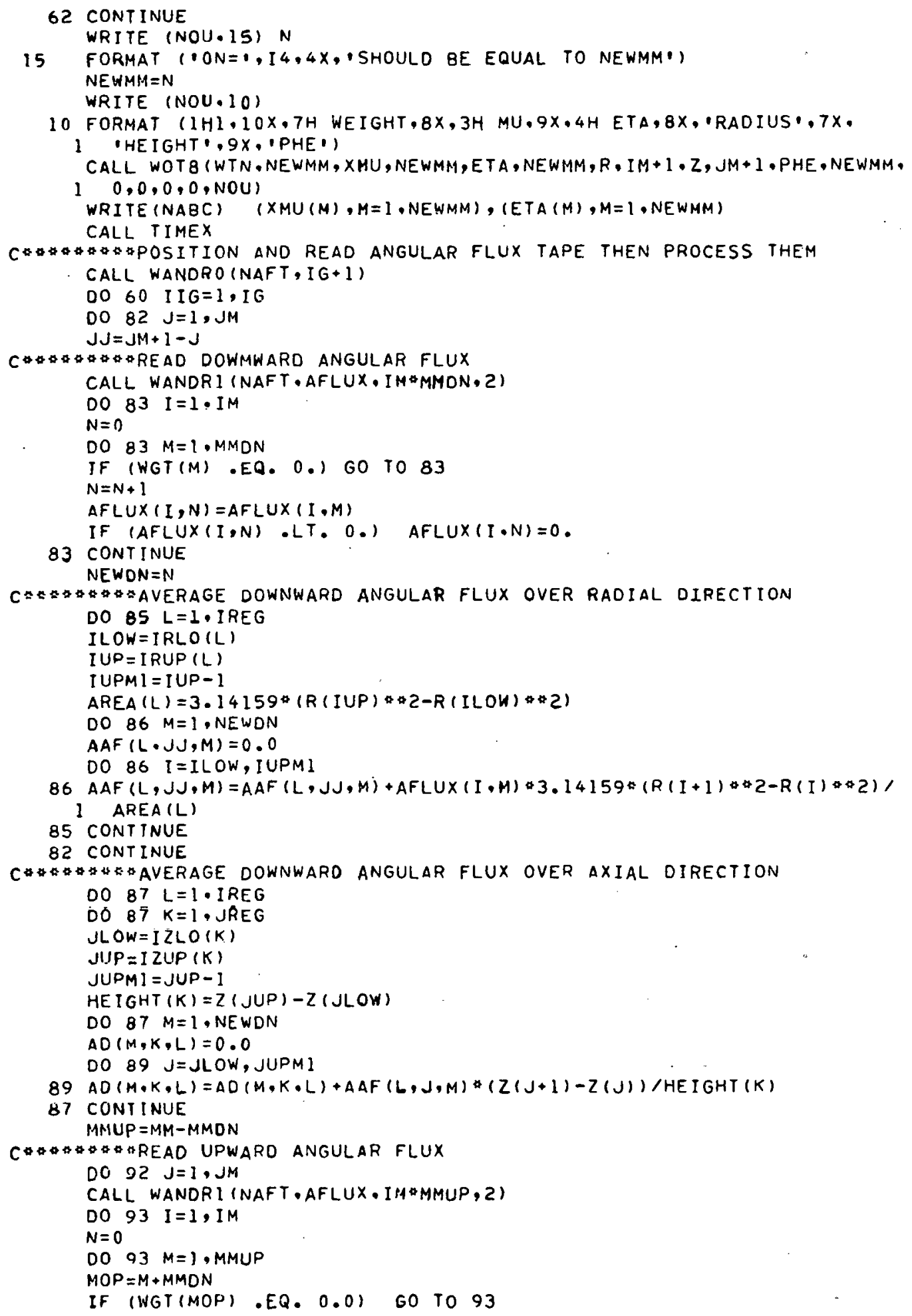




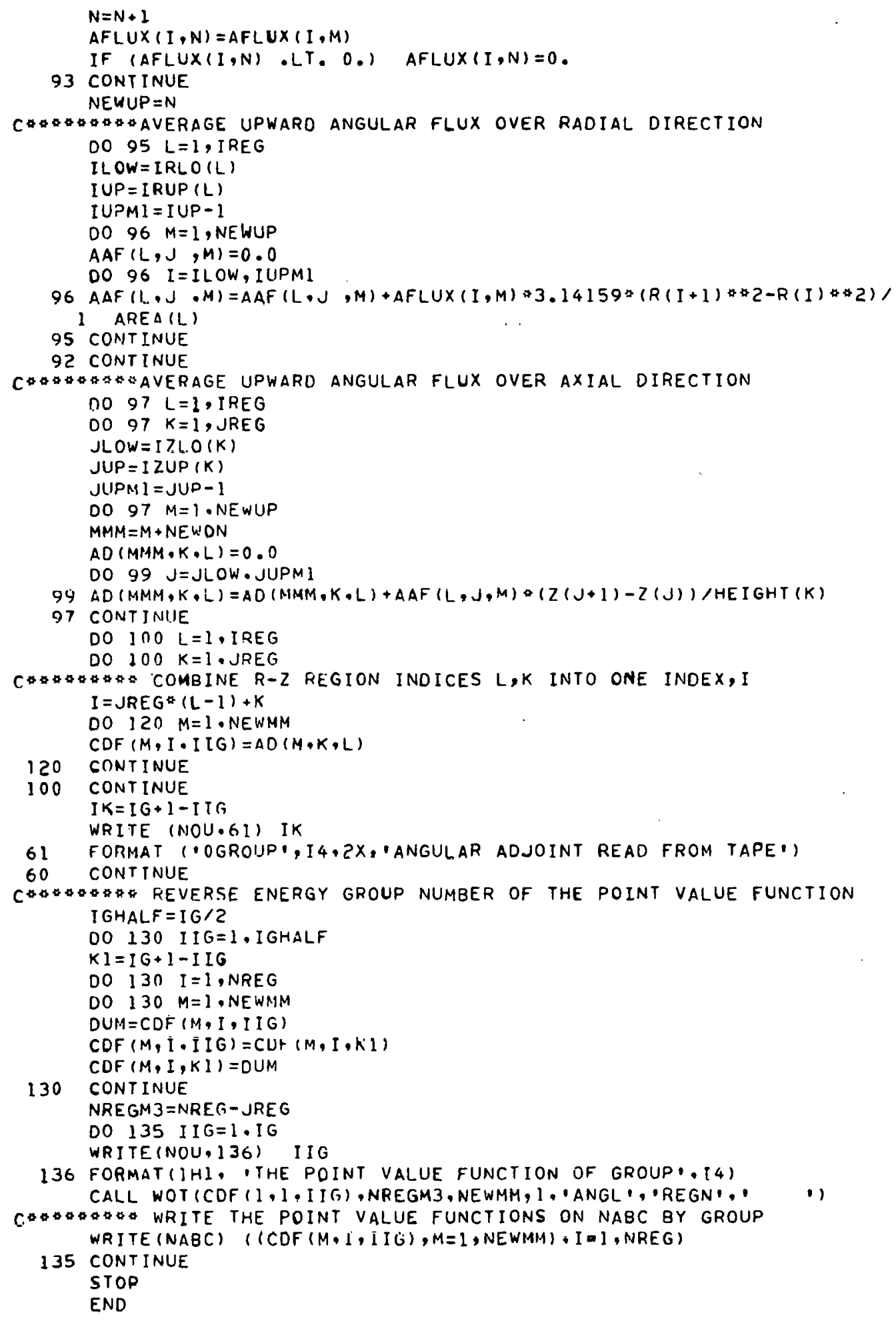




\section{A.2 Input Instruction and Listing of the EVENT Program}

The EVENT program consists of a main routine and subroutines, PEASE and PCON. Like the POINT program, the input to the EVENT program is written in the free-form of the FIDO format. The input instruction and the listing of this program are presented as follows.

1\$\$ array: IGM, IM, JM, MM, NEWMM, IREG, JREG, NREG, LMAX, NIZ4, NEVF T

IGM - number of energy groups

IM - number of radial intervals

JM - number of axial intervals

MM - number of directions in the angular quadrature set NEWMM - number of directions with nonzero weights in the angular quadrature set

IREG - number of regions in radial direction

JREG - number of regions in axial direction

NREG - total number of regions, IREG $\times$ JREG

LMAX - the order of Legendre Polynomial expansion of the differential scattering cross section

NIZ4 - logical unit number for the scattering source tape

INEVF - logical unit number for the event value tape

$2 \star \star$ array: $\operatorname{CST}(I G), I G=1, I G M-$ total macroscopic cross section by

group (input in reversed energy group number)

$3^{* *}$ array: $R(I), I=1, I M+1$ - boundaries of radial intervals

4** array: $Z(J), J=1, J M+1$ - boundaries of axial intervals

5** array: IRLO $(L), L=1$, IREG - mesh number of the lower boundaries of region $L$ in radial direction 
$6^{\star \star}$ array: IRUP $(L), L=1$, IREG - mesh number of the upper boundaries of region $L$ in radial direction

7** array: IZLO $(L), L=1$, JREG - mesh number of the lower boundaries of region $L$ in axial direction

$3^{\star \star}$ array: IZUP $(L), L=1$, JREG - mesh number of the upper boundaries of region $L$ in axial direction

9** array: $W G T(M), M=1, M M$ - weights associated to the directions in the angular quadrature set.

$10^{\star \star}$ array: $X M U(M), M=1, M M$ - direction cosines with respect to the radial direction in the angular quadrature set

11** array: $\operatorname{ETA}(M), M=1$, MM - direction cosines with respect to the axial direction in the angular quadrature set. 
C THE EVENT PROGRAM

COMMON /BULKBU/ A (1) $, L 1, L 2, L 3, L 4, L 5 \cdot L 6, L 7, L 8, L 9, L 10$

,L11,L12,L13,L14,L15,L16,L17,L18,L19,L20

- NIN, NOU I IGM. IM. JM. MM, NEMMM, IREG . JRE G, NREG , LMAX

-NIZL.NEVF

- DUMMY $(64000)$

DIMENSION LA (1)

EQUIVALENCE (A(1),LA(1))

LENGTH $=64000$

$5 \quad A(I)=0.0$

$N I N=5$

NOU $=6$

$L 1=24$

$L 2=L 1+11$

NERR $=0$

C*REAO I\$\$ ARRAY

CALL FIDO (2, IERR, NIN, NOU)

$L I P=L 1+10$

WRITE (NOU, 1005) (LA (I), I =LI, LIP)

1005 FORMATIOPARAMETERS INPUT FROM CARDS.......'

* 1 I IGM $=1$, I $3,3 x$, .NUMBFR OF ENERGY GROUPS

* 1 IM $=1,13.3 x$, NNUMBer of RADIAL INTERVAL.

* 1 jm $=1,13,3 x$, 'Number of axial intervals.

- 1 MM $=1, I 3,3 X$, NUMBER OF DIRECTIONS

- 1 - NEWMM $=1,13,3 x$, NNEW NUMBER OF DIRECTIONS -

* 1 IREG $=1,13,3 x, \cdot *$ OF REgIONS IN RAOIAL DIRECTION

* 1 - JREg $=1,[3.3 x$, *\# OF REgIONS IN axial DIRECTION"

* 1 NREg $=\cdot, I 3,3 x$, 'NUMBer of REgIONS

* ' l lMaX $=1,13,3 x$, imaximum order of scattering.

* 1 NIZ4 = , I3,3x, 'LOg \# FOR SCATTERING SOURCE TAPE.

* 1 ' NEVF $=1,13,3 x$, 'LOg FOR EVENT VALUE TAPE')

ISC $=$ LMAX+I

$L M=(L M A X *(L M A X+3)) / 2$

$L M P 1=L M+1$

$K I M=2 * L M A X+1$

WRITE (NOU.999) ISC,LM,LMPI,KIM

999 FORMAT 1.0 ISC $=1$, I $3,4 X, 1 \quad$ LM $=1, I 3,4 X, 1 \quad$ LMP I $=1,13,4 X$,

1 IKIM $=1, I 3)$

$L 3=L 2+I G M$

$L 4=L 3+I M+I$

$L 5=L 4+J M+1$

$L G=L 5+I R E G$

$L 7=L 6+I K E G$

$L 8=L 7+J R E G$

$L 9=L 8+J R E G$

$L 10=L 9+M M$

$L 11=L 10+M M$

$L 12=L 11+M M$

$L 13=L 12+N E W M M \# M$

$L 14=L 13+L M P 1+N R E G$

LI5 $1514+N E W M M O N R E G$ IGM

LI $16=L 15+N E W M M * I S C *$ ISC

$L 17=L 16+K I M$

$L 18=L 17+N E W M M$

$L 19=L 18+I M \# J M E L M P I$

L20 $=L 19+$ IREG

LAST $=L 20 \rightarrow$ JREG

WRITE (NOU, 1010) LAST, LENGTH

1010 FORMAT $10 \% 1,16,0^{\circ}$ DATA LOCATIONS REQUIRED VS. 
1 AVAILABEL, , I6)

IF (LAST . GT. LENGTH) CALL ERROR('LAST',LAST)

CALL FIDO(2, IERR, NIN, NOU)

$N E R R=N E R R+I E R R$

IF (NERR - GT - O) CALL ERRO('DATA + NERR)

WRITE (NOU 1020$)$

1020 FORMAT (IHI, 10X,' WEIGHTI,8X, ' MU , 9X, ' ETA1.8X,

I IRADIUS , 7X, PHEIGHT ')

CALL WOT8(A (L9), MM,A (LIO), MM,A(LIL),MM,A(L3),IM+I,

$1 A(L 4), J M+1,0,0,0,0,0,0, N D U)$

WRITE (NOU, 1030$)$

1030 FORMAT (IHI,IOX,' CST,.8X,'IRLO',8X,'IRUP', 8X,'IZLO',

$18 X$, 'IZUP')

CALL WOTB(A(L2), IGM, A(L5), IREG, A (L6), IREG, A(L7), JREG,

$1 \quad A(L 8), J R E G, 0,0,0,0,0,0, N O U)$

CALL PEACE (A (L2),A (L3), A (L4), A(L5), A(L6), A(L7), A(LB),

$1 A(L 9), A(L 10), A(L) 11), A(L 12), A(L 13), A(L 14), A(L 15)$,

$2 A(L 16), A(L I 7), A(L 18), A(L 19), A(L 20), L M, L M P I, I S C, K I M)$ STOP

END

SUBROUTINE PEACEICST, R, Z, IRLO, IRUP, IZLO, IZUP, WGT, XMU,

1 ETA,PL, $T, W, P N, B, C P, S S, A R E A, H E I G H T$,

2 (M,LMPI,ISC,KIM)

COMMON /BULKBU/ A(1),LI,L2,L3,L4,L5,L6,L7,L8,L9,LIO

1 ,L11,L12,L13,L14,L15,L16,L17,L18,L19,L20

2 , NIN, NOU, IGM, IM, JM, MM, NEWMM, IREG, JREG, NREG, LMAX

3 , NIZ4,NEVF

DIMENSION CST (1), R(1),Z(1), IRLO(1), IRUP(1), IZLO(1),

1 IZUP(I),WGT (1),XMU(1),ETA(1)

DIMENSION PL (NEWMM,LM), T (LMPI,NREG) ,W (NEWMM.NREG I IGM),

$1 \quad P N(N E W M M$. ISC, ISC), B (KIM), CP (NEWMM), SS (IM, JM, LMPI)

DIMENSION AREA (IREG), HEIGHT (JREG)

CALL PCON (XMU,ETA,WGT,PL,PN, A, CP, NEWMM, I SC,LMAX)

WRITE (NOU, 1050$)$

1050 FORMAT (IHI, ' TESTING PCON $)$

CALL WOT (PL .LM,NEWMM,I, 'ANOL.,PLMC..' $)$

C ELIMINATE ZERO WEIGHT DIRECTIONS. \& REORDER THE DIRECTIONS

C THEN REFLECT XMU \& ETA WITH RESPECT TO THE ORIGIN.

$\mathrm{N}=0$

DO $10 \quad M=1 \cdot M M$

IF (WGT (M) EEQ. 0.1 GO TO In

$N=N+1$

WGT $(N)=W G T(M)$

$X M U(N)=-X M U(M)$

$E T \Delta(N)=-E T \Delta(M)$

10 CONTINUE

WRITE (NOU, $10 \cap 0)$

1000 FORMAT(1H) ' MU \& ETA AFTER REFLECTED')

WFITE (NOU, 1010$)$

1010 FORMAT (1HO.10X,' WEIGHT . 8X, MU , $9 X$, ' ETA')

CALL WOT 8 (WGT, NEWMM, XMU, NEWMM,ET, NEWMM,

$10,0,0,0,0,0,0,0,0,0$, NOU)

CALL PCON(XMU,ETA,WGT,PL,PN, , CP,NEWMM, I SC,LMAX)

WRITE (NOU.1020)

1020 FORMAT (IHI, DISCRETE VALUES OF PLM(THETA) COS(MAPSI) $)$

CALL WOTIPL,LM, NEWMM,I, 'ANGL , IPLMCI, I

$1)$ 


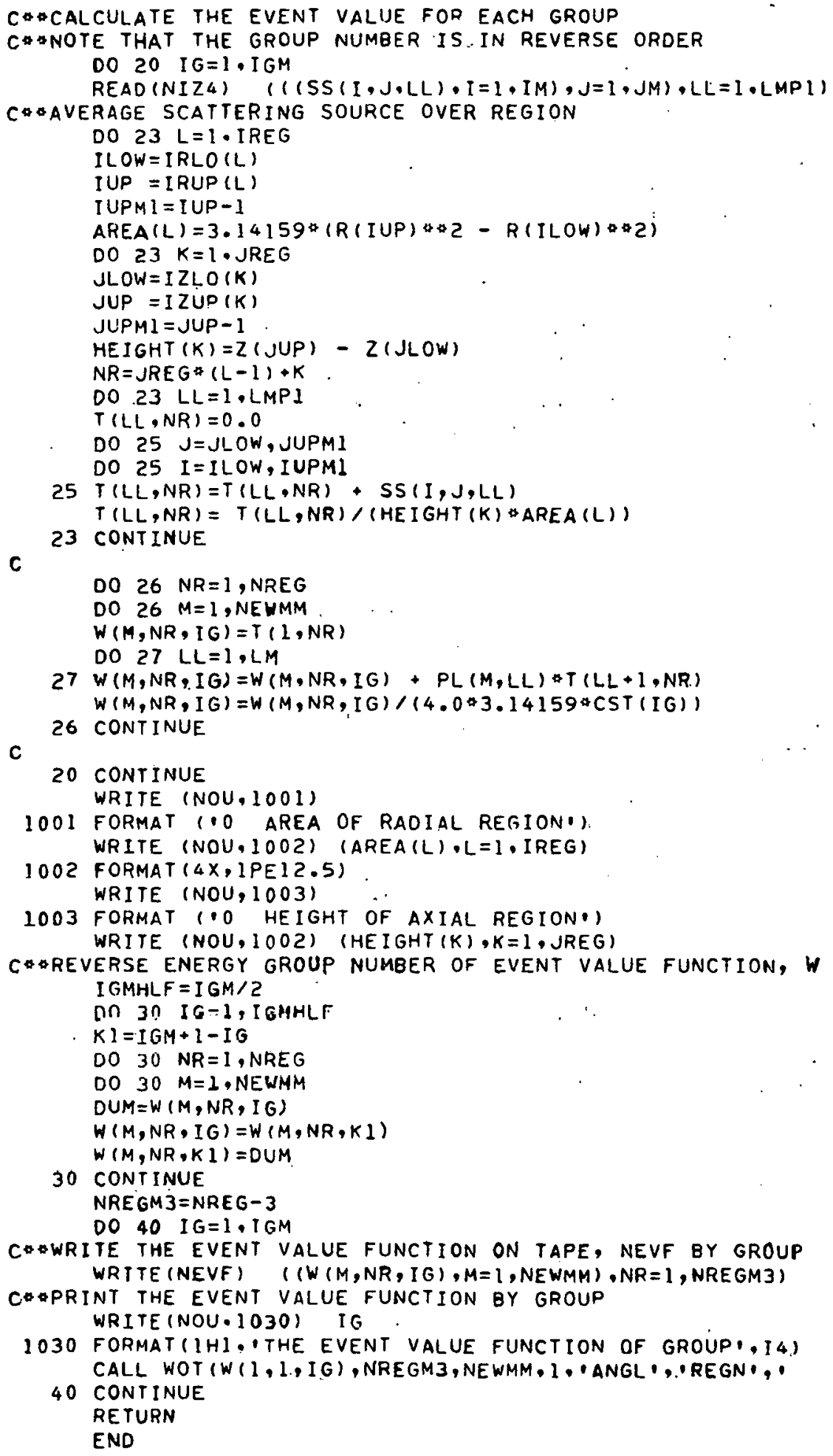




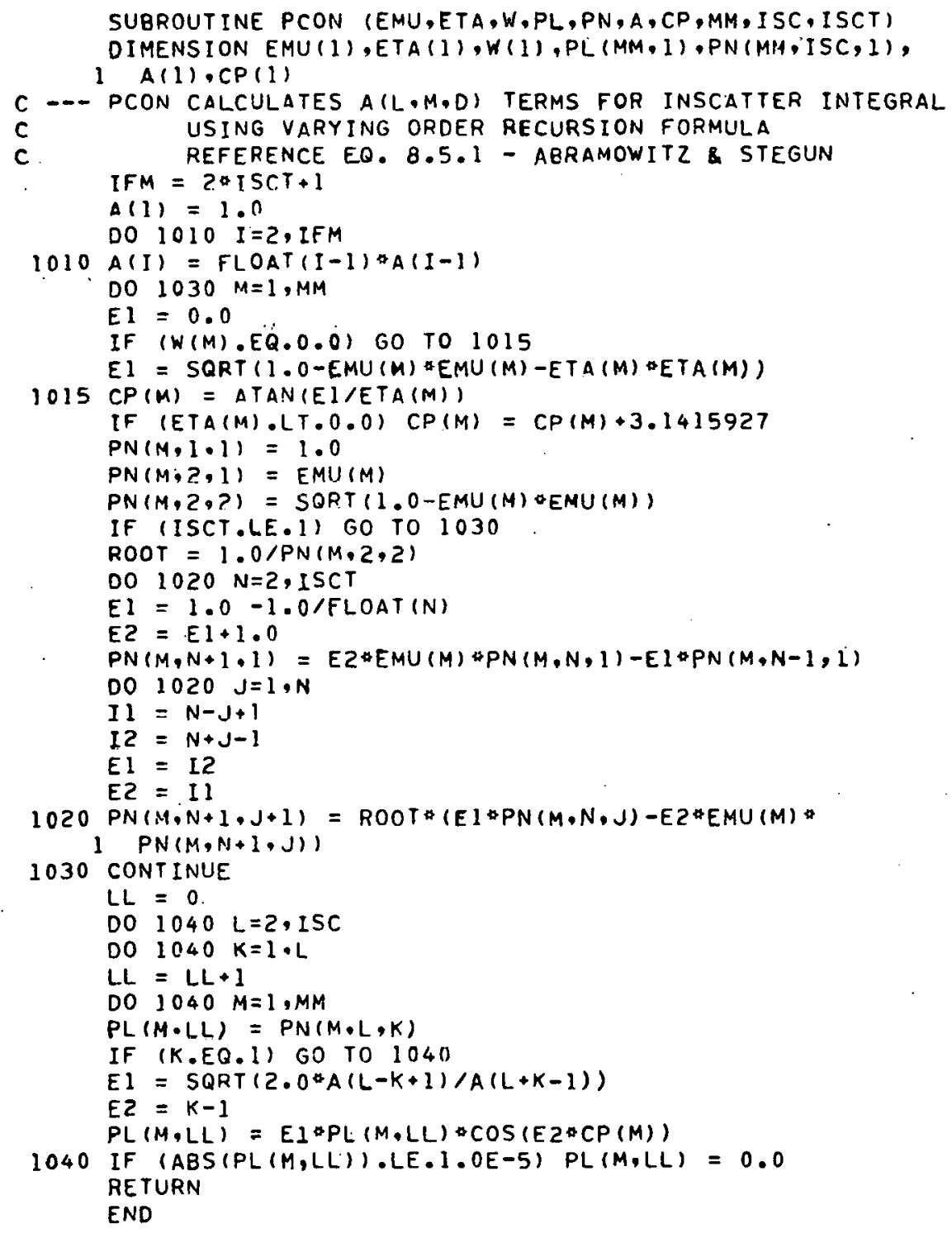




\section{A.3 Subroutines of the Modified MORSE Code}

In the modified MORSE code, the random walk subroutines COLISN and NXTCOL were rewritten in order to incorporate the angular probability biasing and the path length biasing techniques into the MORSE code. Also, the subroutine REGION was introduced to track particle flight trajectories so that path length biasing can be performed. The subroutine INSCOR was written to read the point value tape, the event value tape, or both the point value tape and the event value tape. Finally, two SOURCE subroutines were written. The first one generated. source particle locations according to the source distribution which was biased by the step importance function, and the second one generated source particle locations according to the source distribution which was biased by the DOT adjoint flux.

The listings of these subroutines are presented here in the following order: SOURCE, INSCOR, COLISN, NXTCOL, and REGION. 
SUBROUTINE SOURCE $(I G, U, V, W, X, Y, Z, W A T E, M E D, A G, I S O U R$, 1 ITSTR, NQT3, ODF, ISB, NMTGI

C SOURCE DISTRIBUTION BIASED BY A STEP FUNCTION

$R l=10,0$

RTOL $=150.0$

$C=1000.0$

$X J=(C-1.0) \otimes R l \otimes R l /(R T O L \approx R T O L)+1.0$

$X K=C \otimes R I * R 1 /(R T O L * R T O L \otimes X J)$

RAN $=F L T R N F(0)$

IF (RAN.GT.XK) GO TO 10

RAD $=S Q R T(R A N * X J / C) \approx R T O L$

WATE $=W A T E \backsim X J / C$

GO TO 20

10 RAD $=S Q R T$ (RAN*XJ*RTOL*RTOL- $(C-1.0) * R 1 * R 1)$

WATE $=$ WATE $X J$

20 THETA=2.0*3.14159*FLTRNF(0)

$X=R A O * \operatorname{COS}(T H E T A)$

$Y=R A D * S I N(T H E T A)$

$Z=+0.0001$

C.ALL GIISO $(U, V, W)$

$W=A B S(W)$

RETURN

END

SUBROUTINE SOURCE IIG,U,V,W,X,Y,Z, WATE.MED, AG, ISOUR, 1 I TSTR . NQT 3 D DDF, ISB, NMTG)

C SOURCE DISTRIBUTION BIASED BY DOT ADJOINT FLUX

REAL 4 MI,M2

$M l=0.13155$

$M 2=0.021675$

Pl $=0.954236$

$P 2=0.040994$

RNI = FL TRNF (O)

IF (RN . GT . PI) GO TO 10

C RAOIAL ZONE I IS SELECTED

RN2 =FLTRNF (0)

$R A D=8.0$ *SQRT (RNZ)

WATE $=$ WATE $\$ 0.00298086$

GO TO 111

$10 \quad P I P 2=P 1+P 2$

IF (RNI .GT - PIPZ) GO TO 30

RADIAL ZONE 2 IS SELECTED

RN $3=F L T R N F(0)$

IF (RN3 . GT . 0.48165$) .60 \quad$ TO 25

C

21 ETAI $=$ EXPRNF $(0)$

ETAZ = EXPRNF (O)

$S=(E T A 1+E T A Z) / M 1$

IF(S.GT. 42.0) GO TO 21

GO TO 26

C SELECT FROM EXP(-MS)

25 ETA $=$ AMOD (EXPRNF $(0), 42.0 \otimes M 1)$

$S=E T A / M I$

$26 \quad \mathrm{RAD}=8.0+5$

WATE $=$ WATE $0.2533732 * E \times P(M I * S)$

GO TO 111

C RADIAL ZONE 3 IS SELECTED

$30 \quad$ RN4 $=F L T R N F(0)$ 


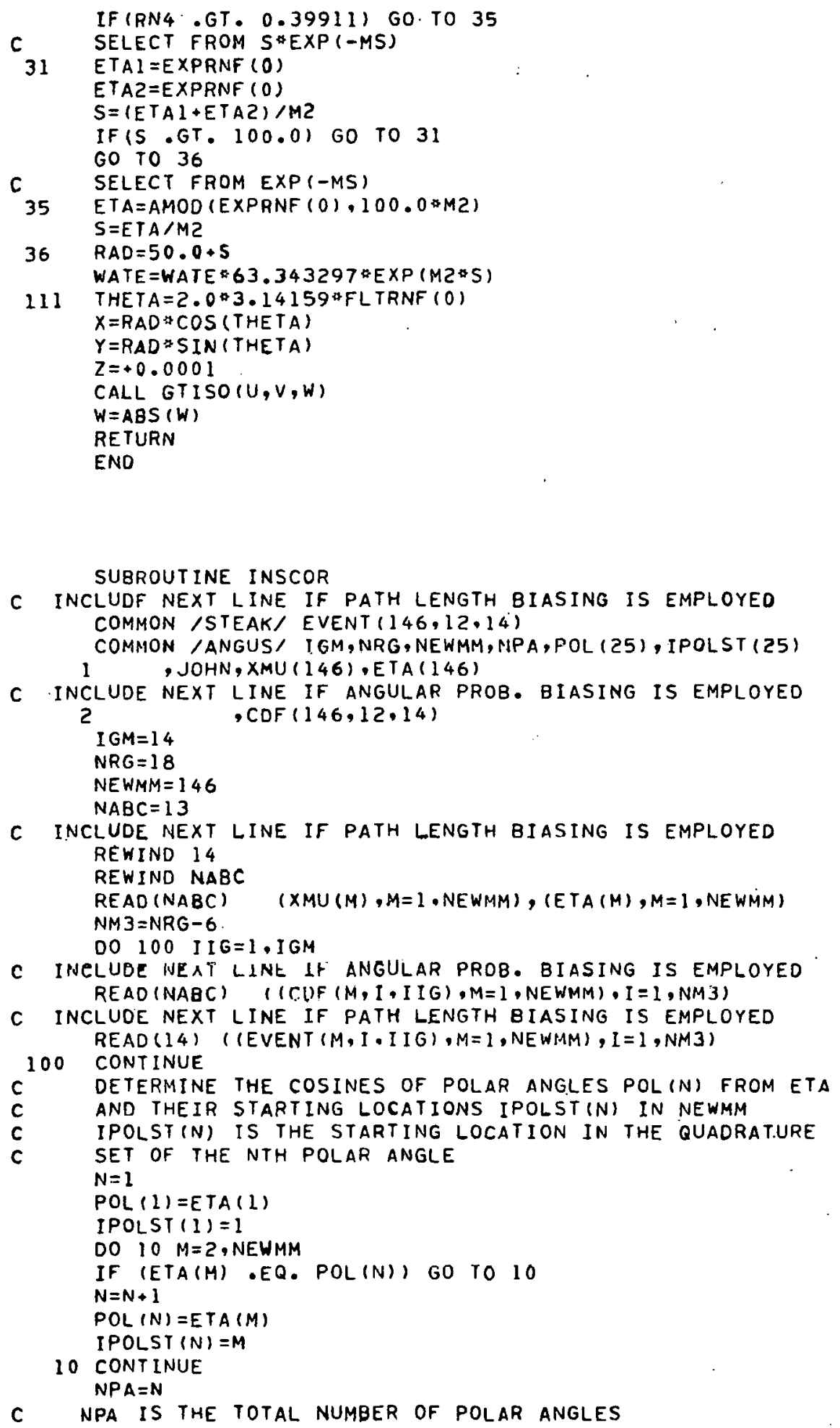


WRITE $(6,105)$

105 FORMAT('OPOLAR ANGLES, POL (N)') WRITE $(6,106)$ (POL (N), N=l,NPA)

106 FORMAT $(2 X, 1$ PBE 14.5$)$ WRITE $(6.107)$

107 FORMAT ( OINDEX OF STARTING POLAR ANGLES, IPOLST(N) WRITE $(6,108)$ (IPOLST $(N), N=1, N P A)$

108 FORMAT $(2 X, 8$ I 14$)$ RETURN END

SUBROUTINE COLISN(IG,U,V,W,WATE, IMED, NREG)

C THIS ROUTINE IS CALLED AT EACH COLLISION

C IT CONVERTS INCOMING GROUP NO., DIRECTION COSINES AND

C PARTICLE WEIGHT INTO POST COLLISION PARAMETERS COMMON /ANGUS/ IGM, NRG, NEWMM, NPA,POL (25), IPOLST (25) 1 , JOHN, XMU (146), ETA (146)

2 , CDF $(146,12,14)$

COMMON /USER/ JUNK(22), NMTG

COMMON /NUTRON/ DUH $(13) \cdot X \cdot Y, Z$ COMMON/LOCSIG/ISTART, ISCCOG, INABOG, IGABOG, IFPORG, 1 IFNGP, IF SPOG, IDSGOG. IPRBNG, IPRBGG, ISCANG, ISCAGG, ISPORG, ISPORT, INPBUF, IS IGOG, INFPOG, I $\triangle B S O G$, ITOTSG, NGP, NDS, NGG, NDSG, INGP, INDS, NMED, NELEM, NMIX, NCOEF, NSCT, NTS, NT G, NDSNGP, NOSNGG, I ADJ, NME, LOC, INGS, INSG, I ], IO,KKK, IXTAPE, I DEL, I TEML, I TEMG, IRSG, IROSG, ISTR, IPRIN, IFMU, IMOM, IOTF, ISTAT, IPUN, NUS, NGN, THT, INUS. INUSN, INGN, INGNP, INNN, I GGG

COMMON SIGT(I)

DIMENSION SICK (5), POF (5), A (5), B(5), C (5), KNOX (5)

DIMENSION NSIG $(1)$

EQUIVALENCE (SIGT(1), NSIG(1))

CALL GTMED (IMEO, MED)

IGMED $=(M E D-1)$ *ISPORG + IG

PNAB = SIGT (IGMED + INABOG)

WATE = WATE PNAB

IF (IG-NGP) $10,10,15$

$10 \quad I H=I G$

NOSK $=N S I G(I N N N+I G)$

IS $=(M E D-1) * I S P O R G+I F S P O G+N S I G(I N G S+I G)$

NADDPG $=0$

ITE $=$ NSIG (INGS+IH)

GO TO 20

15 NADDPG =IPRBGG-IPRBNG

$I H=I G-N G P$

NDSK =NSIG(IGGG+IH)

I TE =NS I G (INSG I IH)

IS $=(M E D-1) \cdot I S P O R G+I D S G O G+I T E$

20 IF $L O C$

$125,25,35$

$C 9=0$

$R=F L T R N F(0)$

DO $30 \quad I=1$-NDSK

$C 9=C 9+\operatorname{SIGT}(I S+I)$

IF (C9-R) $30,40,40$

30 CONTINUE

$I=N O S K$

60 TO 40

35 IND $=$ LOC

$+N T G{ }^{2}(N R E G-1)$ 


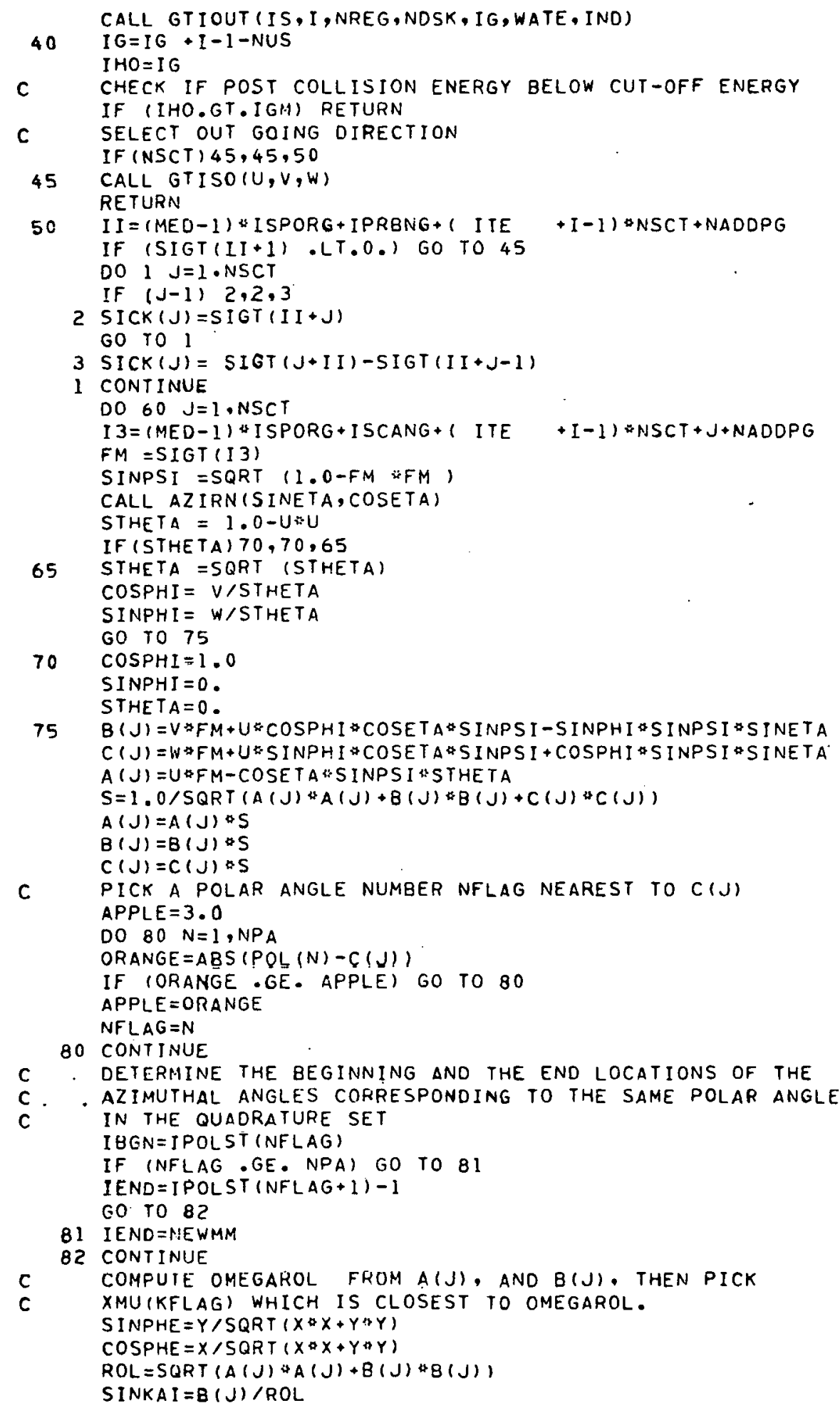




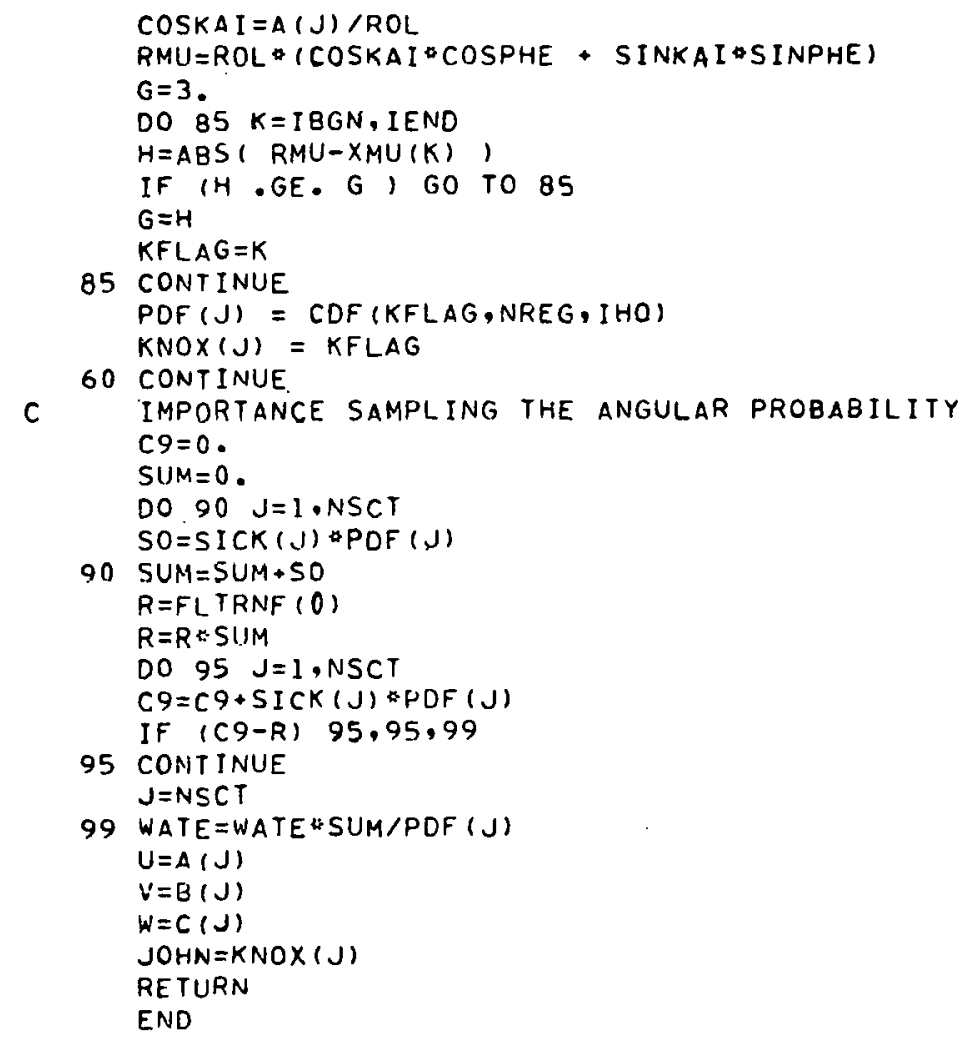

SUBROUTINE NXTCOL

C THIS ROUTINE IS FOR BIASING OF NEXT COLLISION SITE BY

C EVENT VALUE FROM DOT CALCULATION

COMMON /STEAK/ EVENT $(146,12,14)$

COMMON /ANGUS/ IGM,NRG, NEWMM, NPA,POL (25), IPOLST (25)

1 , JOHN, XMU (146)

COMMON /RIBEYE/ NEWREG(20), ARG (20), XX(20), YY (20),

1

$Z Z(20)$, CSET $(20)$, IZONE $(20), M E O(20)$

COMMON /APOLLO/ AGSTRT, DOF, DEADWT (5), ETA,ETATH,ETAUSD, 1 UNIP, VINP, WNIP, WTSTRT, XSTRT, YSTRT, ZSTRT, TCUT, XTRA $(10)$, 2 IO, I I, MEDIA, I AD JM, ISBIAS, ISOUR, I TERS, ITIME , ITSTR,

3LOCWIS, LUCFWL. LOCEPR, LOCNSC . LOCF SN, MAXSP. MAXT TM, MEDALB. 4 MGPREG, MXREG, NALB , NOEAD (5), MEWNM , NGEOM, NGPQT I NGPQT2,

5 NGPQT 3 , NGPOTG, NGPQTN, NI TS, NKCALC, NK ILL, NLAST, NMEM,

6 NMGP, NAOST, NMTG, NOLEAK, NORMF, NPAST, NPSCL (13), NQUIT .

7 NSIGL, NSOUR, NSPLT, NSTRT, NXTRA (10)

COMMON INUTRON/ NAME . NAMEX, IG, IGO, NMED, MEDOLD, NREG .

$1 \mathrm{U}, V, W, U O L D, Y O L D, W O L D, X, Y, Z, X O L D, Y O L D, Z O L D, W A T E, O L D W T$,

2 WTBC, IBLZN, IBLZZO, AGE, OLDAGE

COMMON VEL (1)

DIMENSION PROB $(20), 0(20)$

C* IF ANGULAR PROB. BIASING IS ALSO EMPLOYED, INCLUDE

C* THF FOLLOWING 3 LINES.

OATA NM/O/

IF (NAME.EQ. NM) GO TO 99 
NM $=$ NAME

C** PICK a POLAR ANGLE NUMBER NFLAG NEAREST TO WOLD

$\triangle P P L E=3.0$

DO $80 \mathrm{~N}=1, N P A$

ORANGE = ABS (POL $(N)-W O L D)$

IF (ORANGE . GE. APPLE) GO TO 80

APPLE =ORANGE

NFL $A G=N$

80 CONTINUE

C * DETERMINE THE BEgINNING AND THE END LOCATIONS OF THE

C* AZIMUTHAL ANGLES CORRESPONDING TO THE SAME POLAR

$C *$ aNGLE IN THE QUADRATURE SET

IBGN = IPOLST (NFLAG)

IF (NFLAG. GE. NPA) GO TO 81

IEND = IPOLST $(N F L A G+1)-1$

GO TO 82

81 IEND=NEWMM

82 CONTINUE

C*\$ COMPUTE RMU FROM UOLD, AND VOLD, THEN PICK XMU(KFLAG)

$C *$ WHICH IS CLOSEST TO RMU.

SINPHE $=Y O L D / S Q R T(X O L D * X O L D+Y O L D * Y O L D)$

COSPHE $=X O L D / S Q R T(X O L D \approx X O L D+Y O L D * Y O L D)$

$R O L=S Q R T(U O L O \approx U O L D+V O L D * V O L D)$

SINKAI $=$ VOLD/ROL

COSKAI $=U O L D / R O L$

$R M U=R O L$ (COSKAI COSPHE + SINKAI \$SINPHE)

$G=3$.

DO B5 $K=I B G N$, IEND

$H=A B S$ ( RMU-XMU(K) )

If $(H \cdot G E \cdot G)$ GO TO 85

$G=H$

JOHN $=K$

85 CONTINUE

$C *$ IF ANGULAR PROB. BIASING IS ALSO EMPLOYED, INCLUDE

$C *$ THE FOLLOWING LINE.

99 CONTINUE.

WTBC =WATE

CALL REGION $X O L D, Y O L D, Z O L D, U O L D, V O L D, W O L D$, IGO, MEDOLD,

1 IBLZN, NREG, IRG, IESCAP)

IF (IESCAP.GE. I) GO TO 999

C IESCAP EQUALS TO I WHEN DIFFICULT $\%$ OCCURREO IN IRACKING

C THROUGH THE GEOMETRY IN SUBROUTINE REGION

C THUS TREAT THE PARTICAL AS AN ESCAPE

C IRG IS THE NUMBER OF REGIONS THE TRAJECTORY WILL GO

C THROUGH. BEFORE REACHING THE EXTERNAL VOID

$N R=N E W R E G(1)$

$M=J O H N$

IF (ARG (IRG) + 0.000001$) 55,999,999$

55 PEXT =EXP(ARG (IRG))

$Q(1)=E V E N T(M, N R, I G O) *(1,0-E X P(A R G(1)))$

DO IOO $I=2, I R G$

$N R=N E W R E G$ (I)

$Q(I)=E V E N T(M, N R, I G O) *(\operatorname{EXP}(A R G(I-1))-E X P(A R G(I)))$

100 CONTINUE

QSUM $=0.0$

DO $110 \quad I=1$. IRG

$110 \quad Q S U M=Q S U M+Q(I)$

FNORM $=Q S U M /(1 \cdot 0-P E X T)$

$00120 \quad I=1 \cdot I R G$

120 PROB (I) $=Q(I) / F N O R M$

$P R O B(I R G+1)=P E \times T$ 
$I R G P I=I R G+1$

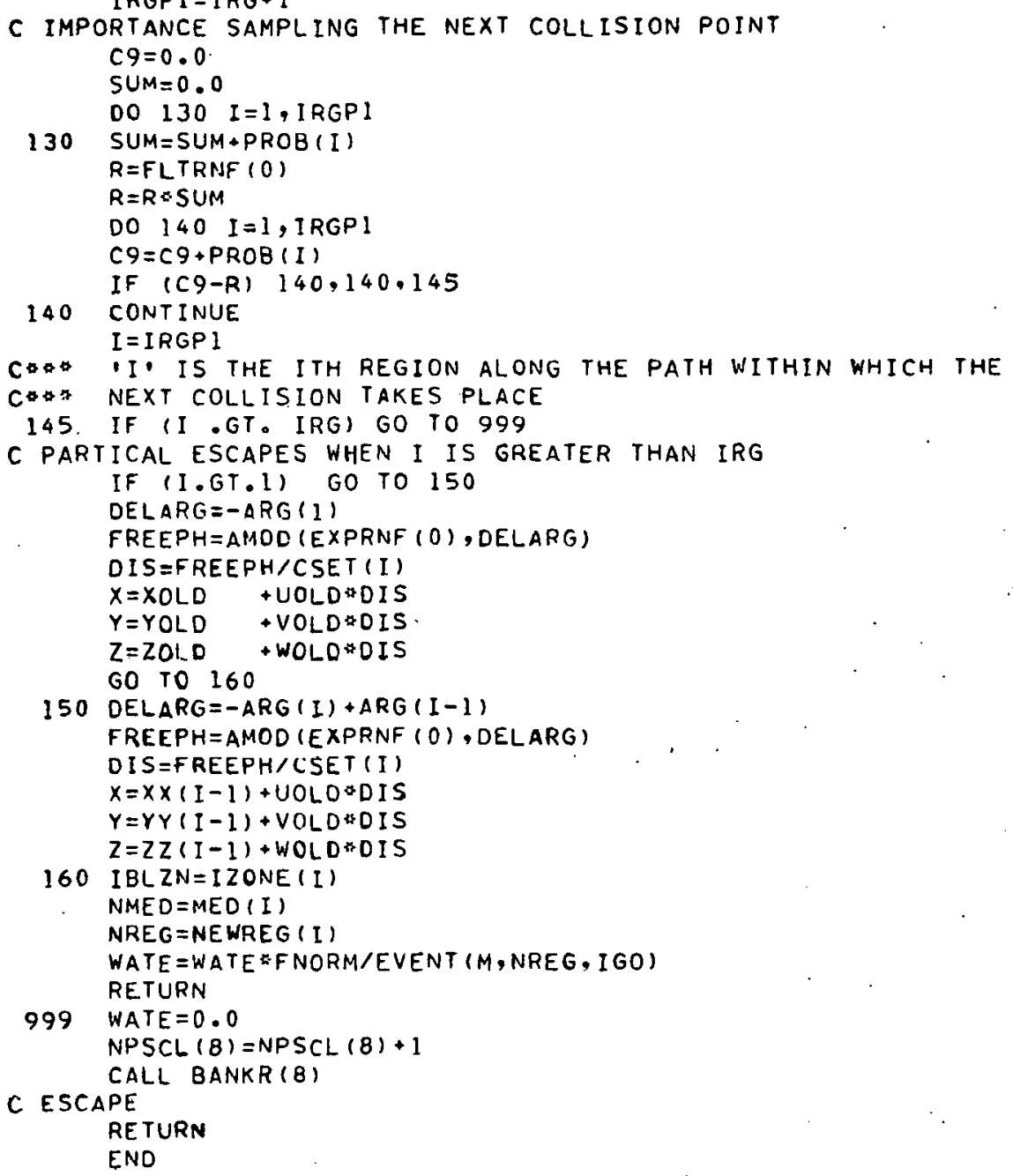

SUBROUTINE REGION $(X I, Y I, Z I, U, V, W, I G, M E D I U M, I B L Z$ NREGN, 1 IRG. IESCAP) 


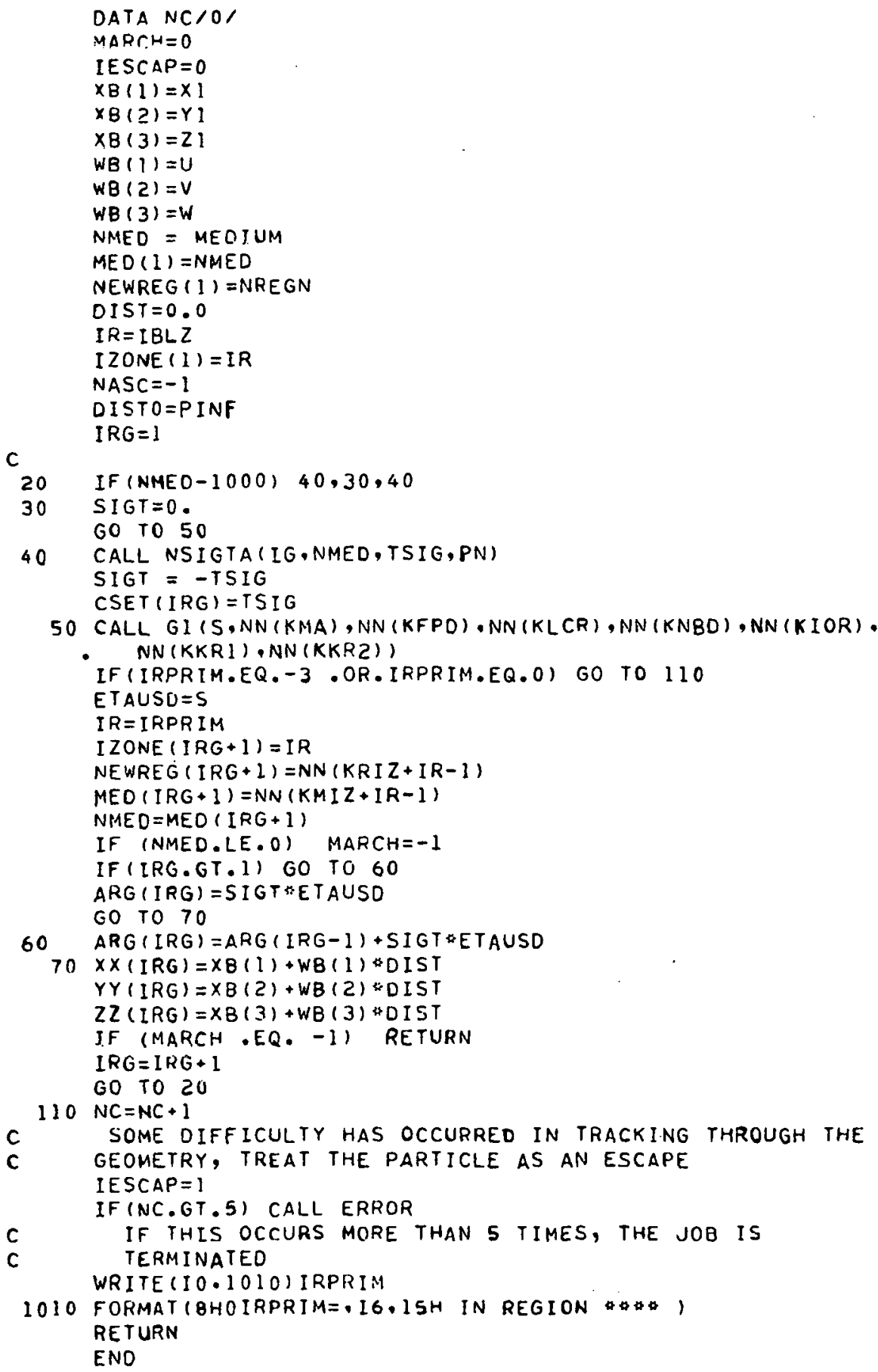

50 CALL GI (S, NN (KMA), NN (KFPO), NN (KLCR), NN (KNBD), NN (KIOR), 


\section{COORDINATE TRANSFORMATIONS}

The purpose of this appendix is to describe how the components of the direction vector $\bar{\Omega}$ are transformed from the cylindrical coordinate system to those of the rectangular coordinate systein, and vice versa.

The direction vector $\bar{\Omega}$ at a point $\bar{P}(x, y, z)$ can be expressed in terms of its components in the cylindrical coordinate system as

$$
\begin{aligned}
\bar{\Omega} & =\bar{\Omega}_{R}+\bar{\Omega}_{\phi}+\bar{\Omega}_{z} \\
& =\Omega_{R} \hat{e}_{R}+\Omega_{\phi} \hat{e}_{\phi}+\Omega_{z} \hat{e}_{z},
\end{aligned}
$$

where $\hat{e}_{R}, \hat{e}_{\phi}$ and $\hat{e}_{z}$ are the unit direction vectors in the cylindrical coordinate system. From Figures 7 , page 36 , and Figure 17, it can be seen that

$$
\begin{aligned}
\Omega_{\mathrm{R}} & =\bar{\Omega} \cdot \hat{\mathrm{e}}_{\mathrm{R}}=\cos \psi \equiv \varepsilon \\
s_{\mathrm{Z}} & =\bar{\Omega} \cdot \hat{\mathrm{e}}_{\mathrm{Z}}=\cos \gamma \equiv \zeta \\
\Omega_{\phi}^{2} & =\Omega_{\rho}^{2}-\Omega_{\mathrm{R}}^{2} \\
\therefore \quad \Omega_{\phi} & = \pm\left(s_{\rho}^{2}-s i_{\mathrm{R}}^{2}\right)^{1 / 2} .
\end{aligned}
$$


ORNL-DWG 75-17719

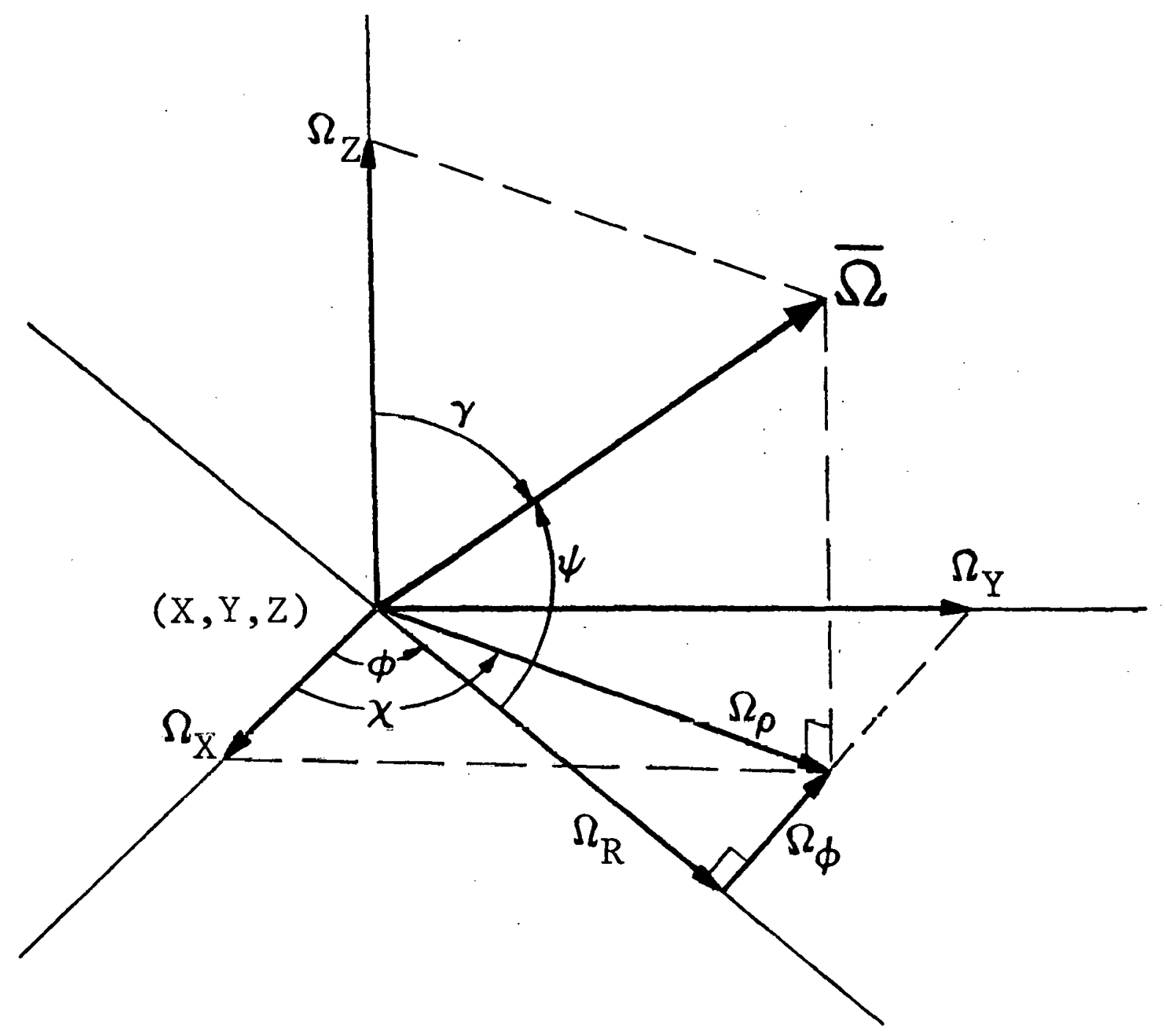

Figure 17. The Direction Vector $\bar{\Omega}$ and Its Components. 
Substituting $\Omega_{\rho}=\sin \gamma$ and $\Omega_{R}=\varepsilon$ into the above equation gives

$$
\begin{aligned}
\Omega_{\phi} & = \pm\left(\sin ^{2} \gamma-\varepsilon^{2}\right)^{1 / 2} \\
& = \pm\left(1-\cos ^{2} \gamma-\varepsilon^{2}\right)^{1 / 2} \\
& = \pm\left(1-\zeta^{2}-\varepsilon^{2}\right)^{1 / 2} .
\end{aligned}
$$

The position vector of the point $\bar{P}$ is

$$
\begin{aligned}
\bar{p} & =x \hat{i}+y \hat{j}+z \hat{k} \\
& =R \cos \phi \hat{i}+R \sin \phi \hat{j}+z \hat{k} .
\end{aligned}
$$

The tangent vectors to the $R, \phi$, and $z$ curves are given respectively by $\frac{\partial \bar{P}}{\partial R}, \frac{\partial \bar{P}}{\partial \phi}$, and $\frac{\partial \bar{P}}{\partial z}$ where

$$
\begin{aligned}
& \frac{\partial \bar{P}}{\partial R}=\cos \phi \hat{i}+\sin \phi \hat{j} \\
& \frac{\partial \bar{P}}{\partial \phi}=-R \sin \phi \hat{i}+R \cos \phi \hat{j} \\
& \frac{\partial \bar{P}}{\partial z}=\hat{k} .
\end{aligned}
$$

The unit vectors in these directions are ${ }^{(24)}$

$$
\begin{aligned}
& \hat{e}_{R}=\frac{\frac{\partial \bar{P}}{\partial R}}{\left|\frac{\partial \bar{P}}{\partial R}\right|}=\frac{\cos \phi \hat{i}+\sin \phi \hat{j}}{\left(\cos _{\phi}{ }^{2}+\sin ^{2} \phi\right)}=\cos \phi \hat{i}+\sin \phi \hat{j} \\
& \hat{e}_{\phi}=\frac{\frac{\partial \bar{P}}{\partial \phi}}{\left|\frac{\partial \bar{P}}{\partial \phi}\right|}=\frac{-R \sin \phi \hat{i}+R \cos \phi \hat{j}}{\left(R^{2} \sin ^{2} \phi+R^{2} \cdot \cos ^{2} \phi\right)^{1 / 2}}=-\sin \phi \hat{i}+\cos \phi \hat{j}
\end{aligned}
$$




$$
\hat{e}_{z}=\hat{k} \text {. }
$$

Substituting Equations (B-2) through (B-7) into Equation (B-1) yields

$$
\begin{aligned}
\bar{\Omega} & =\varepsilon(\cos \phi \hat{i}+\sin \phi \hat{j}) \pm\left(1-\zeta^{2}-\varepsilon^{2}\right)^{1 / 2}(-\sin \phi \hat{i}+\cos \hat{j})+\zeta \hat{k} \\
& =\left(\varepsilon \cos \phi \overline{7} \sqrt{1-\zeta^{2}-\varepsilon^{2}} \sin \phi\right) \hat{i}+\left(\varepsilon \sin \phi \pm \sqrt{1-\zeta^{2}-\varepsilon^{2}} \cos \phi\right) \hat{j}+\zeta \hat{k} .
\end{aligned}
$$

Hence, the components of $\bar{\Omega}$ in the rectangular coordinate system is

$$
\begin{aligned}
\Omega_{x} & =\bar{\Omega} \cdot \hat{i} \\
& =\varepsilon \cos \phi \mp \sqrt{1-\zeta^{2}-\varepsilon^{2}} \sin \phi \\
\Omega_{y} & =\bar{\Omega} \cdot \hat{j} \\
& =\varepsilon \sin \phi \pm \sqrt{1-\zeta^{2}-\varepsilon^{2}} \cos \phi \\
\Omega_{z} & -\bar{\Omega} \cdot \hat{k} \\
& =\zeta
\end{aligned}
$$

where $\sin \phi=\frac{y}{\sqrt{x^{2}+y^{2}}}$ and $\cos \phi=\frac{x}{\sqrt{x^{2}+y^{2}}}$.

Equations $(B-8),(B-9)$, and $(B-10)$ are the relationships for transformation of the direction vector $\bar{\Omega}$ at the point $\bar{P}(x, y, z)$ from the cylindrical corrdinate system to the rectangular coordinate system. Note that for each set of cylindrical components $\zeta$ and $\varepsilon$, these are two corresponding sets of rectangular components $\Omega_{x}, \Omega_{y}$, and $\Omega_{z}$. In a DOT calculation 
with R-Z geometry, $\zeta$ and $\varepsilon$ are respectively the direction cosines with respect to the $Z$-axis and the R-axis.

Given the rectangular components $\Omega_{x}, \Omega_{y}$, and $\Omega_{z}$ of the direction vector $\bar{\Omega}$ at the point $\bar{P}(x, y, z)$. The transformation of $\bar{\Omega}$ from the rectangular coordinate system into the cylindrical components $\zeta$ and $\varepsilon$ in the cylindrical coordinate system is performed as follows. From Equation $(B-10), \zeta$ is simply equal to $\Omega_{z}$. Furthermore, from Figure 17,

$$
\begin{aligned}
\varepsilon \equiv \Omega_{R} & =\Omega_{\rho} \cos (x-\phi) \\
& =\Omega_{\rho}(\cos x \cos \phi+\sin x \sin \phi)
\end{aligned}
$$

where

$$
\cos x=\frac{\Omega_{x}}{\Omega_{\rho}}, \quad \sin x \frac{\Omega_{y}}{\Omega_{\rho}},
$$

and

$$
\cos \phi=\frac{x}{\sqrt{x^{2}+y^{2}}}, \sin \phi=\frac{y}{\sqrt{x^{2}+y^{2}}} .
$$

Substituting the above quantities into Equation (B-11), gives

$$
\begin{aligned}
\varepsilon & =\Omega_{\rho}\left[\frac{\Omega_{0}}{\Omega_{\rho}} \frac{x}{\sqrt{x^{2}+y^{2}}}+\frac{\Omega_{y}}{\Omega_{\rho}} \frac{y}{\sqrt{x^{2}+y^{2}}}\right] \\
& =\frac{\Omega_{x} x+\Omega_{y} Y}{\sqrt{x^{2}+y^{2}}} .
\end{aligned}
$$

Equation ( $B-12$ ) along with Equation ( $B-10)$ provides the transformation for the direction vector $\bar{\Omega}$ from the rectangular coordinate system to the cylindrical coordinate system. 
APPENDIX C

COMPARISON OF $x$ * AND W

In this appendix, a comparison of the point value $x^{*}$ and the event value $W$ is presented. Three particle trajectories are chosen, and their importance functions in different regions along the trajectories will be discussed. All three trajectories are for neutrons of group one energy and are shown in Figure 18. The corresponding $x^{*}$ and $W$ in different regions are presented in Table 8.

It can be seen from Table 8 . that in the same region $W$ is smaller than $x^{\star}$ by one to two order of magnitude. This is not surprising because the value of a particle entering a collision is reduced by the absorption probability and the collision process before the particle emerges with the same energy group and direction. The relative $x^{*}$ and $W$ in Table 8 were obtained by dividing the importance functions in different regions by the importance of the region where the trajectory originated. From trajectories I and II, it can be seen that the $W$ is a stronger importance function than $x^{*}$. Hence, $W$ would encourage more deep penetration than $x^{*}$, particularly for trajectory I. Finally, for trajectory III, W suggests that regions 7 and $7^{-}$are about a thousand times more important than regions 1 and $1 \%$. However, $x^{*}$ suggests that the value of a particle approaching the duct is roughly ten times of the value leaving the duct. 


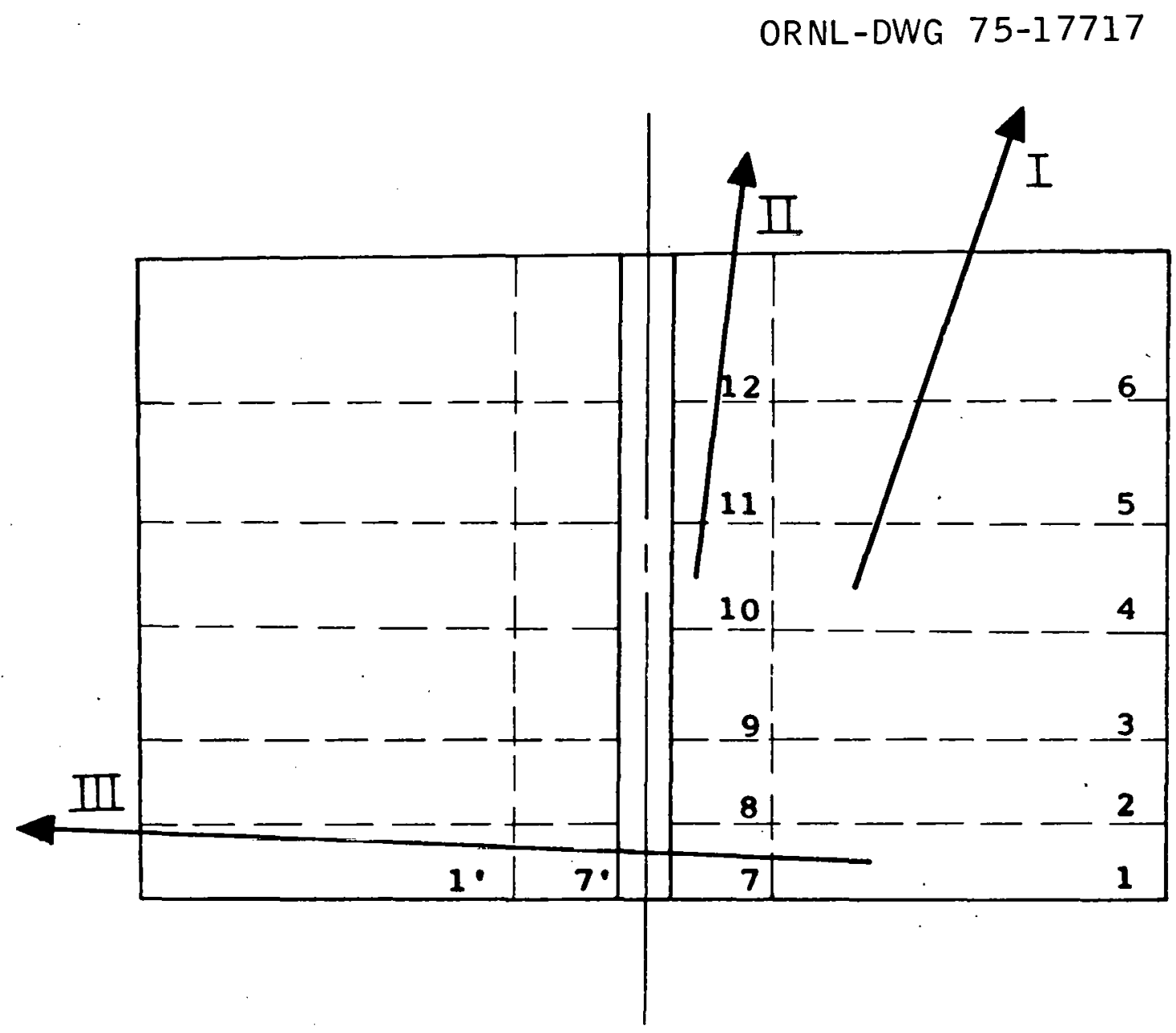

Figure 18. Particle Trajectories in the Geometry of the Standard Problem. 
108

TABLE 8

COMPARISON OF $x *$ AND $w^{\mathrm{a}}$

\begin{tabular}{|c|c|c|c|c|c|}
\hline \multicolumn{2}{|c|}{ Trajectory } & \multirow{2}{*}{$\frac{x^{\star}}{5.41-8^{b}}$} & \multirow{2}{*}{$\frac{x_{\text {RELATIVE }}^{\star}}{1}$} & \multirow{2}{*}{$\frac{W}{2.41-10}$} & \multirow{2}{*}{$\frac{W_{\text {RELATIVE }}}{1}$} \\
\hline I. & Region 4 & & & & \\
\hline & Region 5 & $4.83-7$ & 8.93 & $3.73-9$ & $1.55+1$ \\
\hline & Region 6 & $2.59-6$ & $4.79+1$ & $8.35-8$ & $3.46+2$ \\
\hline \multirow[t]{3}{*}{ I I. } & Region 10 & $1.72-7$ & 1 & $1.24-9$ & 1 \\
\hline & Region 11 & $1.05-6$ & 6.10 & $9.30-9$ & 7.50 \\
\hline & Region 12 & $5.47-6$ & $3.18+1$ & $1.05-7$ & $8.47+1$ \\
\hline \multirow[t]{4}{*}{ I I . } & Region 1 & $1.37-10$ & 1 & $1.17-12$ & 1 \\
\hline & Region 7 & $4.29-9$ & $3.13+1$ & $1.46-10$ & $1.25+2$ \\
\hline & Region $7^{-}$ & $6.42-10$ & 4.69 & $2.20-10$ & $1.38+2$ \\
\hline & Region $1^{-}$ & $1.38-11$ & 0.10 & $3.40-12$ & 2.91 \\
\hline
\end{tabular}

$a^{*}$ and $w$ have the dimensions of response/neutron.

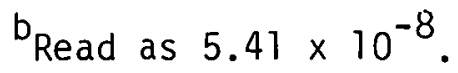


APPENDIX D

\section{PLOTS OF ADJOINT DOT CALCULATIONS}

The plots of adjoint fluxes of the adjoint calculations with axial adjoint source and off-axial adjoint source are presented in this appendix. Figures 19 through 26 are adjoint flux plots from the adjoint DOT calculation with axial adjoint source, and Figures 27 through 34 are adjoint flux plots from the adjoint DOT calculation with off-axial adjoint source. 


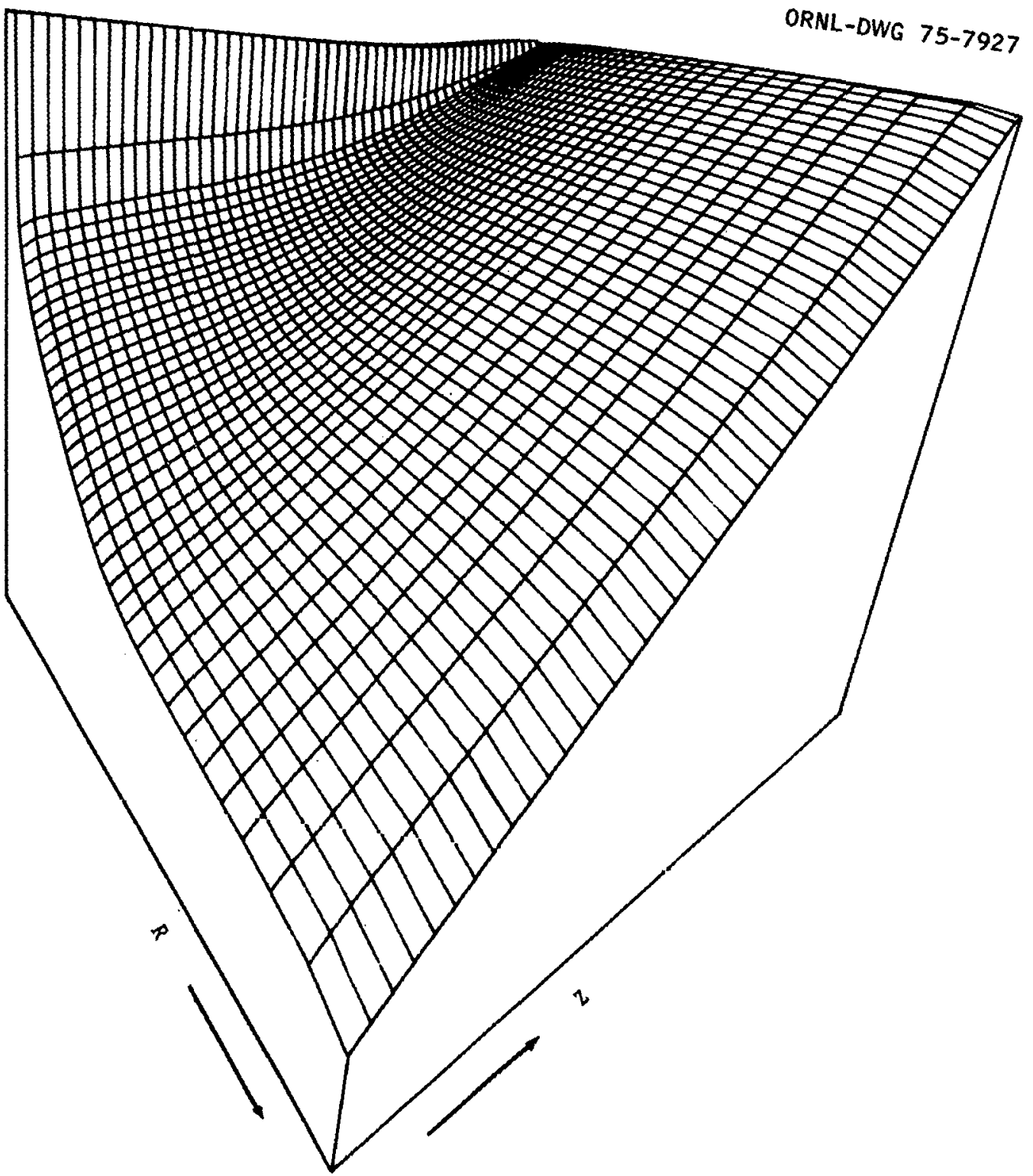

Figure 19.

$$
\begin{aligned}
& \text { Total Adjoint Flux Contour of the DOT Calculation with } \\
& \text { Axial Adjoint Source. }
\end{aligned}
$$




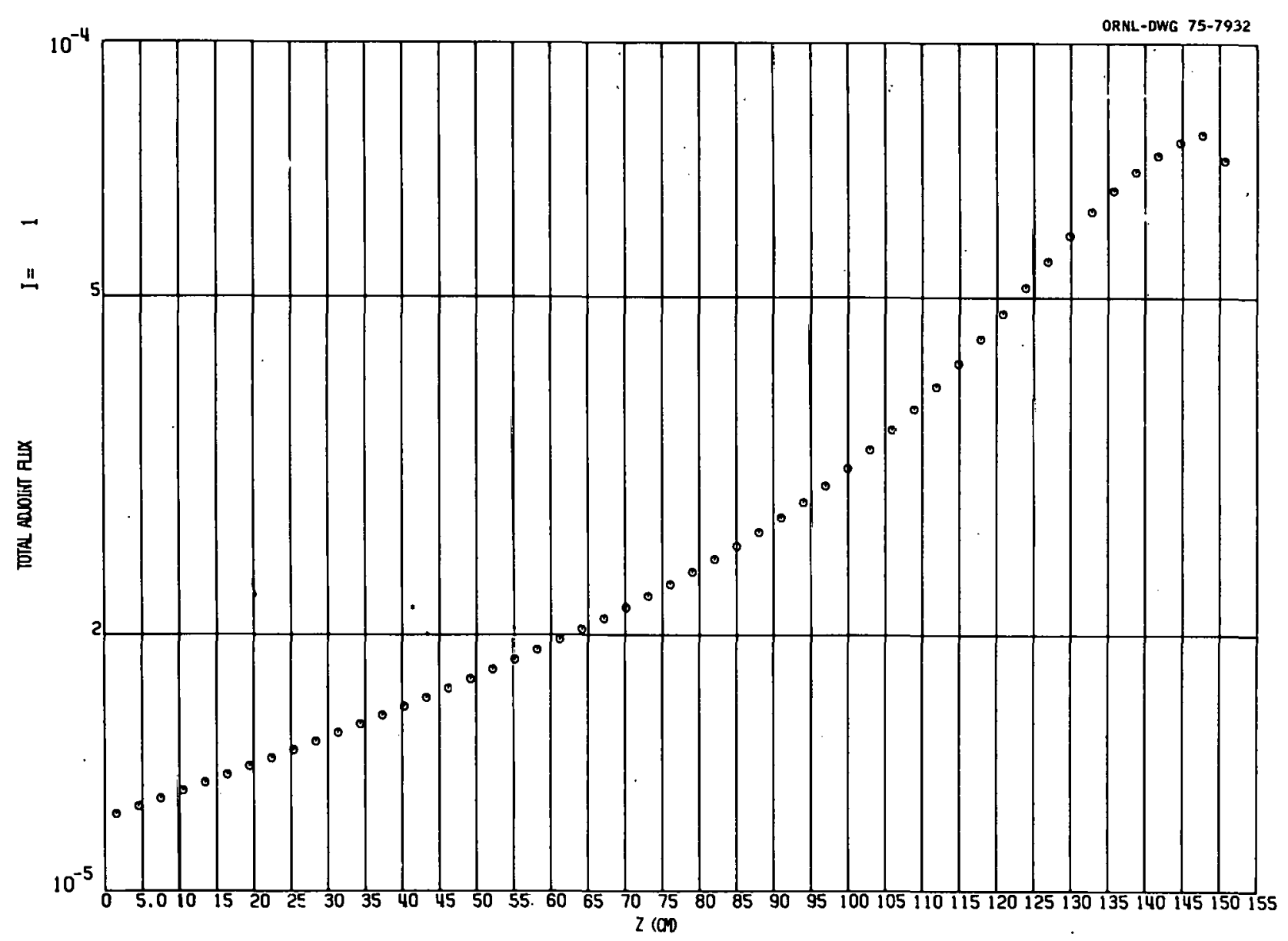

Figure 20. Total Adjoint Flux Versus Z-Axis in the Duct of the DOT Calculation with Axial Adjoint Source. 


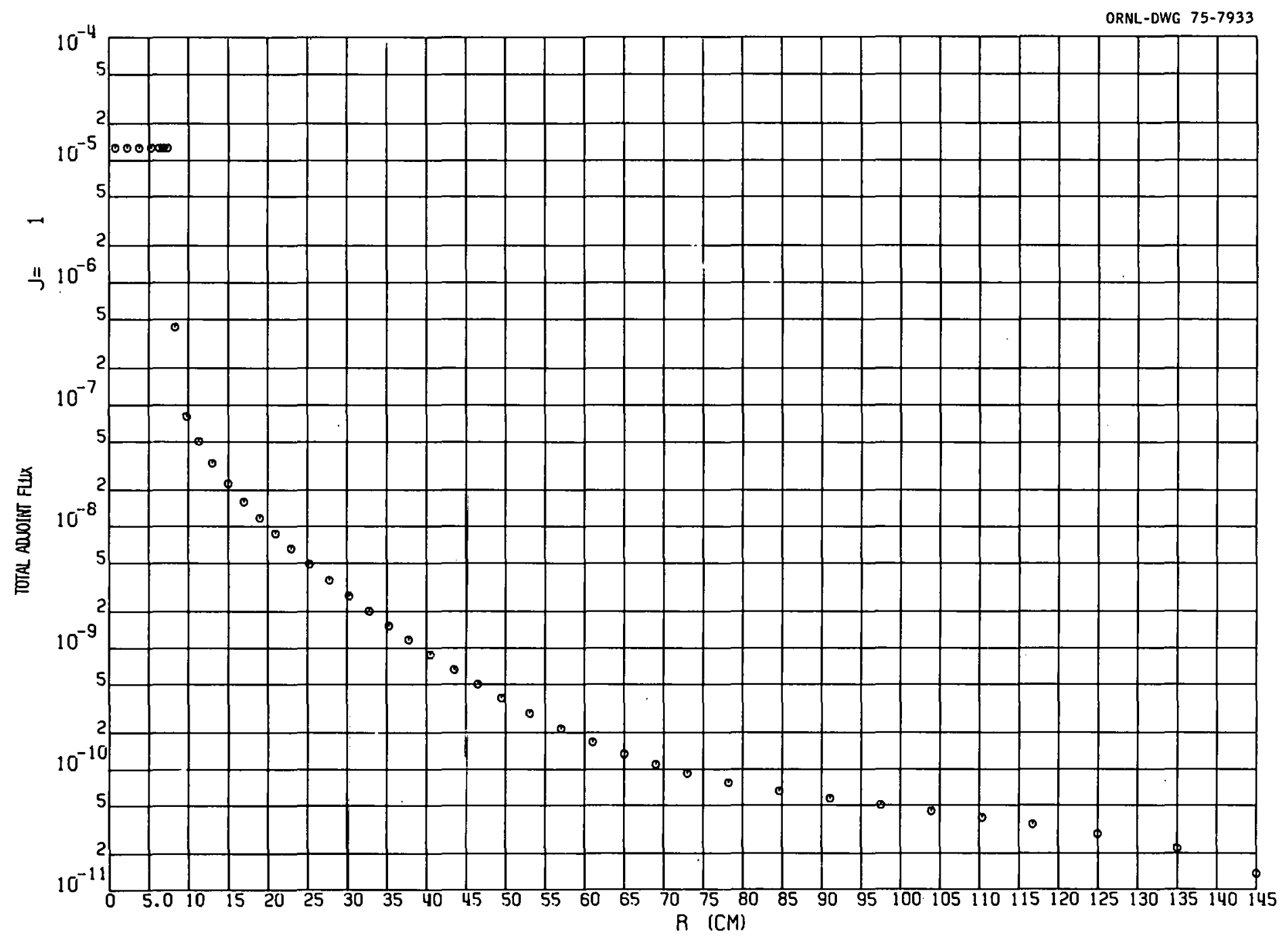

Figure 21. Total Adjoint Flux Versus Radius at the Bottom Surface of the Cylinder of the DOT Calculatior with Axial Adjoint Source. 


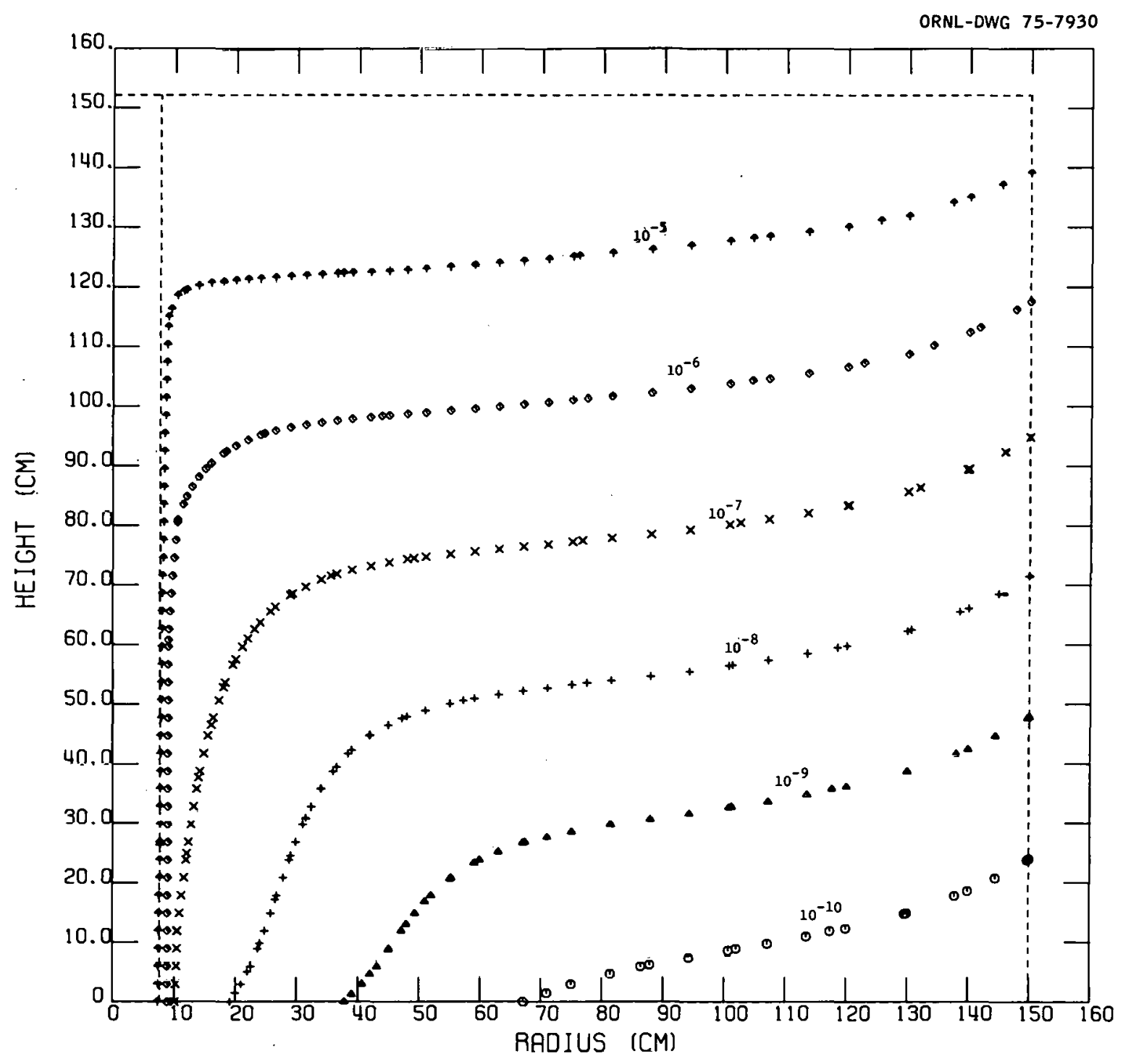

Figure 22. Total Adjoint Flux Levels of the DOT Calculation with Axial Adjoint Source. 


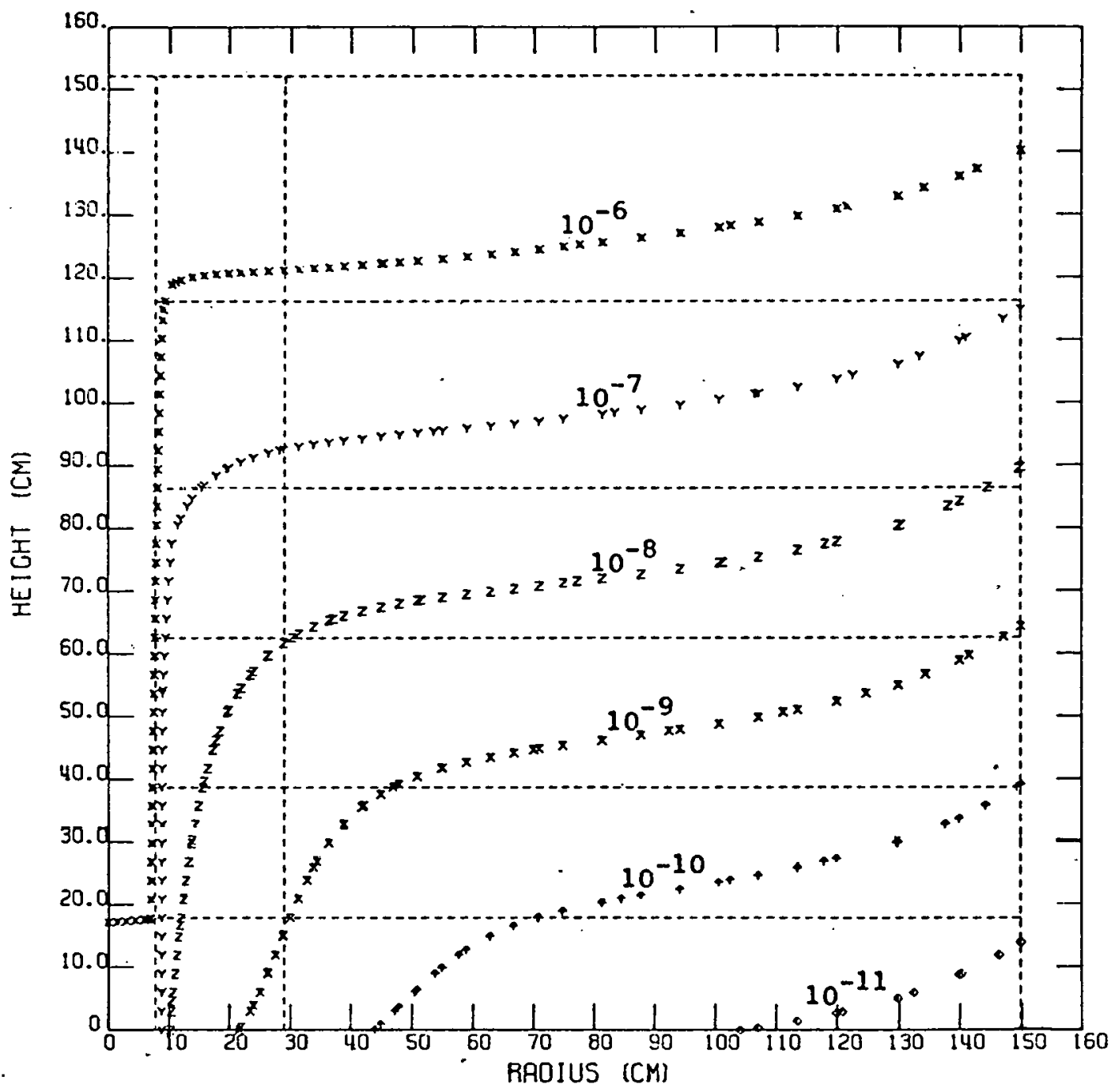

Figure 23. Group 1 Adjoint Flux Levels of the DOT Calculation with Axial Adjoint Source. 


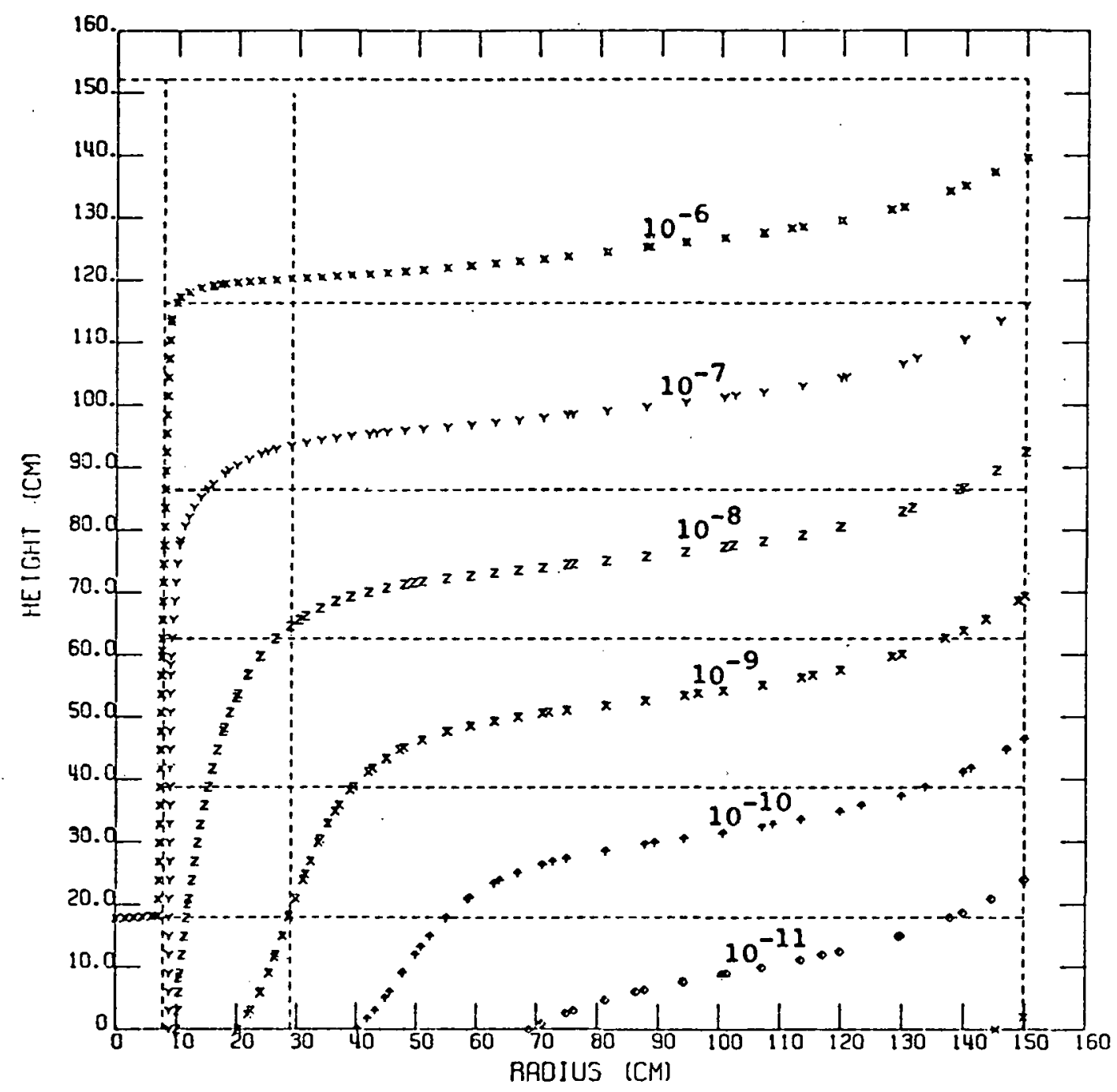

Figure 24. Group 5 Adjoint Flux Levels of the DOT Calculation with Axial Adjoint Source. 


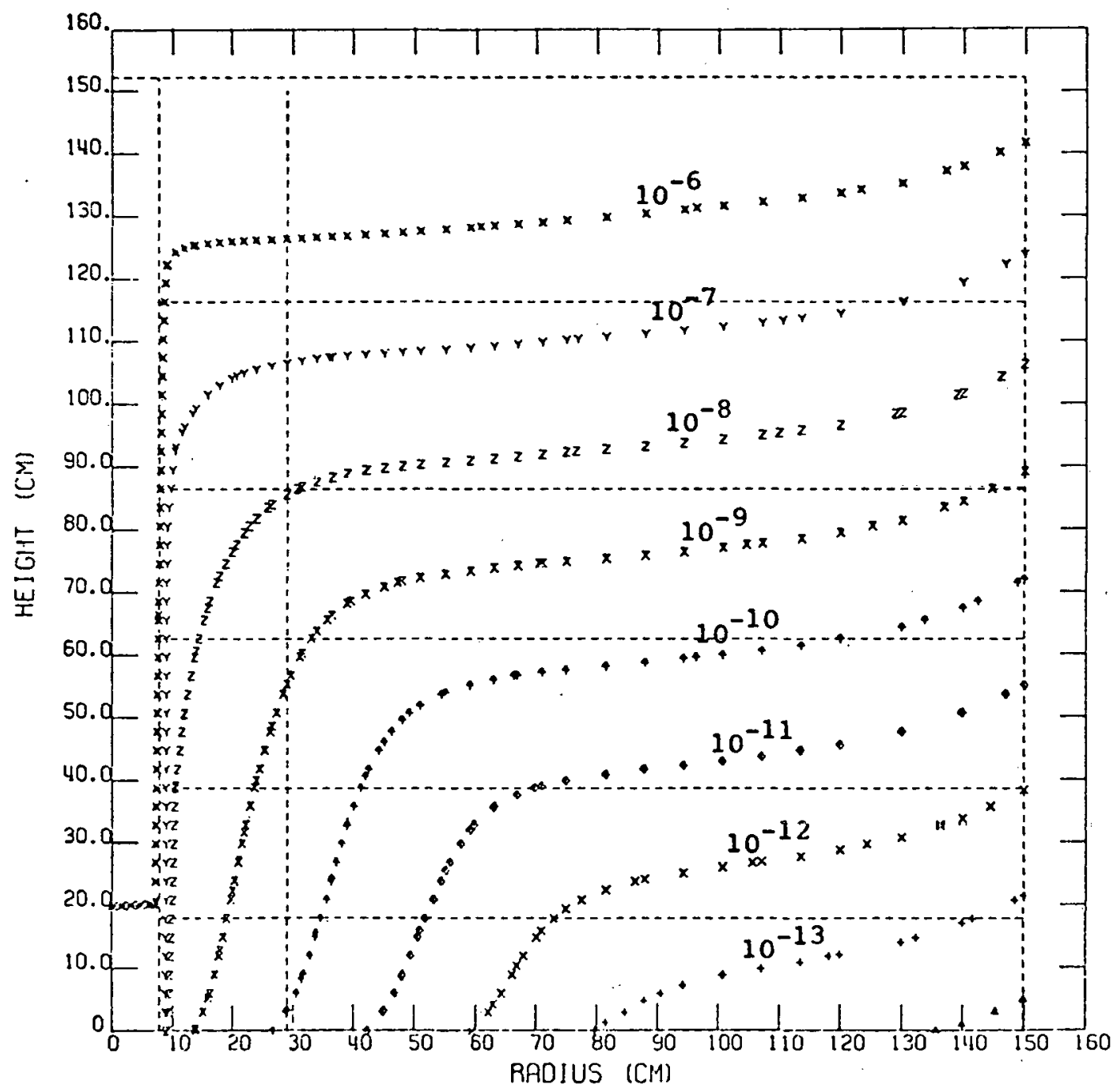

Figure 25. Group 10 Adjoint Flux Levels of the DOT Calculation with Axial Adjoint Source. 


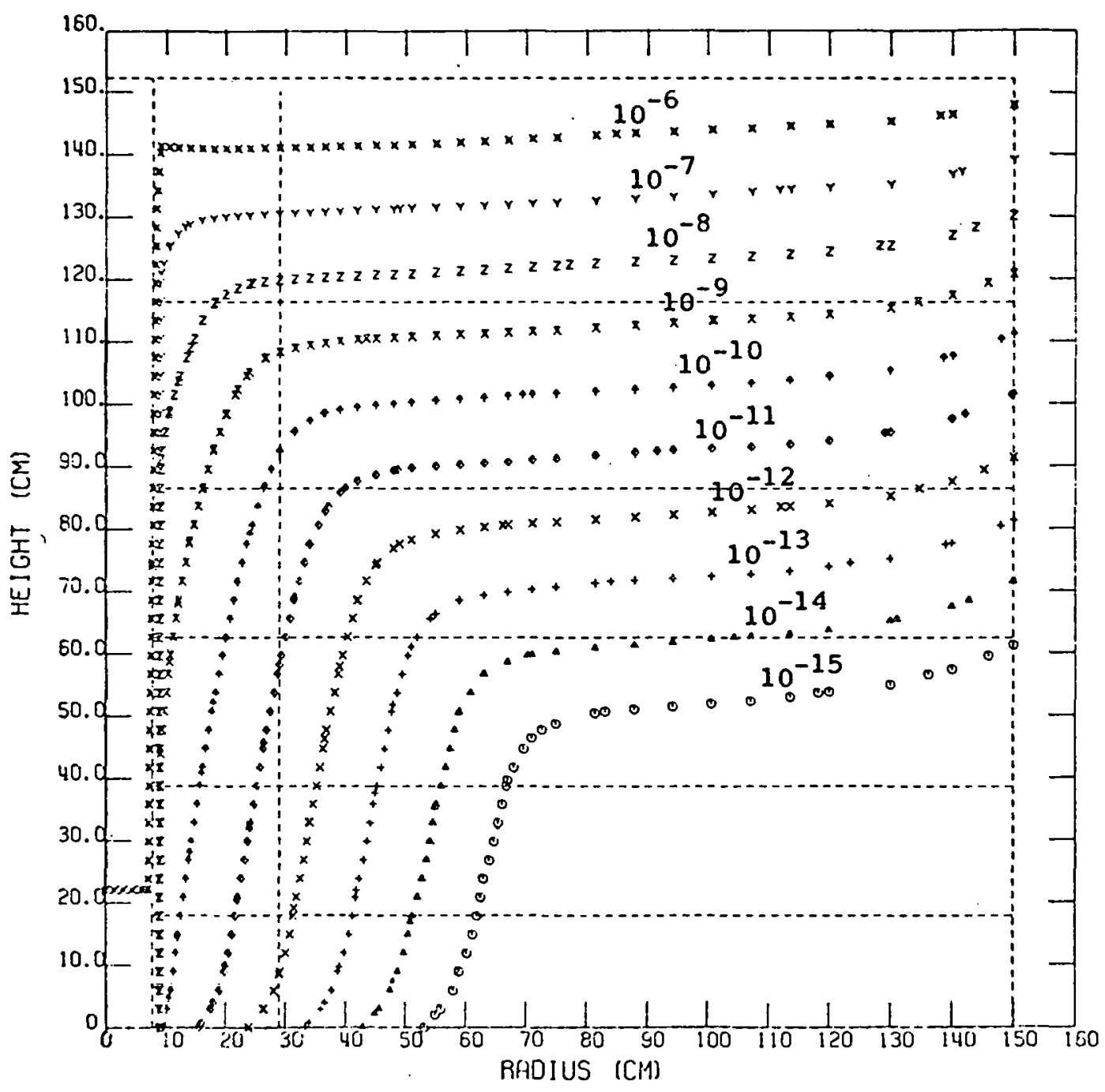

Figure 26. Group 14 Adjoint Flux Levels of the DOT Calculation with Axial Adjoint Source. 


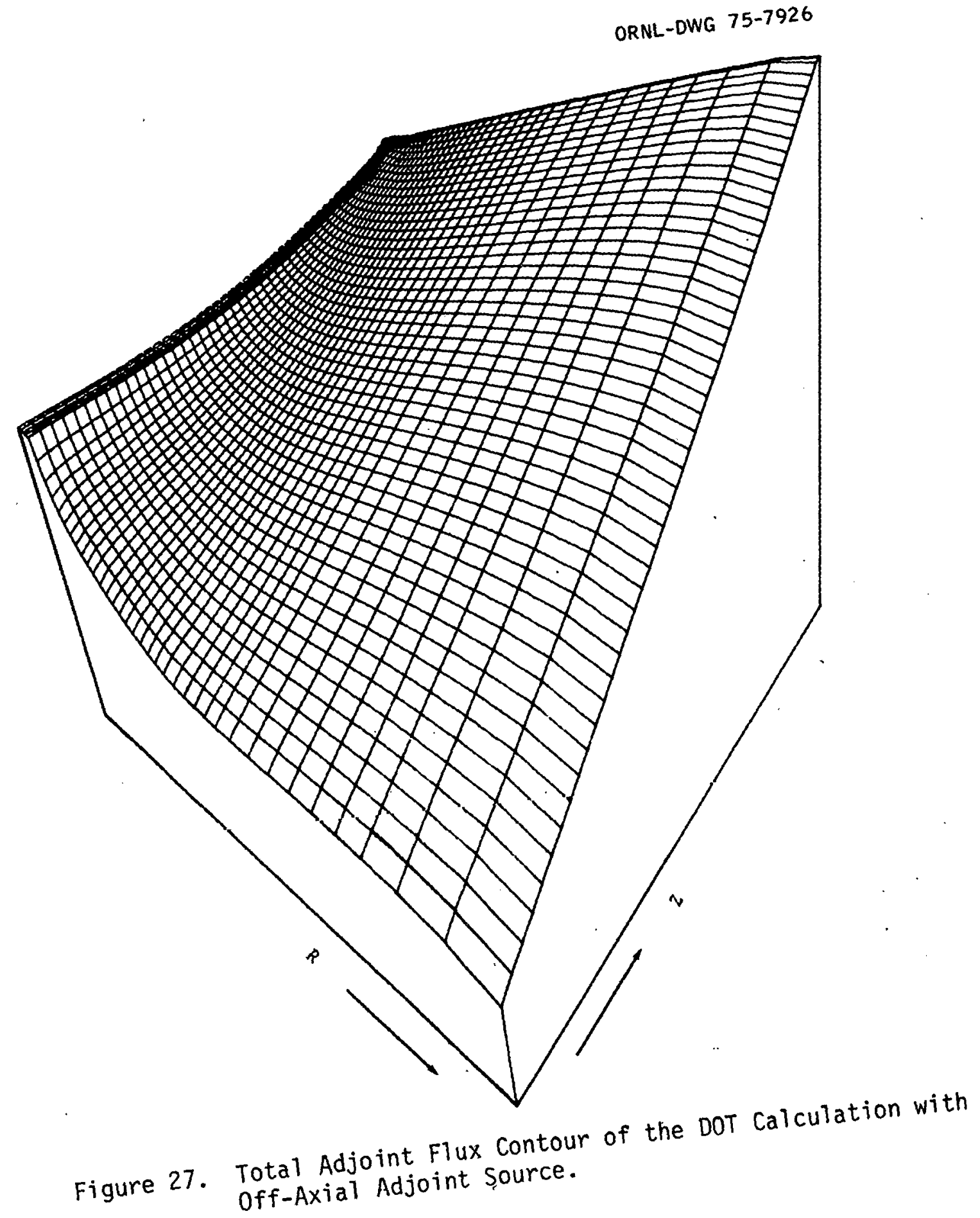




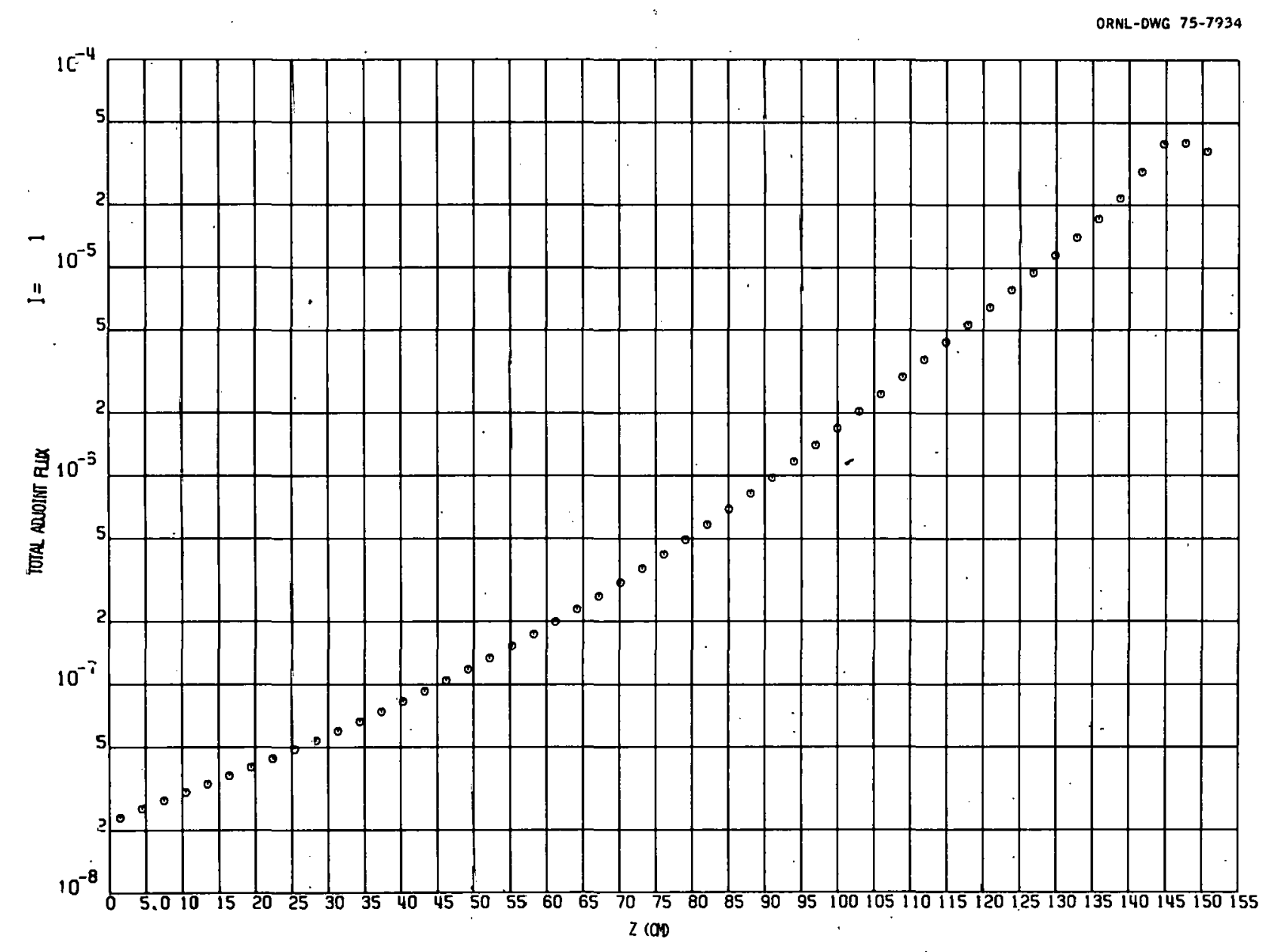

Figure 28. Total Adjoint Flux Versus:Z-Axis in the Duct of the DOT Calculation with Oïf-Axial Adjoint Source. 


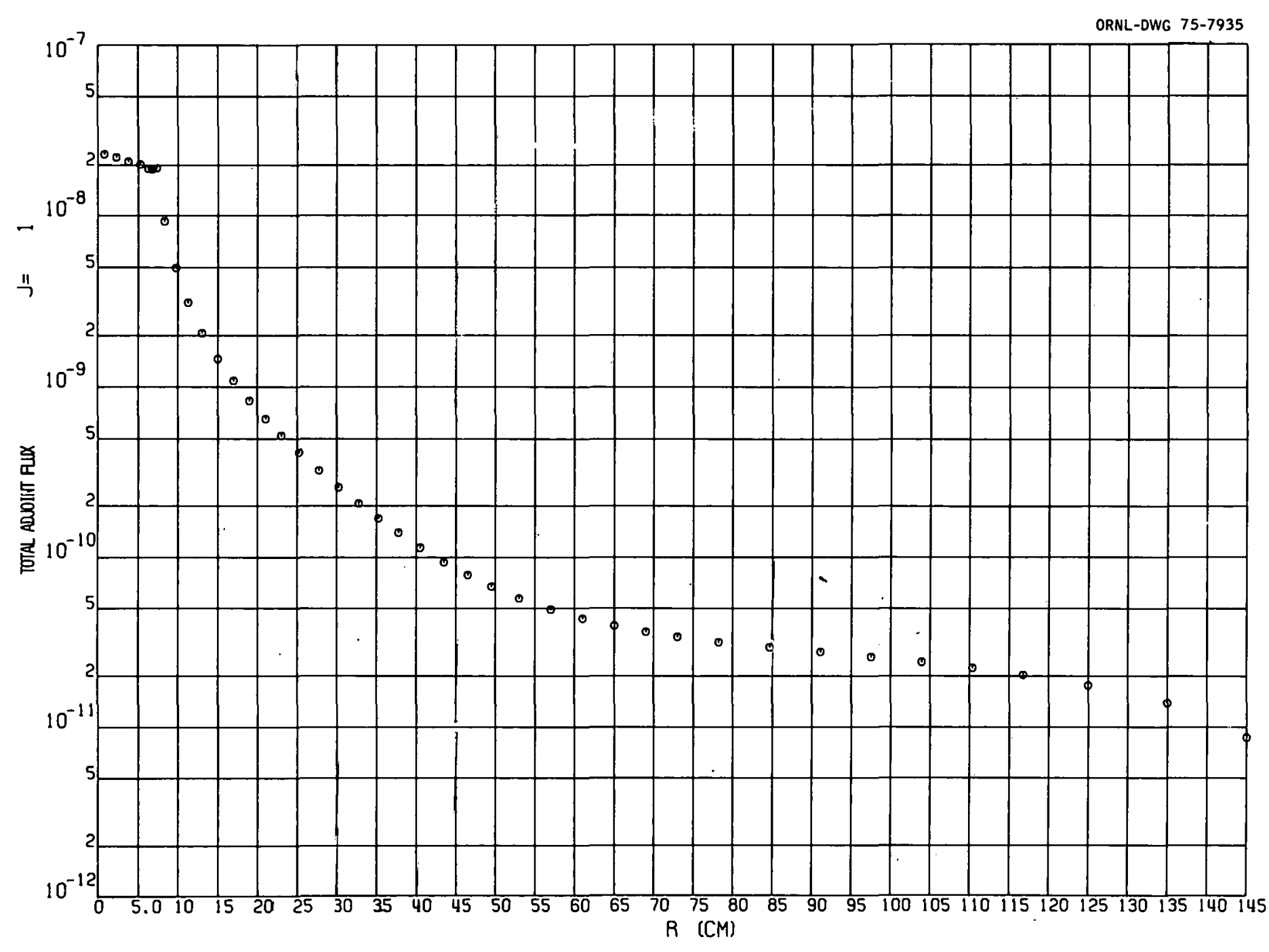

Figure 29. Total Adjoint Flux Versus Radius at the Bottom Surface of the Cylinder of the DOT Calculation with Off-Axial Adjoint Source. 


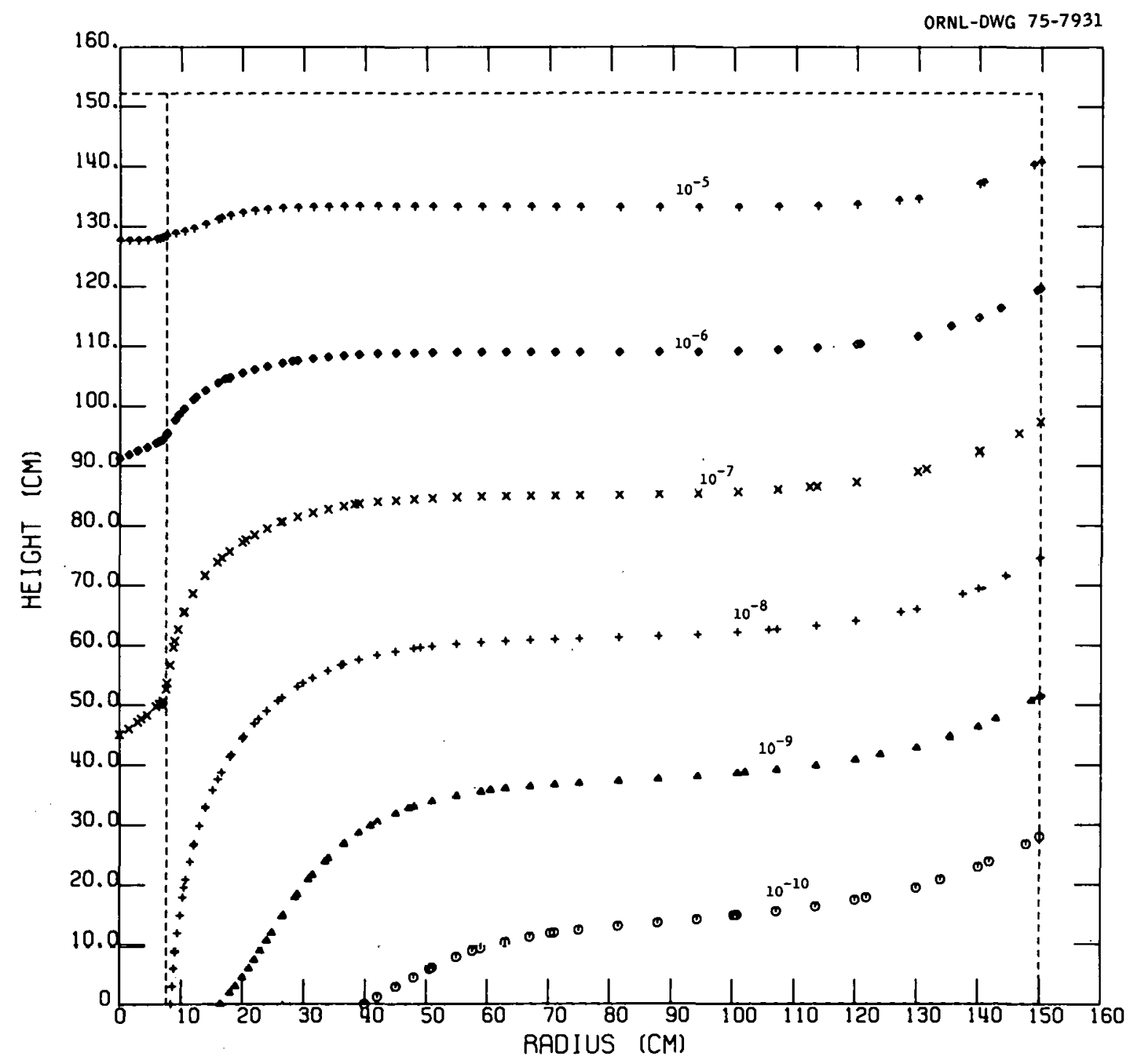

Figure 30. Total Adjoint Flux Levels of the DOT Calculation with OffAxial Adjoint Source. 


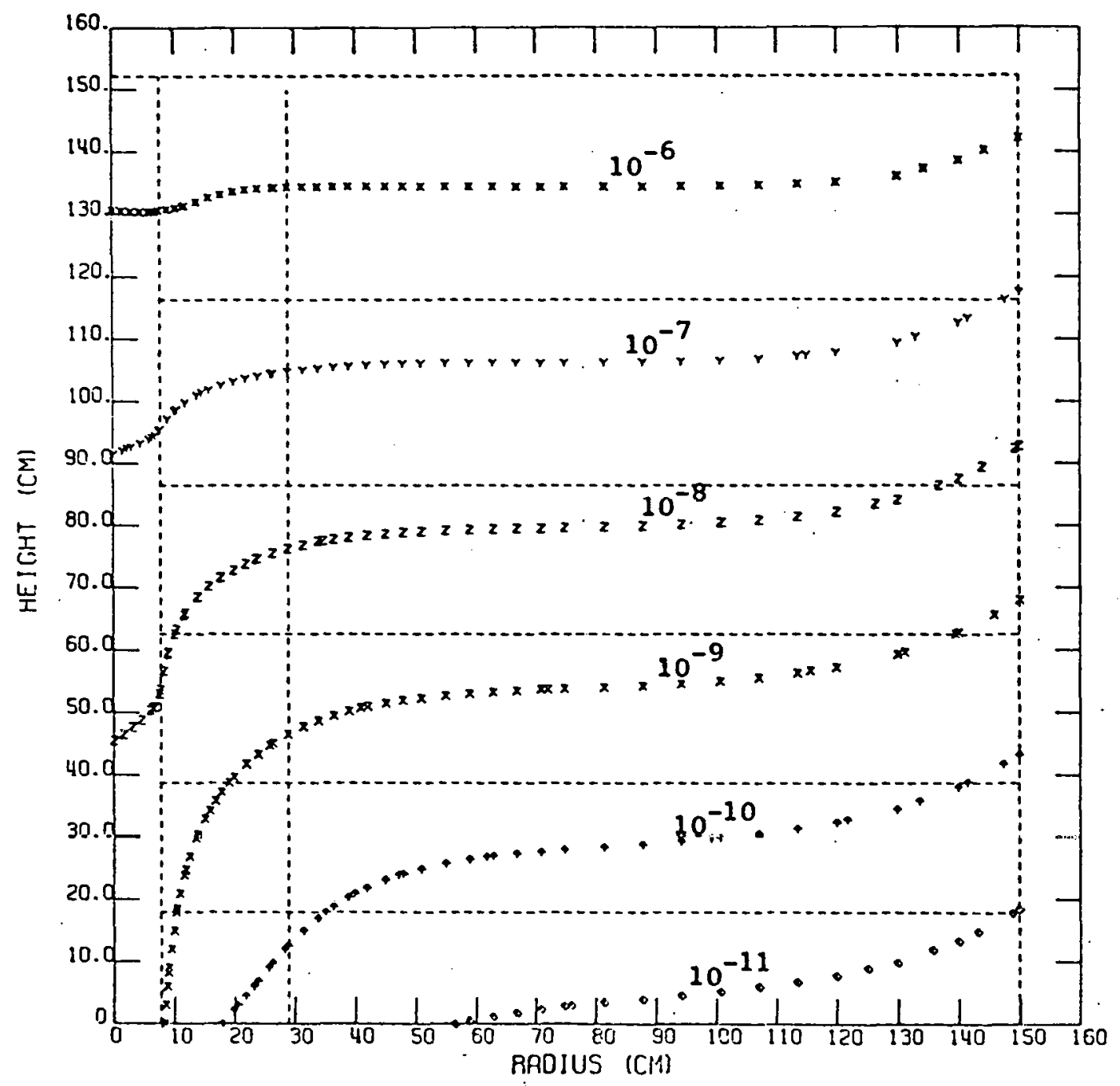

Figure 31. Group 1 Adjoint Flux Levels of the DOT Calculation with Off-Axial Adjoint Source. 


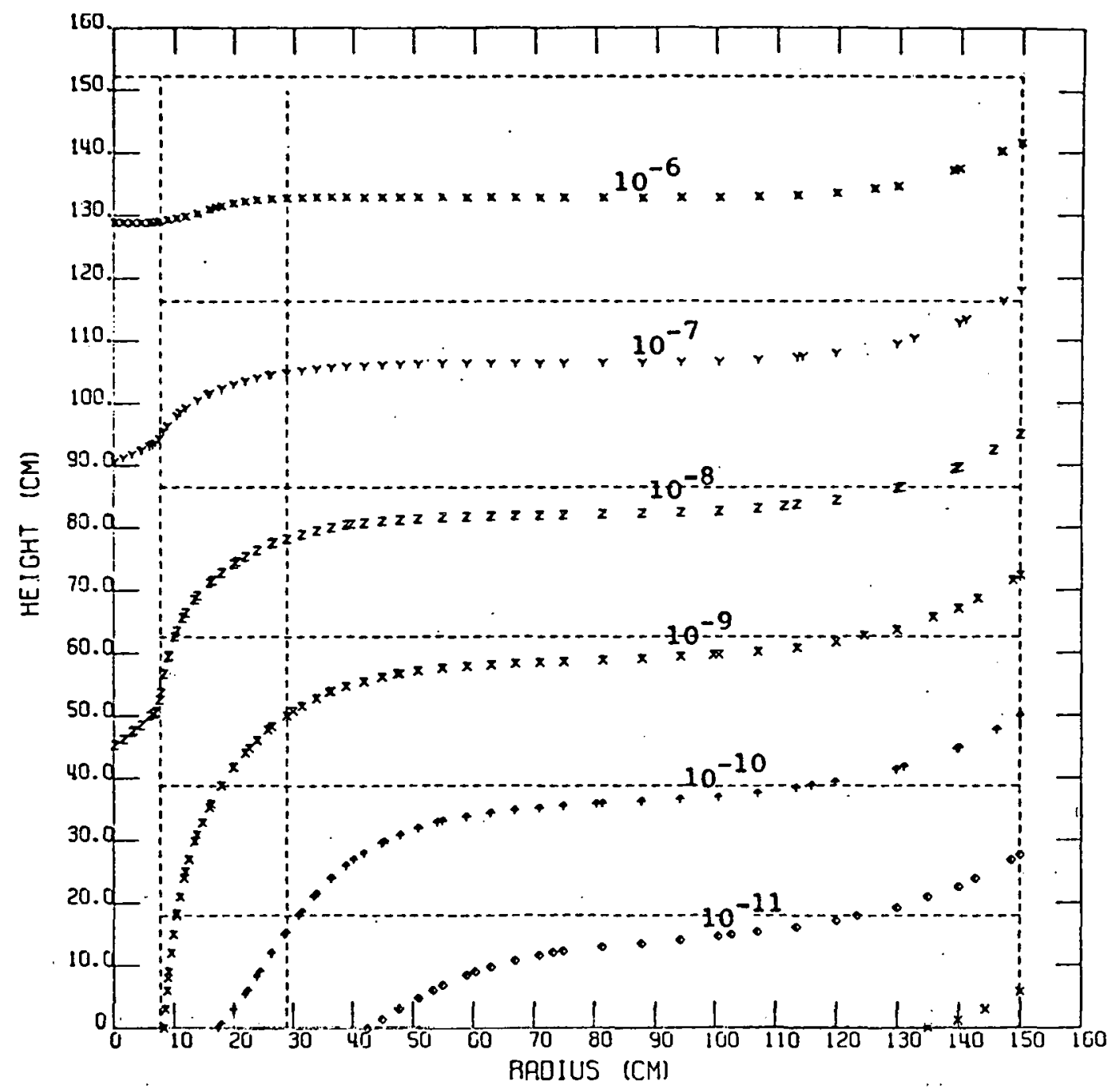

Figure 32: Group 5 Adjoint Flux Levels of the DOT Calculation with Off-Axial Adjoint Source. 


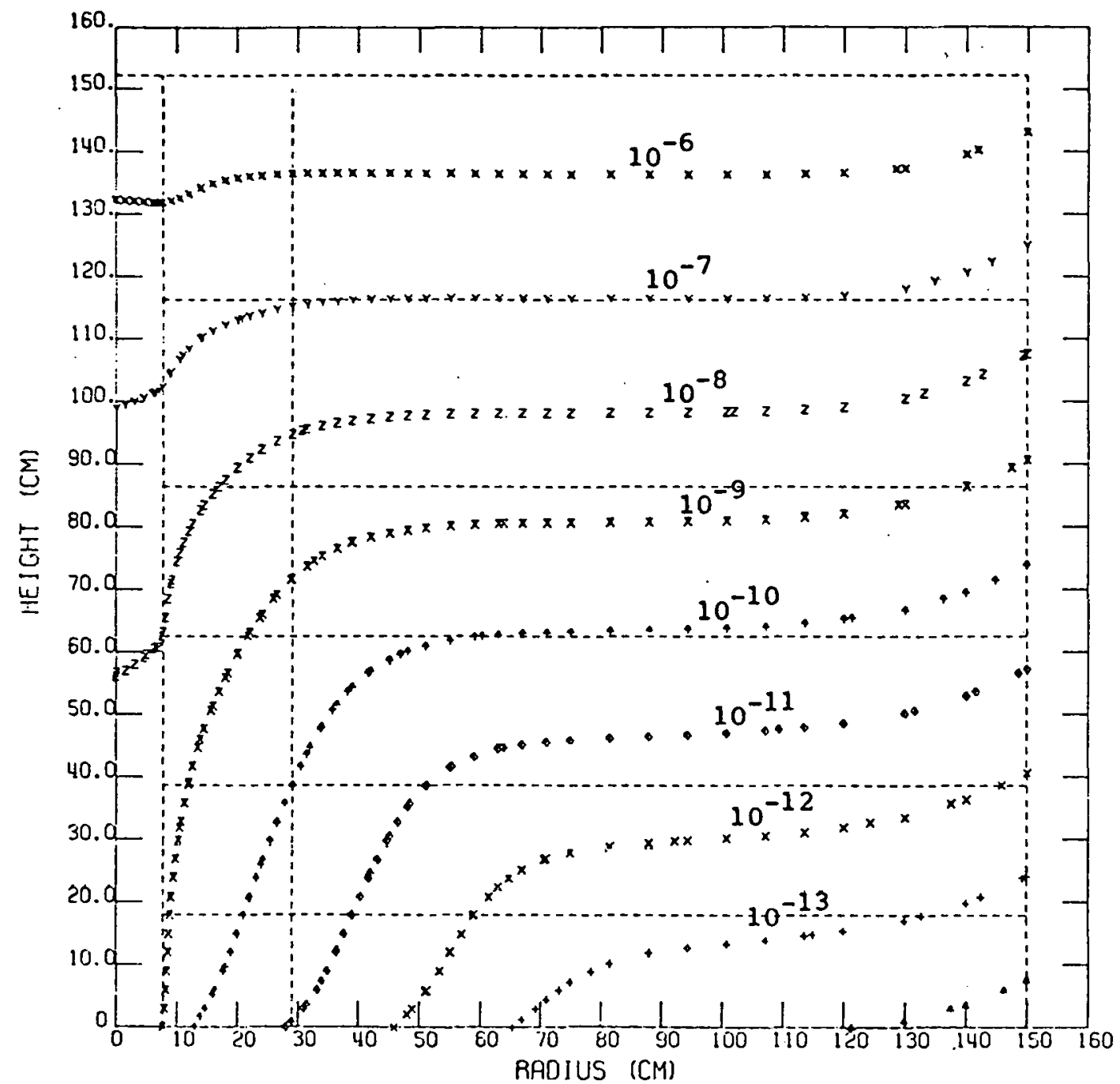

Figure 33. Group 10 Adjoint Flux Levels of the DOT Calculation. with Off-Axial Adjoint Source. 


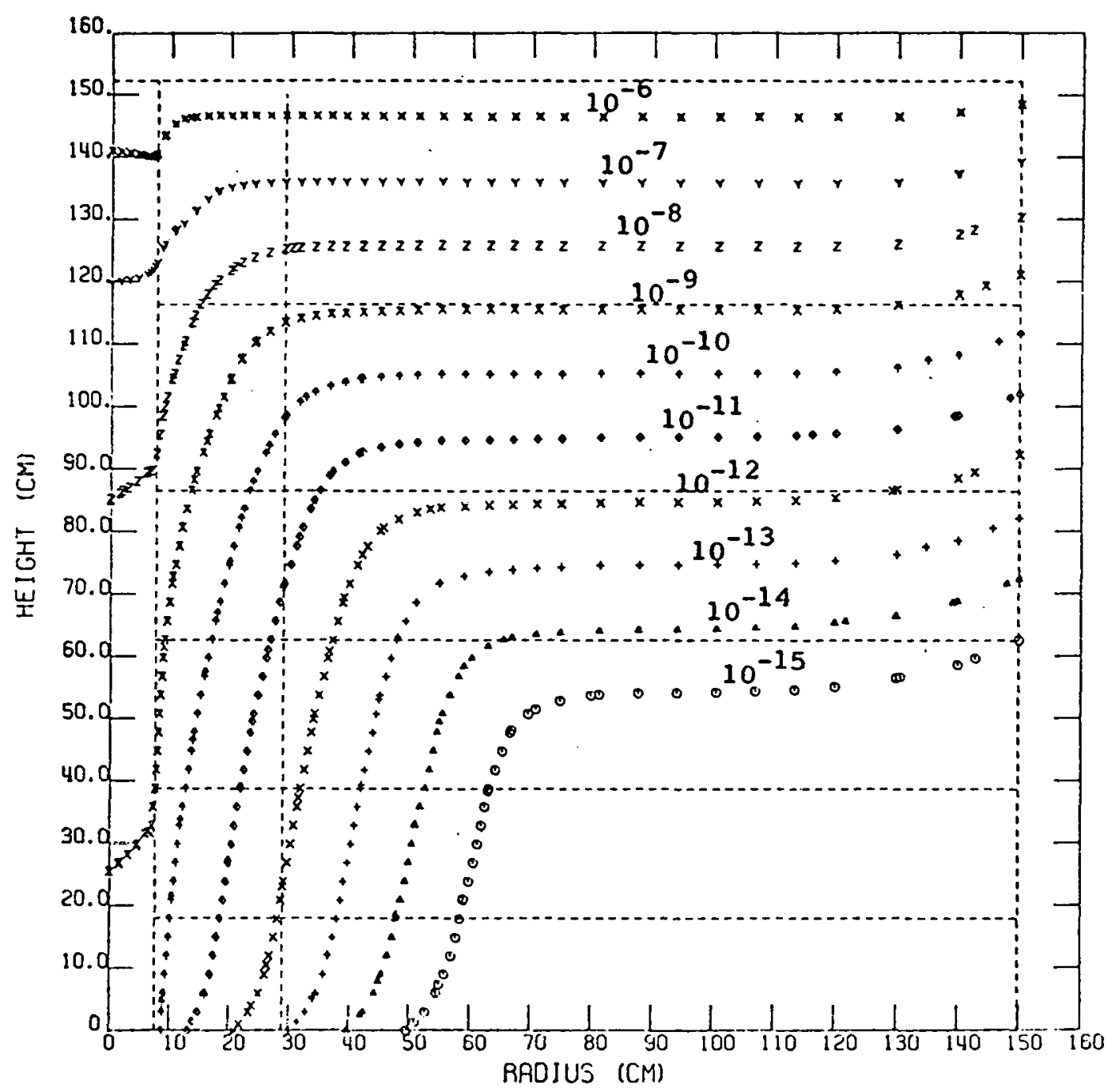

Figure 34. Group 14 Adjoint Flux Levels of the DOT Calculation with Off-Axial Adjoint Source. 


\section{APPENDIX E}

\section{NEUTRON CROSS SECTION FOR CONCRETE}

This appendix contains the 22 group $P_{3}$ neutron cross section set for the concrete used in the standard problem. The compositions of the concrete are presented in Table 9 along with the percentage of the atomic density for each 'element. 
TABLE 9

CONCRETE COMPOSITION ${ }^{\mathrm{a}}$

\begin{tabular}{ccc}
\hline \hline Element & Composition (atom/b-cm) & Atomic Density in Percent \\
\hline$H$ & $1.065(-2)^{\mathrm{b}}$ & $13.71 \%$ \\
$\mathrm{C}$ & $1.310(-4)$ & $0.17 \%$ \\
$\mathrm{O}$ & $4.084(-2)$ & $52.58 \%$ \\
$\mathrm{Na}$ & $1.071(-3)$ & $1.38 \%$ \\
$\mathrm{Mg}$ & $1.620(-4)$ & $0.21 \%$ \\
$\mathrm{Al}$ & $2.822(-3)$ & $3.63 \%$ \\
$\mathrm{Si}$ & $1.332(-2)$ & $17.15 \%$ \\
$\mathrm{~K}$ & $8.280(-4)$ & $1.07 \%$ \\
$\mathrm{Ca}$ & $2.426(-3)$ & $3.12 \%$ \\
$\mathrm{Fe}$ & $5.428(-3)$ & $6.99 \%$ \\
\hline & \\
\hline
\end{tabular}


CROSS SECTIONS AS READ FOR CONCRETE

\begin{tabular}{|c|c|c|}
\hline $\begin{array}{l}8 \\
1 \\
2 \\
3 \\
4 \\
5 \\
6 \\
7 \\
8\end{array}$ & $\begin{array}{l}A B S \times S E C \\
1.642 E-C 2 \\
1.961 E-02 \\
1.601 E-02 \\
1.125 E-02 \\
5.024 E-03 \\
4.848 E-03 \\
1.624 E-03 \\
5.522 E-C 4\end{array}$ & $\begin{array}{l}\text { NU.F ISS } \\
0.0 \\
0.0 \\
0.0 \\
0.0 \\
0.0 \\
0.0 \\
0.0 \\
0.0\end{array}$ \\
\hline 9 & $3.315 E-04$ & 0.0 \\
\hline 10 & $1.894 E-04$ & 0.0 \\
\hline 11 & $0.530 E-05$ & 0.0 \\
\hline 17 & $4.895 E-05$ & 0.0 \\
\hline 13 & $5.700 E-05$ & 0.0 \\
\hline 14 & $2.392 E-04$ & 0.0 \\
\hline 15 & $1.019 E-03$ & 0.0 \\
\hline 16 & $2.575 E-04$ & 0.0 \\
\hline 17 & $5.233 E-04$ & 0.0 \\
\hline 13 & 9.08 IE -04 & 0.0 \\
\hline 19 & $1.595 E-03$ & 0.0 \\
\hline 20 & $2.779 E-03$ & 0.0 \\
\hline 21 & $4.574 E-03$ & 0.0 \\
\hline 2 & $2.005 E-02$ & 0.0 \\
\hline
\end{tabular}

$1.5916-01$

$1.5005-01$

$1.688 E-01$

2. $19 B E-01$

2. $B 2.3 E-01$

3. $751 E-01$

4. $231 E-01$

4. $946 E-01$

$4.734 E-01$

4. $763 E-01$

$4.768 E-01$

4. $777 E-01$

$4.7925-01$

$4.813 E-01$

4. $973 E-01$
COEFFICIENT $\&\left(\mathrm{P}_{0}\right)$

TRANSFER PROBABILIIIES

$5.867 E-02$

$\begin{array}{lll}5.465 E-02 & 7.829 E-03\end{array}$

$4.674 E-02 \quad 1.789 E-02 \quad 2.352 E-03$

$4.697 E-02 \quad 2.182 E-02 \quad 3.450 E-03 \quad 6.510 E-03$

$6.820 E-02 \quad 2.934 E-02 \quad 3.591 E-03 \quad 2.955 E-03 \quad 5.891 E-03$

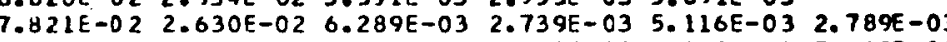

$1.195 E-01 \quad 4.083 E-02 \quad 1.241 E-02 \quad 4.561 E-03 \quad 4.362 E-03 \quad 7.462 E-03 \quad 5.447 E-03$

7.051E-02 4.357E-02 $8.173 E-03 \quad 4.092 E-03 \quad 2.849 E-03 \quad 4.303 E-03 \quad 2.811 E-03 \quad 4.032 E-C 3$

3.103E-02 1.875E-02 2.280E-03 2.172E-03 7.433E-04 7.248E-04 1.019E-03 7.504E-04 $1.111 \mathrm{E}-03$

9.0I3E-02 5.520E-02 2.891E-02 7.15CE-C3 7.203E-03 3.855E-03 3.231E-03 $5.788 E-03$ $2.8 T 9 E-03 \quad 4.400 E-03$

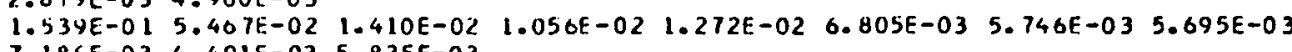
$7.186 E-03 \quad 4.491 E-03 \quad 5.835 E-03$

$2.159 E-01 \quad 4.900 E-0 ?$ ? $1.040 F-02 \quad 9.223 E-03 \quad 1.410 E-02 \quad 6.426 E-03 \quad 5.583 E-03 \quad 4.521 E-03$ $4.3112 E-03 \quad 3.856 E-03 \quad 3.378 E-03 \quad 4.31,4 E-C 3$ $3.199 \mathrm{E}-03 \quad 3.551 \mathrm{E}-03 \quad 1.834 \mathrm{E}-03 \quad 1.866 \mathrm{E}-03 \quad 2.723 \mathrm{E}-03$ $1.212 E-04$ 6. $289 E-045.412 E-042.118 E-042.075 E-042.872 E-04$

1.77E-01 4.792E-02 $1.228 E-032.022 E-04$ 7.455E-05 4.007E-05 3.211E-05 2.672E-05 $1.813 \mathrm{E}-05 \quad 1.897 \mathrm{E}-05$ 1.522E-05 $1.21 \mathrm{lE}-05 \quad 4.393 \mathrm{E}-06 \quad 3.897 \mathrm{E}-06 \quad 4.061 \mathrm{EE}-06$

(1) 4. B.3 $1.030=-07$.

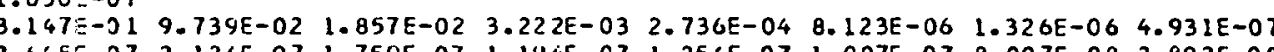

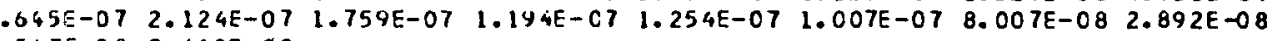
$2.547 E-08 \quad 2.610 E-08$

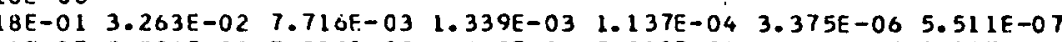
$2.049 E-07 \quad 1.099 E-07 \quad 8.826 E-08 \quad 7.309 E-C 8$ 4. $960 E-08$ 5.212E-08 4. $183 E-08$ 3.327E-0B 1.202E-08 $1.058 E-08$ $1.085 E-0 B$

4. $281 F-03 \quad 1.958 E-03 \quad 3.398 E-04 \quad 2.885 E-05 \quad 8.565 E-07$

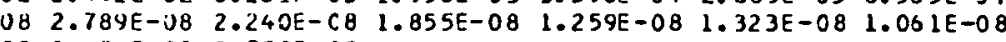
(1.

2.886E-02 $9.163 E-03 \quad 3.044 E-03 \quad 7.199 E-04 \quad 1.249 E-04 \quad 1.061 E-05$ $\begin{array}{lllll} & \end{array}$ (.121E-09 $9.874 E-101.012 E-09$

$\begin{array}{lllll} & \end{array}$ 2.993F-0ن $\quad$ 1.113E-CB 5. $569 E-09$ 4. $794 E-09$ 3.970E-09 $2.694 E-09$ $2.830 E-0 y \quad 2.272 E-09$ 1.807F-09 6.527E-10 5.748E-10 5.891E-10

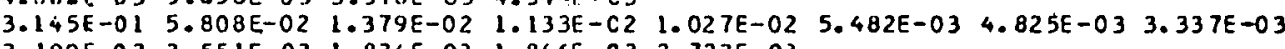

$\begin{array}{llllllll}3.562 F-01 & 5.903 E-02 & 7.973 E-03 & 2.937 F-03 & 2.032 E-03 & 1.376 F-03 & 1.132 E-03 & 8.896 E-04\end{array}$ 
CROSS SECTIONS AS READ FOR CONCRETE

\begin{tabular}{|c|c|c|c|}
\hline $\begin{array}{l}3 \\
1 \\
2 \\
3 \\
4 \\
5 \\
6 \\
7 \\
8\end{array}$ & $\begin{array}{l}\text { AB.5 XSEC } \\
0.0 \\
0.0 \\
0.0 \\
0.0 \\
0.0 \\
0.0 \\
0.0 \\
0.0\end{array}$ & $\begin{array}{l}\text { NU*F ISS } \\
0.0 \\
0.0 \\
0.0 \\
0.0 \\
0.0 \\
0.0 \\
0.0 \\
0.0\end{array}$ & $\begin{array}{l}\text { TOTAL } \\
1.267 E-01 \\
1.274 E-01 \\
1.195 E-01 \\
1.199 E-01 \\
1.347 E-01 \\
1.579 E-C 1 \\
1.991 E-01 \\
1.500 E-01\end{array}$ \\
\hline 9 & 0.0 & 0.0 & $1.216 E-D 1$ \\
\hline 10 & 0.0 & 0.0 & $1.68 \mathrm{AE}-\mathrm{DI}$ \\
\hline 11 & 0.0 & 0.0 & 2. $198 \mathrm{~F}-01$ \\
\hline 12 & 0.0 & 0.0 & $2.823 E-32$ \\
\hline 13 & 0.0 & 0.0 & $3.751 E-0.1$ \\
\hline 14 & 0.0 & 0.0 & $4.23 L E-01$ \\
\hline 15 & 0.0 & 0.0 & $4.96,6 E-01$ \\
\hline 16 & 0.0 & 0.0 & 4. $734 E-01$ \\
\hline 17 & 0.0 & 0.0 & $4.763 E-C 1$ \\
\hline 18 & 0.0 & 0.0 & $4.768 E-C .1$ \\
\hline 19 & 0.0 & 0.0 & 4.7TIE-CI \\
\hline 20 & 0.0 & 0.0 & 4. $792 E-O L$ \\
\hline 21 & 0.0 & 0.0 & $4.813 E-0.1$ \\
\hline 22. & 0.0 & 0.0 & $4.973 E-01$ \\
\hline
\end{tabular}

COEFFICIENT $2\left(P_{I}\right)$

FROM GROUP TRANSFER PROBABILITIES

$1.36 \mathrm{BE}-01$

$1.258 E-016.785 E-04$

$1.061 E-01-7.944 E-03 \quad 1.980 E-03$

9.999E-0 2-5.470E-03 $2.330 E-03 \quad 2.087 E-03$

1.313E-01-1.

$1.622 E-01-1.019 E-02 \quad 4.163 E-03 \quad 2.299 E-03 \quad 1.404 E-03$ 8.1 $05 E-04$

(1)

$1.212 E-01-3.286 E-02 \quad 5.393 E-03 \quad 3.264 E-03 \quad 1.990 E-03 \quad 1.086 E-03 \quad 6.636 E-04 \quad 3.829 E-04$

7.630E-02 7.260E-03-6.734E-04 1.077E-03 6.504E-04 3.966E-04 2.165E-04 1.322E-04 $7.632 \mathrm{E}-05$

$\begin{array}{lllllll} & 1.667 F-02-1.552 E-02 & 7.931 E-03 & 4.520 E-03 & 2.729 E-03 & 1.664 E-03 & 9.085 E-04\end{array}$ $49 E-043.202 E-04$

$\begin{array}{llllll} & \end{array}$ $1.002 E-01-1.08 B E-033.043 E-04$

$\begin{array}{llllll} & \end{array}$ 1.

$27.414[-03 \quad 5.541 E-03 \quad 4.332 E-03 \quad 2.615 E-03 \quad 1.480 E-03$

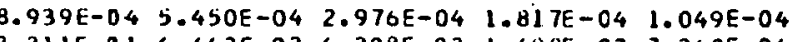

6.308E-03 1.68 8E-03 $7.360 E-04 \quad 5.500 E-04 \quad 4.300 E-04 \quad 2.596 E-04$ $05 \quad 5.411 E-05 \quad 2.95 .4 F-05 \quad 1.804 E-05$ 1. $041 E-05$

$\begin{array}{llllll} & \end{array}$ $3.178 E-011.289 E-014.542 E-072.648 E-07 \quad 1.445 E-07 \quad 8.828 E-08$ 5.095E-08

1. $24 E-07$ 4 . $2.809 E-01$ L. $175 E-019.404 E-03 \quad 3.021 E-041.784 E-061.473 E-07$ 3. $956 E-08$ 1.689E-09 $1.289 E-03$ 1. $008 E-08$ 6.083E-09 3.443E-C9 2.080E-09 1.208E-09 6.922E-10 4.228E-10 $2.439 E-10$

$2.503 E-011.352 E-011.828 E-021.323 E-03 \quad 4.249 E-05 \quad 2.510 E-072.079 E-085.565 E-09$ . $426 E-09$ 1. $012 E-091.418 E-098.556 E-104.844 E-10$ 2.925E-10 $1.784 E-10$ - $0.736 E-11$

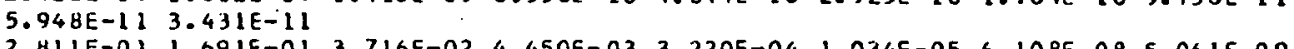

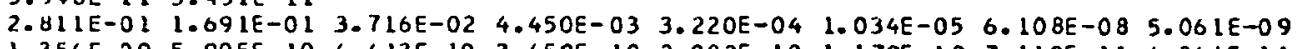
$1.354 E-095.905 E-10 \quad 4.413 E-10 \quad 3.450 E-10 \quad 2.082 E-10 \quad 1.179 E-10 \quad 7.119 E-114.342 E-11$ $2.370 E-11 \quad 1.448 E-11 \quad 6.841 E-12$

$2.550 E-01 \quad 1.351 E-01 \quad 2.686 E-02 \quad 5.226 E-03 \quad 6.259 E-04 \quad 4.529 E-05 \quad 1.455 E-06 \quad 8.591 E-09$ $7.119 E-10 \quad 1.905 E-10$ 8.306E-11 $6.208 E-11$ 4.853E-11 $2.929 E-111.658 E-119.784 E-12$ $5.100 E-12 \quad 1.342 \mathrm{E}-12 \quad 0.0 \quad 0.0$

$2.568 E-01 \quad 1.531 E-01 \quad 3.401 E-02 \quad 5.93 .3 E-03 \quad 1.165 E-03 \quad 1.396 E-04 \quad 1.010 E-05 \quad 3.244 E-07$ $1.916 E-091.587 E-104.248 E-11 \quad 1.852 E-11 \quad 1.383 E-11 \quad 1.082 E-11 \quad 5.390 E-12 \quad 2.078 E-12$ 0.0 $0.0 \quad 0.0$

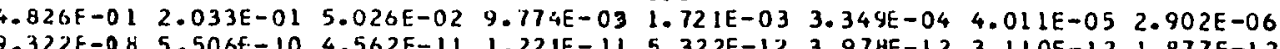
$\begin{array}{llll} & \end{array}$ 
CROSS SECTIONS AS READ FOR CONCRETE

\begin{tabular}{|c|c|c|}
\hline $\begin{array}{l}\text { GP } \\
1 \\
2 \\
3 \\
4 \\
5 \\
6 \\
7 \\
8\end{array}$ & $\begin{array}{l}\text { A85. } \times \text { SEC } \\
0.0 \\
0.0 \\
0.0 \\
0.0 \\
0.0 \\
0.0 \\
0.0 \\
0.0\end{array}$ & $\begin{array}{l}\text { NU\#FISS } \\
0.0 \\
0.0 \\
0.0 \\
0.0 \\
0.0 \\
0.0 \\
0.0 \\
0.0\end{array}$ \\
\hline 9 & 0.0 & 0.0 \\
\hline 10 & 0.0 & 0.0 \\
\hline 11 & 0.0 & 0.0 \\
\hline 12 & 0.0 & 0.0 \\
\hline 13 & 0.0 & 0.0 \\
\hline 14 & 0.0 & 0.0 \\
\hline 15 & 0.0 & 0.0 \\
\hline 16. & 0.0 & 0.0 . \\
\hline 17 & 0.0 & 0.0 \\
\hline 18 & 0.0 & 0.0 \\
\hline 19 & 0.0 & 0.0 \\
\hline 20 & 0.0 & 0.0 \\
\hline 21 & 0.0 & 0.0 \\
\hline 22 & 0.0 & 0.0 \\
\hline
\end{tabular}

\section{TOTAL}

$1.267 \mathrm{E}-01$

$1.274 E-01$

$1.195 \mathrm{E}-01$

$1.199 E-01$

$1.347 E-01$

$1.579 \varepsilon-01$

1. $991 E-01$

500E-O

$1.2106-01$

$1.688 \mathrm{~F}-01$

$2.198 E-01$

2.823E-01

3. $751 E-01$

4.231E-O1

4. $946 E-01$

4. $734 E-01$

4. $763 E-01$

$4.768 E-01$

4.777E-OI

4. $792 E-01$

4. $813 \mathrm{E}-0$

$4.813 \mathrm{E}-0$

4. $973 E-0$

COEFFICIENT $3\left(\mathrm{P}_{2}\right)$

FROM GROUP
$1.672 \mathrm{E}-0 \mathrm{I}$

. $531 E-013.147 E-O$

1.304E-0 4.882E-03 2.704E-03

1.192E-01 4.181E-03 $5.360 E-03 \quad 1.363 E-C 3$

1. $477 E-011.303 E-02$ 3.715E-03 $1.508 E-034.025 E-04$

.

$1.093 E-J 13.338 E-02 \quad 4.878 E-03$ 1.839E-03 4.85 LE-04-1.468E-04-2.898E-04-2.957E-04

8.577E-0.2-2.489E-03 5.208E-03 7.605E-04 2.437E-04 2.273E-05-6.965E-05-8.272E-05 $-7.373 E-05$

L.265E-J.1 $1.066 E-02 \quad 1.811 E-02 \quad 7.213 E-03 \quad 2.243 E-03 \quad 4.592 E-04-2.469 E-04-4.813 E-04$ $4.651 E-0.4-3.799 E-04$

$1.650 E-01$ 3.929E-02 1.738E-02 1.09 1E-02 3.420E-03-8.317E-05-1.078E-03-1.273E-03 $-1.109 E-03-8.856 E-04-6.490 E-04$

1.67JE-X1 5.82 2E-02 3.927E-03 5.436E-C4-1.042E-03-2. 449E-03-2.590E-03-2.199E-03 $1.733 E-33-1.217 E-03-8.834 E-04-6.046 E-04$

作 2.569E- $3-1.022 E-03-1.177 E-03-8.161 E-04-5.381 E-04$

$2.897 E-03-2.419 E-03-1.665 E-03$ $1.4 C 4 E-04$ (1) $3.341 E-E 15.968 E-03-1.516 E-02-5.301 F-04-8.694 E-05-3.235 E-05-1.735 E-05-1.394 E-05$ 作 -2.093E-2.6-1.733E-06-1. $176 \mathrm{E}-02-2.616 \mathrm{E}-03-7.994 \mathrm{E}-05-1.306 \mathrm{E}-05-4.858 \mathrm{E}-06-2.606 \mathrm{E}-06$ $-9.536 \mathrm{E}-3 \mathrm{~B}$

2.659E-01 6.136E-02-3.041E-02-7.573E-03-6.778E-04-2.030E-05-3.316E-06-1.233E-06 6.512E- $7-5.310 E-07-4.397 E-07-2.984 E-07-1.930 E-07-1.305 E-07-8.077 E-08-5.524 E-0 B$ $-3.743 E-38-2.420 E-0 B$

. $-5.123 E-97-2.748 E-07-2.207 E-07-1.827 E-07-1.240 E-07-8.021 E-08-5.423 E-08-3.689 E-08$ $-2.295 F-08-1.555 F-08-1.005 F-08$

$2.562 E-116.110 E-02-3.727 E-02-1.782 E-02-4.717 E-03-8.441 E-04-7.206 E-05-2.141 E-06$ $-3.497 E-97-1.300 E-07-6.973 E-08-5.600 E-C B-4.637 E-08-3.14 \pi-08-2.036 E-08-1.376 E-08$ $-9.361 E-39-5.825 E-09-3.947 E-09-2.552 E-C S$

$2.665 E-31 \quad 8.556 E-02-3.716 E-02-1.952 E-C 2-7.222 E-03-1.776 E-03-3.116 E-04-2.651 E-05$ $-7.872 E-37-1.286 E-07-4.779 E-D B-2.503 E-C B-2.059 E-08-1.705 E-08-1.157 E-08-7.483 E-09$ $-5.059 E-J 9-3.441 E-09-2.141 E-09-1.451 E-09-9.381 E-10$

$3.288 E-316.634 E-03-7.876 E-02-3.652 E-02-1.280 E-02-4.370 E-03-1.044 E-03-1.817 E-04$ $-1.543 E-55-4.583 E-07-7.483 E-08-2.732 E-C 8-1.492 E-08-1.198 E-08-9.924 E-09-6.735 E-09$ $-4.3566-09-2.946 E-09-2.003 E-09-1.247 E-09-8.447 E-10-5.461 E-10$ 
CROSS SECTIONS AS RFAD FOR CONCRETE

\begin{tabular}{|c|c|c|}
\hline $\begin{array}{l}6 P \\
1 \\
? \\
3 \\
4 \\
5 \\
6 \\
7 \\
8\end{array}$ & $\begin{array}{l}\text { ABS XSEC } \\
0.0 \\
0.0 \\
0.0 \\
0.0 \\
0.0 \\
0.0 \\
0.0 \\
0.0\end{array}$ & $\begin{array}{l}\text { NU.FIS } \\
0.0 \\
0.0 \\
0.0 \\
0.0 \\
0.0 \\
0.0 \\
0.0 \\
0.0\end{array}$ \\
\hline 9 & 0.0 & 0.0 \\
\hline 10 & 0.0 & 0.0 \\
\hline 11 & 0.0 & 0.0 \\
\hline 12 & 0.0 & 0.0 \\
\hline 13 & 0.0 & 0.0 \\
\hline 14 & 0.0 & 0.0 \\
\hline 15 & 0.0 & 0.0 \\
\hline 16 & 0.0 & 0.0 \\
\hline 17 & 0.0 & 0.0 \\
\hline 18 & 0.0 & 0.0 \\
\hline 19 & 0.0 & 0.0 \\
\hline 20 & 0.0 & 0.0 \\
\hline 21 & 0.0 & 0.0 \\
\hline 22 & 0.0 & 0.0 \\
\hline
\end{tabular}

\section{TOTAL}

1.26?E-01

$1.274 \mathrm{~F}-01$
$1.195 \mathrm{~F}-01$

$1.195 \mathrm{~F}=01$

$1.195 E-01$
$1.347 E-01$

$1.347 E-01$
$1.575 F-01$

$1.991 E-01$

$1.2105-0$

$1.688 \equiv-01$

$2.1985-01$

2. $823 E-01$

3. $751 E-01$

4. $231 E-01$

4. $946 \mathrm{E}-\mathrm{O}$

4. 734 E-OI

$4.763 F-01$

4. $.768 E-01$

4.77TE-O

4.792E-OL

4. 792E-OL

$4.813 F-01$

4. 97.3E-01
CCEFFICIENT $4 \quad\left(\mathrm{P}_{3}\right)$

FROM GROUP TRANSFER PROBABILITIES

\subsection{E-01}

$1.696 E-012.565 E-03$

$1.423 E-018.311 E-03 \quad 3.862 E-04$ $-1.901 E-04$ $1.331 E-03-8.398 \varepsilon-04$ $-2.654 E-03-1.736 E-03-1.060 E-03$ $-8.537 E-10$ $-8.293 E-11-5.067 E-11-2.973 E-11$
$1.257 E-019.960 E-03-6.172 E-04-9.273 E-C 4$

$1.437 E-01 \quad 1.578 E-02 \quad 6.987 E-04-1.248 E-03-1.576 E-03$

$1.458 F-011.787 F-021.211 E-03-1.312 E-03-1.573 E-03-1.300 E-03$

$1.524 E-01 \quad 1.500 E-02-1.906 E-03-2.22$ IE-03-2.858E-03-2.373E-03-1.689E-03

$7.447 E-02 \quad 1.032 E-02 \quad 5.283 E-04-2.034 E-03-2.368 E-03-1.872 E-03-1.365 E-03-9.001 E-04$

$6.675 E-02-2.849 E-03-1.261 E-03-3.653 E-04-6.339 E-04-5.839 E-04-4.201 E-04-2.953 E-04$

7.983E-02 2.043E-03 1.688E-02 7.146E-04-3.396E-03-3.560E-03-2.890E-03-1.948E-03

$8.848 E-021.460 E-031.748 E-03-4.414 E-03-8.747 E-03-8.081 E-03-6.108 E-03-4.310 E-03$

$6.049 E-02-1.113 E-02-1.704 E-02-1.643 E-02-1.478 E-02-1.094 E-02-7.232 E-03-4.774 E-03$ $-3.105 E-03-1.795 E-03-1.135 E-03-6.741 E-04$

$9.505 E-02-3.974 E-02-3.345 E-02-1.852 E-02-1.46 .1 E-02-1.182 E-02-7.550 E-03-4.489 E-03$ $-2.745 E-03-1.744 E-03-9.728 E-04-6.020 E-04-2.514 E-04$

$9.501 E-02-8.674 E-02-1.854 E-02-5.423 E-C 3-2.437 E-03-1.836 E-03-1.443 E-03-8.786 E-04$ $-5.014 E-04-3.044 E-04-1.863 E-04-1.02 \mathrm{IE}-04-6.251 E-05-3.615 E-05$

$1.943 E-01-8.075 E-02-1.276 E-03-1.075 E-C 4-2.884 E-05-1.258 E-05-9.407 E-06-7.356 E-06$ $-4.441 E-06-2.515 E-06-1.519 E-06-9.262 E-07-5.057 E-07-3.089 E-07-1.783 E-07$

$1.941 \mathrm{E}-01-1.599 \mathrm{E}-01-1.422 \mathrm{E}-02-9.407 E-05-7.817 \mathrm{E}-06-2.093 E-06-9.126 E-07-6.820 \mathrm{E}-07$ $-5.333 F-07-3.219 E-07-1.822 E-07-1.10$ LE-07-6. 7LOE-08-3.663E-08-2.237E-08-1.241E-08 $2.219 E-01-1.269 E-01-2.880 E-02-1.036 E-03-6.240 E-06-5.173 E-07-1.385 E-07-6.036 E-08$ $-4.511 E-08-3.527 E-08-2.129 E-08-1.205 E-C 8-7.279 E-09-4.438 E-09-2.422 E-09-1.480 E-09$

$2.298 E-01-1.067 E-01-5.016 E-02-4.456 E-03-1.470 E-04-8.782 E-07-7.278 E-08-1.948 E-08$ $-8.492 E-09-6.344 E-09-4.962 E-09-2.994 E-09-1.696 E-09-1.024 E-09-6.243 E-10-3.408 E-10$ $-2.082 \mathrm{E}-1.0-1.201 \mathrm{E}-10$

$2.219 E-01-1.335 E-01-9.344 E-02-1.439 E-C 2-1.112 E-03-3.612 E-05-2.13 \mathrm{dE}-07-1.771 E-08$ $-4.740 E-09-2.067 E-09-1.545 E-09-1.207 E-05-7.289 E-10-4.126 E-10-2.492 E-10-1.519 E-10$

$2.299 E-01-1.070 E-01-7.145 E-02-1.075 E-02-2.141 E-03-1.579 E-04-5.088 E-06-3.007 E-08$ $-2.492 E-09-6.668 E-10-2.907 E-10-2.173 E-10-1.698 E-10-1.025 E-10-5.804 E-11-3.305 E-11$ $-2.137 E-11-1.167 E-11-6.596 E-12-2.793 E-12$

$2.300 F-01-1.000 F-01-8.402 E-02-1.911 F-02-3.957 E-03-40.844 E-04-3.529 F-05-1.135 E-06$ $-6.105 E-09-5.556 E-10-1.487 E-10-6.482 E-11-4.842 E-11-3.788 E-11-2.280 E-11-1.294 E-11$ $7.254 E-12-2.999 E-12-5.326 E-130.0 \quad 0.0$

$5.547 F-02-2.302 E-01-1.301 E-01-3.108 E-02-5.858 E-03-1.161 E-03-1.400 E-04-1.015 E-05$ $-3.263 E-07-1.927 F-09-1.597 E-10-4.273 E-11-1.863 E-11-1.392 E-11-1.08 d E-11-6.570 E-12$
$-3.719 E-12-2.246 F-12-1.369 E-120.0$ 


\begin{tabular}{|c|c|c|c|c|c|c|c|c|c|c|c|c|c|c|}
\hline GROU & SIGT & SIGST & $\begin{array}{l}55 \text { SEC } \\
\text { PNUP }\end{array}$ & $\begin{array}{l}\text { IONS FOR } \\
\text { PNABS }\end{array}$ & $\begin{array}{c}\text { CONCRE } \\
\text { GAMGEN }\end{array}$ & $\begin{array}{l}\text { ETE } \\
\text { NU*FIS }\end{array}$ & DOHNSCAT & TTER PRUB & BABILIYY & & & & & \\
\hline & $1.26 \sqrt{E}-01$ & $1.12 \pi-01$ & 0.0 & 0.8894 & 0.0 & 0.0 & $\begin{array}{c}0.5205 \\
0.0099 \\
0.0000\end{array}$ & $\begin{array}{c}0.0694 \\
0.0435 \\
0.0000\end{array}$ & $\begin{array}{l}0.0209 \\
0.0518 \\
0.000 C\end{array}$ & $\begin{array}{c}0.0578 \\
0.0365 \\
0.0000\end{array}$ & $\begin{array}{c}0.0523 \\
0.0242 \\
0.0000\end{array}$ & $\begin{array}{c}0.0247 \\
0.0025 \\
0.0000\end{array}$ & $\begin{array}{c}0.0483 \\
0.0000\end{array}$ & $\begin{array}{l}0.0358 \\
0.0000\end{array}$ \\
\hline 2 & $1.274 E-01$ & $1.079 E-01$ & 0.0 & 0.8467 & 0.0 & 0.0 & $\begin{array}{r}0.5064 \\
0.0267 \\
0.0000\end{array}$ & $\begin{array}{c}0.1658 \\
0.0416 \\
0.0000\end{array}$ & $\begin{array}{c}0.0320 \\
0.0313 \\
0.00 .0 \mathrm{C}\end{array}$ & $\begin{array}{c}0.0274 \\
0.0173 \\
0.0000\end{array}$ & $\begin{array}{r}0.0474 \\
0.0019 \\
0.0000\end{array}$ & $\begin{array}{l}0.0692 \\
0.0000\end{array}$ & $\begin{array}{c}0.0260 \\
0.0000\end{array}$ & $\begin{array}{r}0.0070 \\
0.0000\end{array}$ \\
\hline 3 & $1.195 F-01$ & $1.035 E-01$ & 0.0 & 0.8661 & 0.0 & 0.0 & $\begin{array}{c}0.4518 \\
0.0695 \\
0.0000\end{array}$ & $\begin{array}{l}0.2109 \\
0.0373 \\
0.0000\end{array}$ & $\begin{array}{c}0.0347 \\
0.0177 \\
0.0360\end{array}$ & $\begin{array}{c}0.0265 \\
0.0020 \\
0.0000\end{array}$ & $\begin{array}{l}0.0422 \\
0.00 \mathrm{co}\end{array}$ & $\begin{array}{c}0.0416 \\
0.0000\end{array}$ & $\begin{array}{c}0.0098 \\
0.0000\end{array}$ & $\begin{array}{c}0.0559 \\
0.0000\end{array}$ \\
\hline 4 & $1.1998-01$ & $1.086 E-01$ & 0.0 & 0.9062 & 0.0 & 0.0 & $\begin{array}{c}0.4323 \\
0.0449 \\
0.0000\end{array}$ & $\begin{array}{c}0.2700 \\
0.0327 \\
0.0000\end{array}$ & $\begin{array}{r}0.0579 \\
0.0050 \\
0.000 \mathrm{C}\end{array}$ & $\begin{array}{c}0.0420 \\
0.0001\end{array}$ & $\begin{array}{c}0.0262 \\
0.0000\end{array}$ & $\begin{array}{c}0.0067 \\
0.0000\end{array}$ & $\begin{array}{c}0.0297 \\
0.0000\end{array}$ & $\begin{array}{c}0.0524 \\
0.0000\end{array}$ \\
\hline 5 & $1.34 \pi-01$ & $1.297 E-01$ & 0.0 & 0.9628 & 0.0 & 0.0 & $\begin{array}{c}0.5257 \\
0.0247 \\
0.0000\end{array}$ & $\begin{array}{c}0.2028 \\
0.0048 \\
0.0000\end{array}$ & $\begin{array}{c}0.0957 \\
0.0001\end{array}$ & $\begin{array}{c}0.0 \geq 15 \\
0.0000\end{array}$ & $\begin{array}{c}0.0057 \\
0.0000\end{array}$ & $\begin{array}{l}0.0297 \\
0.0000\end{array}$ & $\begin{array}{c}0.0443 \\
0.0600\end{array}$ & $\begin{array}{r}0.0349 \\
c .0000\end{array}$ \\
\hline 6 & $1.579 F-01$ & $1.531 E=01$ & 0.0 & 0.9693 & 0.0 & 0.0 & $\begin{array}{c}0.5110 \\
0.0047 \\
0.0000\end{array}$ & $\begin{array}{r}0.2668 \\
0.000 \mathrm{~L}\end{array}$ & $\begin{array}{r}0.0534 \\
0.0000\end{array}$ & $\begin{array}{c}0.0142 \\
0.0000\end{array}$ & $\begin{array}{c}0.0471 \\
0.0000\end{array}$ & $\begin{array}{c}0.0445 \\
0.0000\end{array}$ & $\begin{array}{c}0.0365 \\
0.0000\end{array}$ & $\begin{array}{c}0.0218 \\
0.0000\end{array}$ \\
\hline 1 & $1.991 E-01$ & $1.975 E-01$ & 0.0 & 0.49 .18 & 0.0 & 0.0 & $\begin{array}{c}0.0053 \\
0.0001\end{array}$ & $\begin{array}{l}0.2207 \\
0.0000\end{array}$ & $\begin{array}{l}0.0115 \\
0.0000\end{array}$ & $\begin{array}{c}0.0362 \\
0.0000\end{array}$ & $\begin{array}{l}0.0644 \\
0.0000\end{array}$ & $\begin{array}{c}0.0325 \\
0.0000\end{array}$ & $\begin{array}{c}0.0244 \\
0.0000\end{array}$ & $\begin{array}{r}0.0045 \\
0.0000\end{array}$ \\
\hline B & $1.500 E-01$ & $1.495 E-01$ & 0.0 & C. 9964 & 0.0 & 0.0 & $\begin{array}{c}0.4717 \\
0.0000\end{array}$ & $\begin{array}{c}0.1254 \\
0.0000\end{array}$ & $\begin{array}{l}0.1934 \\
0.0000\end{array}$ & $\begin{array}{c}0.0707 \\
0.0000\end{array}$ & $\begin{array}{c}0.0943 \\
0.0000\end{array}$ & $\begin{array}{c}0.0367 \\
0.0000\end{array}$ & $\begin{array}{c}0.0076 \\
0.0000\end{array}$ & 0.0002 \\
\hline 9 & $1.216 E-01$ & $1.213 E-01$ & 0.0 & 0.9973 & 0.0 & 0.0 & $\begin{array}{l}0.2559 \\
0.0000\end{array}$ & $\begin{array}{l}0.4553 \\
0.0000\end{array}$ & $\begin{array}{c}0.1163 \\
0.000 \mathrm{C}\end{array}$ & $\begin{array}{l}0.0761 \\
0.0000\end{array}$ & $\begin{array}{l}0.0847 \\
0.0000\end{array}$ & $\begin{array}{l}0.0114 \\
0.0000\end{array}$ & 0.0003 & 0.0000 \\
\hline 10 & $1.688 F-01$ & $1.68 \Delta E-01$ & 0.0 & 0.9988 & 0.0 & 0.0 & $\begin{array}{c}0.5346 \\
0.0000\end{array}$ & $\begin{array}{c}0.3242 \\
0.0000\end{array}$ & $\begin{array}{c}0.0617 \\
0.000 \mathrm{C}\end{array}$ & $\begin{array}{c}0.0672 \\
0.0000\end{array}$ & $\begin{array}{c}0.0120 \\
0.0000\end{array}$ & 0.0002 & 0.0000 & 0.0000 \\
\hline 11 & $2.198 F-01$ & $2.197 E-01$ & 0.0 & 0.9995 & 0.0 & 0.0 & $\begin{array}{c}0.7004 \\
0.0000\end{array}$ & $\begin{array}{c}0.2231 \\
0.0000\end{array}$ & $\begin{array}{l}0.0628 \\
0.000 \mathrm{C}\end{array}$ & $\begin{array}{l}0.0134 \\
c .0000\end{array}$ & 0.0003 & 0.0001 & 0.0000 & 0.0000 \\
\hline 12 & $2.823 E-01$ & $2.822 F-01$ & 0.0 & 0.9997 & 0.0 & 0.0 & $\begin{array}{c}0.7651 \\
0.0000\end{array}$ & $\begin{array}{l}0.2058 \\
0.0000\end{array}$ & $\begin{array}{l}0.0283 \\
0.0000\end{array}$ & 0.0007 & 0.0001 & 0.0000 & 0.0000 & 0.0000 \\
\hline 13 & $3.751 E-01$ & $75 \alpha E-01$ & 0.0 & 0.9998 & 0.0 & 0.0 & $\begin{array}{c}0.8386 \\
0.0000\end{array}$ & $\begin{array}{r}0.1574 \\
0.0000\end{array}$ & $0 . \simeq 033$ & 0.0006 & 0.0001 & 0.0000 & 0.0000 & 0.0000 \\
\hline 14 & $231 F-01$ & $229 E-0$ & 0.0 & 994 & 0.0 & 0.0 & $\begin{array}{c}0.8661 \\
0.0000\end{array}$ & 0.1133 & 0.0170 & 0.0025 & 0.0006 & 0.0003 & 0.0001 & 0.0000 \\
\hline 15 & $4.946 E-01$ & $4.936 E-01$ & 0.0 & 0.9979 & 0.5 & 0.0 & 0.7655 & 0.1984 & 0.11257 & 0.0065 & 0.0027 & 0.0007 & 0.0003 & 0.0001 \\
\hline $\begin{array}{l}16 \\
17 \\
18 \\
19 \\
20 \\
21 \\
22\end{array}$ & $\begin{array}{l}4.734 E-01 \\
4.763 E-01 \\
4.763 E-01 \\
4.777 E-01 \\
4.792 E-01 \\
4.813 E-01 \\
4.973 E-01\end{array}$ & $\begin{array}{l}4.731 E-01 \\
4.757 E-01 \\
4.759 E-01 \\
4.761 E-01 \\
4.765 E-01 \\
4.767 E-01 \\
4.744 E-01\end{array}$ & $\begin{array}{l}0.0 \\
0.0 \\
0.0 \\
0.0 \\
0.0 \\
0.0 \\
0.0\end{array}$ & $\begin{array}{l}0.9995 \\
0.9989 \\
0.9931 \\
0.9967 \\
0.9942 \\
0.9905 \\
0.9541\end{array}$ & $\begin{array}{l}0.0 \\
0.0 \\
0.0 \\
0.0 \\
0.0 \\
0.0 \\
0.0\end{array}$ & $\begin{array}{l}0.0 \\
0.0 \\
3.0 \\
0.0 \\
0.0 \\
0.0 \\
0.0\end{array}$ & $\begin{array}{l}0.7548 \\
0.6992 \\
0.6612 \\
0.6997 \\
0.6622 \\
0.6627 \\
1.0000\end{array}$ & $\begin{array}{l}0.1831 \\
0.2047 \\
0.2559 \\
0.2044 \\
0.2317 \\
0.3373\end{array}$ & $\begin{array}{l}0.0392 \\
0.0686 \\
0.0524 \\
0.0606 \\
0.1062\end{array}$ & $\begin{array}{l}0.0163 \\
0.0174 \\
0.0193 \\
0.0353\end{array}$ & $\begin{array}{l}0.0041 \\
0.0064 \\
0.0112\end{array}$ & $\begin{array}{l}0.0015 \\
0.0037\end{array}$ & 0.0009 & \\
\hline
\end{tabular}


ORNL/TM-5414

UC-79d - LMFBR

Physics

\section{INTERNAL DISTRIBUTION}

1-3. L. S. Abbott

4. R. G. Alsmiller, Jr.

5. D. E. Bartine

6. V. C. Baker

7. J. A. Bucholz

8. R. L. Childs

9. C. E. Clifford

10. S. N. Cramer

11. G. W. Cunningham

12. M. B. Emmett

13. W. W. Engle, Jr.

14. G. F. Flanagan

15. W. E. Ford, III

16. T. B. Fowler

17. S. K. Fraley

18. T. A. Gabriel

19. H. Goldstein (Consultant)

201 N. M. Greene

21. W. O. Harms

22. 0. W. Hermann

23-27. T. J. Hoffman

28. J. R. Knight

29. R. A. Lillie

30. J. L. Lucius

31. R. E. Maerker

32. J. W. McAdoo

33. F. C. Maienschein

34. G. W. Morrison

35. F. R. Mynatt

36. B. Nakhai

37. J. V. Pace, III

38. L. M. Petrie

39. H. Postma

40. W. A. Rhoades
41. J. C. Robinson

42. R. T. Santoro

43. D. L. Selby

44. D. B. Simpson

45. C. 0. Slater

46-50. P. N. Stevens

51-65. J. S. Tang

66. J. T. Thomas

67. M. L. Tobias

68. E. T. Tomlinson

69. D. B. Trauger

70. D. K. Trubey

71. K. R. Turnbul1

72. D. R. Vondy

73. C. R. Weisbin

74. R. M. Westfall

75. J. E. White

76. G. E. Whitesides

77. L. R. Williams

78. M. L. Williams

79. A. Zucker

80. P. F. Fox (Consultant)

81. W. W. Havens (Consultant)

82. A. F. Henry (Consultant)

83. R. E. Uhrig (Consultant)

84-85. Central Research Library

86. Y-12 Document Reference Section

87-88. Laboratory Records Department

89. Laboratory Records, ORNL RC

90-95. Radiation Shielding Information Center

\section{EXTERNAL DISTRIBUTION}

96-97. Director, Division of Reactor Development and Demonstration, Energy Research and Development Administration, Washington, DC 20545.

98. Research and Technical Support Division, Energy Research and Development Administration, Oak Ridge, TN 37830.

99. Director, Reactor Division, Energy Research and Development Administration, Oak Ridge, TN 37830.

100-305. For distribution as shown in TID-4500, Distribution Category UC-79d, Liquid Metal Fast Breeder Reactor Physirs. 